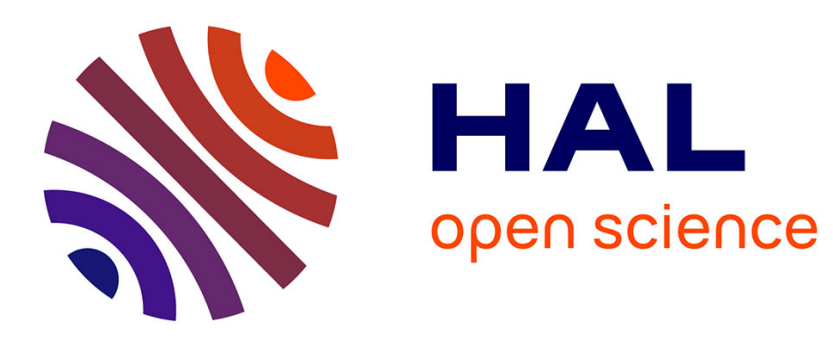

\title{
La physique nucléaire en astrophysique
}

\author{
M. Arnould, Mickaël Samyn
}

\section{To cite this version:}

M. Arnould, Mickaël Samyn. La physique nucléaire en astrophysique. École thématique. Ecole Joliot Curie "Astrophysique nucléaire: du microcosme au macrocosme astrophysique", Spa, (Belgique), du 10-16 septembre 2000 : 19ème session, 2000. cel-00653818

\section{HAL Id: cel-00653818 https://cel.hal.science/cel-00653818}

Submitted on 20 Dec 2011

HAL is a multi-disciplinary open access archive for the deposit and dissemination of scientific research documents, whether they are published or not. The documents may come from teaching and research institutions in France or abroad, or from public or private research centers.
L'archive ouverte pluridisciplinaire HAL, est destinée au dépôt et à la diffusion de documents scientifiques de niveau recherche, publiés ou non, émanant des établissements d'enseignement et de recherche français ou étrangers, des laboratoires publics ou privés. 


\title{
LA PHYSIQUE NUCLEAIRE EN ASTROPHYSIQUE
}

\author{
M. Arnould et M. Samyn \\ Institut d'Astronomie et d'Astrophysique, \\ Université Libre de Bruxelles, Campus Plaine, CP 226, \\ B-1050 Bruxelles, Belgique
}

Résumé

L'astrophysique nucléaire est sans nul doute l'une des branches les plus largement interdisciplinaires de la science et est intimement connectée à une très grande variété d'autres domaines de recherche. Ce cours se limite à une brève présentation des relations privilégiées qui se sont établies entre l'astrophysique et la physique nucléaire.

Nous montrons que la physique nucléaire et l'astrophysique apportent une contribution importante à la compréhension d'un ensemble rapidement croissant d'observations relatives à 1'Univers et à ses constituants les plus divers, allant de grains sub-millimétriques dans les météorites à des galaxies extrêmement lointaines. Au travers de divers exemples, nous illustrons également le fait que l'astrophysique pousse très souvent la physique nucléaire dans ses 'derniers retranchements' et à explorer des 'terres inconnues' situées bien loin de la vallée de stabilité nucléaire. Bien que des progrès considérables ont été réalisés dans cette exploration, nous mettons en évidence que bien du travail reste à faire et qu'une meilleure compréhension mutuelle entre physiciens nucléaires et astrophysiciens ne pourrait manquer d'être la source de nouvelles et exaltantes aventures scientifiques.

\begin{abstract}
There is no doubt that nuclear astrophysics is one of the widest interdisciplinary fields of modern science through its many tight connections with a rich variety of other scientific disciplines. These lectures are only concerned with a brief presentation of the privileged relationship between astrophysics and nuclear physics.

We show that nuclear physics and astrophysics both bring an important share to our understanding of an ever-growing body of observations of the Universe and of its large diversity of constituants, ranging from sub-millimeter grains in meteorites to very distant galaxies. Various examples help us to illustrate that astrophysics very often pushes nuclear physics 'to the wall' and to explore the terra incognita located far away from the valley of nuclear stability. Even if substantial progress has been made in this exploration, much obviously remains to be done. Clearly, a better understanding between nuclear physicists and astrophysicists would succeed in promoting further scientific excitements.
\end{abstract}

\section{Introduction}

L'astrophysique, au travers de l'union de l'astronomie et la physique, tente de déchiffrer la macro-structure de l'Univers et de ses multiples composantes. Pour atteindre ce but, les lois physiques dégagées dans le laboratoire terrestre sont systématiquement appliquées à l'énorme diversité des situations cosmiques dans le cadre d'une recherche très largement interdisciplinaire. En particulier, la cosmologie, chaque branche de l'astronomie, l'astronautique, la physique des particules élémentaires, des noyaux, des atomes et des molécules, de mêrne que la géo- et la cosmochimie participent à cette aventure scientifique.

Très souvent, les objets cosmiques possèdent des propriétés mettant clairement en évidence la signature de la micro-physique des particules élémentaires ou des noyaux atomiques. Ce qui suit traite de la relation privilégiée entre la physique nucléaire 
et l'astrophysique ayant donné naissance à une discipline tout naturellement appelée 'astrophysique nucléaire'. Son but premier est de fournir une explication à l'énorme énergie libérée par les objets cosmiques, et en particulier par les étoiles, ainsi que de construire une image cohérente des variations spatiales et temporelles de l'abondance des nucléides dans l'Univers et dans ses divers constituants.

L'hypothèse que la production d'énergie dans le Soleil et dans les étoiles en général résulte de transmutations nucléaires a apparemment été formulée pour la première fois et peu de temps après les premières mesures de masses atomiques par Russell [1], suivi de près par Perrin [2]. La découverte du neutron en 1932 et une meilleure compréhension du noyau atomique qui en a résulté ont permis à Gamow, von Weizsäcker, Bethe et d'autres de développer cette idée sur une base plus quantitative. C'est ainsi que la source de l'énergie solaire fut identifiée comme étant les 'chaînes proton-proton', dont le résultat global est la transformation de 4 protons en un noyau de ${ }^{4} \mathrm{He}$, accompagnée de la production d'environ $6 \mathrm{MeV}$ par protonł. Une multitude de travaux ultérieurs ont confirmé de façon éclatante la validité de ces idées.

Le rôle central joué par les réactions nucléaires dans 'l'alchimie' de l'Univers commença également à être reconnu, conduisant au développement d'un chapitre essentiel de l'astrophysique nucléaire appelé 'théorie de la nucléosynthèse'. Au fil d'observations de plus en plus nombreuses, et en particulier de celle de l'élément instable technetium (dont aucun isotope ne possède une vie moyenne supérieure à quelques millions d'années) à la surface de certaines étoiles Géantes Rouges de type S [4], il apparut que les étoiles constituent bien des agents nucléosynthétiques majeurs, une idée avancée par Hoyle dès 1946 [3]. A la fin des années 50 , les modèles de nucléosynthèse stellaire furent reconnus capables d'expliquer l'origine de la grande majorité des nucléides de nombre de masse $A \geq 12$ existant à l'état naturel ([5]).§ Une étape très importante dans le développement de ces idées fut l'identification $[6,7]$ de la transformation nucléaire appelée ' $3 \alpha$ ' permettant de franchir la lacune en nucléides stables à $A=8$ par la transmutation de 3 particules $\alpha$ en un noyau de ${ }^{12} \mathrm{C}$ dans laquelle le noyau non lié ${ }^{8} \mathrm{Be}$ joue un rôle fondamental. Sur base des abondances stellaires relatives de ${ }^{4} \mathrm{He},{ }^{12} \mathrm{C}$ et ${ }^{16} \mathrm{O}$, Hoyle fut même capable de prédire l'existence d'un état résonnant $0^{+}$de ${ }^{12} \mathrm{C}$ à $7.7 \mathrm{MeV}$ qui fut découvert expérimentalement peu de temps après ([8]). En dépit de ces multiples succès, l'abondance naturelle de certains nucléides, et en particulier des nucléides légers $\mathrm{D},{ }^{3} \mathrm{He},{ }^{4} \mathrm{He},{ }^{6} \mathrm{Li},{ }^{7} \mathrm{Li},{ }^{9} \mathrm{Be},{ }^{10} \mathrm{~B}$ et ${ }^{11} \mathrm{~B}$ restait pourtant difficile à expliquer\|.

Depuis cette époque 'héroïque', l'astrophysique nucléaire a connu des progrès aussi nombreux que spectaculaires. Ils sont dus à de multiples avancées dans un grand nombre de domaines, comme la physique nucléaire expérimentale et théorique, les observations astronomiques à partir du sol et de l'espace, ainsi que la modélisation astrophysique. En fait, l'astrophysique nucléaire n'a cessé tout à la fois d'être mise en question et de trouver ses sources d'inspiration par de nouvelles découvertes, dont certaines ont marqué l'histoire des sciences en général et de l'astrophysique en particulier. N'en citons que quelques-unes: (1) la découverte en 1965 du fond micro-onde à $3 \mathrm{~K}$, qui est l'un des 'pilliers' du modèle du Big Bang, accompagné d'une nucléosynthèse 'primordiale' responsable de la production de quelques-uns des nucléides légers mentionnés ci-dessus;

$\ddagger$ Environ $2 \times 10^{19} \mathrm{~kg}$ ou $10^{-11} M_{\odot}$ de protons brûlent actuellement par an dans le Soleil, dont la masse totale $M_{\odot} \approx 2 \times 10^{30} \mathrm{~kg}$

$\S$ Ici et dans le reste du texte, la notation ([n]) signifie (voir par ex. [n] et les références citées dans ce travail) II La production de $\mathrm{H}$ sort du cadre normal de la théorie de la nucléosynthše, et constitue en fait un chapitre de la théorie de la 'baryogenèse' 
(2) la détection à la fin des années 60 de neutrinos émis par le Soleil, et fournissant la première 'vision' de l'intérieur d'une étoile. Avec le temps. il est apparu de plus en plus évident que le flux de neutrinos observé était inférieur à celui calculé. Le fameux 'problème des neutrinos solaires' était né, impliquant une connaissance incomplète de la structure et de l'évolution du Soleil (et sans doute des étoiles en général), des réactions nucléaires dans les intérieurs stellaires, de la physique des neutrinos dans la matière stellaire ou dans le vide, ou toute combinaison de ces divers problèmes;

(3) la découverte dans une minuscule fraction du matériau météoritique d'un ensemble d'éléments chimiques dont la composition isotopique diffère de celle du gros de la matière solaire. Certaines de ces 'anomalies isotopiques' sont même dues à la désintégration in situ de radionucléides dont la demi-vie $\left(t_{1 / 2}\right)$ se situe entre $10^{5}$ et $10^{8}$ ans environ. Un remarquable exemple de ce type est fourni par ${ }^{26} \mathrm{Al}\left(t_{1 / 2}=7.4 \times 10^{5}\right.$ ans $)$. Qui plus est, la trace de radionucléides dont la demi-vie est aussi courte que 2.6 ans $\left({ }^{22} \mathrm{Ne}\right)$ ou 60 ans $\left({ }^{44} \mathrm{Ti}\right)$ a été identifiée dans certaines météorites;

(4) la découverte dans le milieu interstellaire d'une raie $\gamma$ émise lors de la désexcitation du

${ }^{26} \mathrm{Mg}$ produit par la désintégration $\beta$ de ${ }^{26} \mathrm{Al}$. Cette détection a conduit au développement rapide d'une nouvelle astronomie, celle des raies $\gamma$ nucléaires;

(5) l'explosion en février 1987 de la supernova SN1987A dans le Grand Nuage de Magellan a constitué une véritable pierre angulaire dans de nombreux domaines de l'astrophysique. La détection de quelques neutrinos émis par cette explosion a permis d'ouvrir le nouveau chapitre de l'astrophysique des neutrinos non-solaires. De nouvelles et importantes observations sont venues enrichir l'astrophysique des raies $\gamma$ nucléaires, ainsi que l'astrophysique nucléaire, et tout particulièrement la théorie de la nucléosynthèse explosive (voir B. Cordier, ce volume).

Afin d'être en mesure de relever le défi permanent lancé par de nouvelles et nombreuses données observationnelles, les concepts et les modèles de l'astrophysique nucléaire doivent reposer sur des bases de plus en plus solides. Ce but ne peut être atteint qu'au travers d'une meilleure compréhension des nombreux processus nucléaires susceptibles de se développer dans les environnements astrophysiques, complétée par une meilleure modélisation de ceuxci. Dans ce cadre, l'acquisition de nouvelles données nucléaires est évidemment indispensable, ce qui est manifestement plus facile à dire qu'à faire ! En effet, il est généralement très difficile, sinon impossible, de simuler en laboratoire le comportement d'un noyau dans les conditions astrophysiques, ou même de produire des noyaux susceptibles d'être impliqués dans ces conditions. C'est pourquoi il ne suffit en général pas de développer de nouvelles techniques expérimentales, une approche théorique de la description des noyaux s'avérant constituer un complément indispensable.

Les questions de physique nucléaire et d'astrophysique intéressant l'astrophysique nucléaire sont d'une telle diversité qu'il n'est pas possible de les passer en revue de façon exhaustive. Nous nous limiterons donc dans ces leçons à une discussion de quelques aspects seulement des énormes efforts qui ont été consentis en vue de déchiffrer les empreintes du nucléaire dans l'Univers. Le lecteur peut se référer à quelques manuels $([9,10,11,12])$ ou à une revue récente [13] pour obtenir des informations complémentaires.

\section{Quelques observations astronomiques pertinentes}

Le fondement observationnel de l'astrophysique nucléaire, et plus particulièrement de la théorie de la nucléosynthèse, repose largement sur la détermination de l'abondance des éléments et de leurs isotopes dans la plus grande variété possible d'objets cosmiques, ainsi 
que sur l'étude de la plus large variété possible d'observables caractérisant ces objets. Ces observations concernent presqu'exclusivement l'étude détaillée de la lumière en provenance de notre Galaxie (son milieu interstellaire aussi bien que les étoiles de divers types ayant ou non subi une explosion), aussi bien que d'autres galaxies, parmi lesquelles certaines peuplaient déjà l'Univers dans sa prime jeunesse. Des avancées récentes en astronomie optique, complétées par le développement fulgurant de 'nouvelles' astronomies (en particulier infra-rouge, $\mathrm{UV}, \mathrm{x}$ et $\gamma$ ) nous permettent aujourd'hui de voir l'Univers à toutes les longueurs d'onde, depuis les fréquences radio jusqu'à des énergies $\gamma$ de l'ordre du $\mathrm{TeV}$ ou du PeV. Dans bien des cas, les énormes progrès réalisés dans cette observation de l'Univers découlent directement de ceux dont bénéficient les technologies spatiales ([14]). Les propriétés de la lumière observée sont souvent associées à la température des zones émettrices, la situation la plus remarquable de ce type étant la radiation du corps noir. Des sources non-thermiques sont également identifiées, et se caractérisent généralement par des photons de haute énergie (en particulier dans les domaines $\mathrm{x}$ et $\gamma$ ). La radiation Bremsstrahlung ou synchrotron ainsi que celle provenant de désintégrations radioactives sont de bons exemples d'émission non-thermique.

L'observation du rayonnement électromagnétique à différentes longueurs d'onde est complétée par l'analyse détaillée de l'infime quantité de matière de l'Univers accessible à l'homme. La presque totalité de cette matière se trouve dans le système solaire lui-même. Le reste vient du rayonnement cosmique (extra-)galactique. Les observations des neutrinos solaires ou non-solaires représentent également des étapes importantes dans I'histoire de l'astrophysique nucléaire, ainsi que dans bien d'autres domaines de l'astrophysique.

Il est impossible de passer en revue la myriade de données d'observations pouvant intéresser l'astrophysique nucléaire. Nous nous limitons donc à quelques exemples illustrant l'importance de déchiffrer les messages électromagnétiques venus du ciel.

\subsection{Le diagramme de Hertzsprung-Russell (DHR)}

Le DHR fournit la représentation d'un échantillon d'étoiles dans un plan (couleur, brillance de surface) ou dans tout plan équivalent, où la couleur est remplacée par un 'indice de couleur', 'type spectral' ou 'température effective', et la brillance de surface ('luminosité') par une échelle de 'magnitudes' (voir [15] pour la définition de ces quantités et les diverses relations entre elles). L'adoption des échelles de couleur et de magnitudes conduit à une représentation 'couleur-magnitude' du DHR, dont deux exemples somt fournis aux Figs. 1 et 2 .

Le DHR est généralement considéré comme la 'pierre de Rosette' de l'évolution stellaire en raison du rôle central qu'il a joué dans le développement de cette branche de l'astrophysique. La caractéristique la plus remarquable de ce diagramme est sans conteste l'existence de concentrations d'étoiles d'un échantillon donné le long de lignes de corrélation, dont la plus spectaculaire est la 'séquence principale'. 'Il faut également remarquer que la population relative des différentes lignes, ou même des différentes portions d'une même ligne, dépend de l'échantillon considéré. Par exemple, la branche des 'géantes rouges (RG)' ou des 'géantes rouges asymptotiques (AGB)' est pratiquement absente du DHR d'amas 'ouverts' (voir Fig. 2), alors que la séquence principale est bien développée. La situation est très différente dans les amas globulaires (Fig. 2). La théorie de la structure et de l'évolution stellaire est à présent en mesure d'expliquer ces caractéristiques spécifiques du DHR. Sans entrer dans les détails, soulignons quelques résultats d'un grand intérêt: (1) il est possible de démontrer formellement que l'existence de lignes de corrélation (au lieu de 

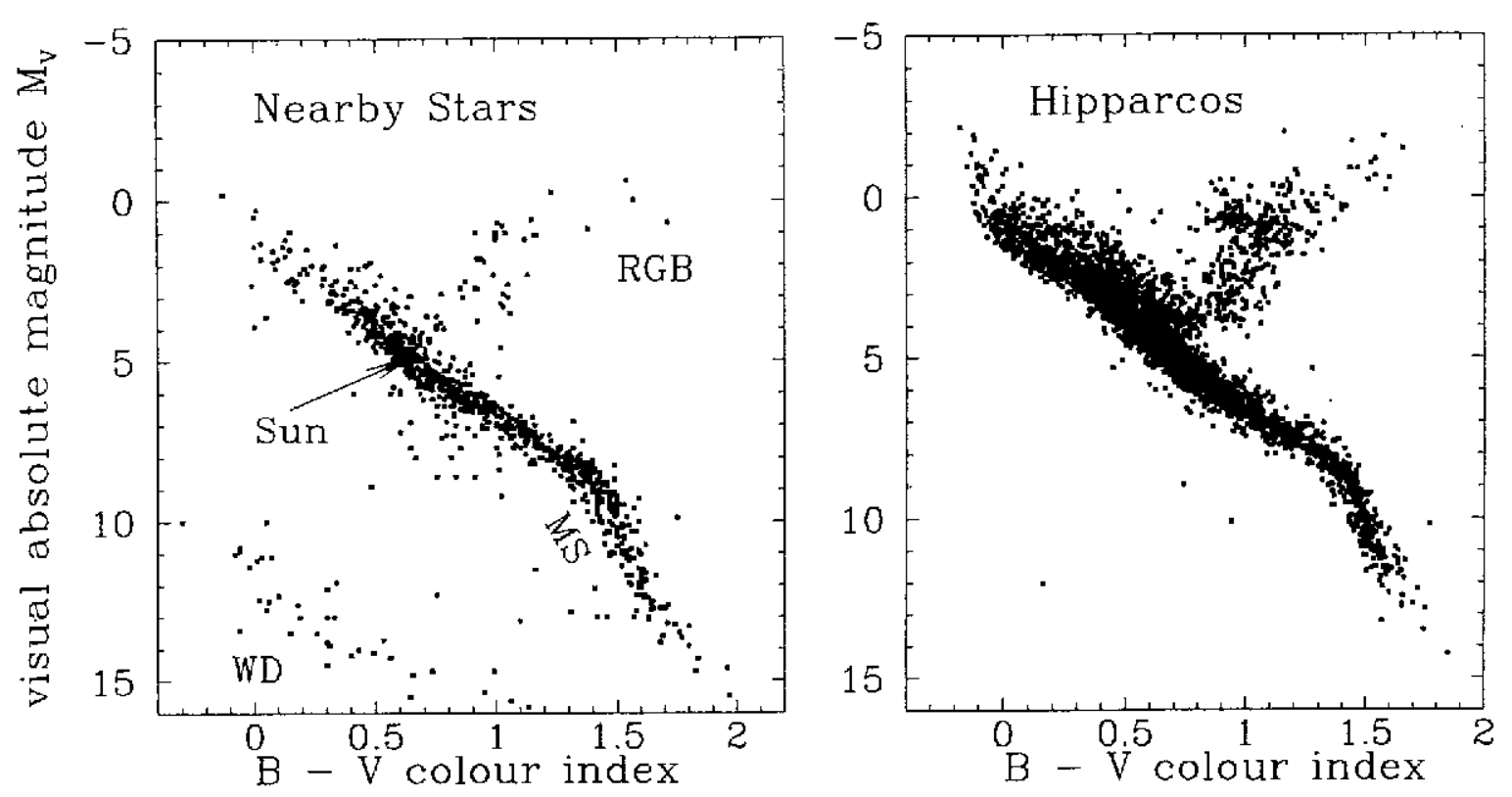

Figure 1. Deux exemples de diagrammes couleur-magnitude. Figure de gauche: pour un échantillon d'environ 1000 étoiles proches (distances inférieures à environ $20 \mathrm{pc} ; 1 \mathrm{pc}$ ou parsec $=3.086 \times 10^{13} \mathrm{~km}$ ). Figure de droite: pour un échantillon d'environ 5000 étoiles (la plupart situées à des distances comprises entre 60 et $70 \mathrm{pc}$ ) observées par le satellite HIPPARCOS. Chaque étoile de ces échantillons est représentée par un point. Les labels MS, RGB et WD se rapportent à la 'séquence principale (main sequence)', à la 'branche des géantes rouges (red giant branch)' et aux 'naines blanches (white dwarfs)': Les magnitudes représentées en ordonnée sont définies de façon à ce qu'une diminution de 5 unités corresponde à une augmentation d'un facteur 100 de la luminosité (puissance émise à la surface de l'étoile). Les étoiles les plus (moins) brillantes sont donc situées dans la partie supérieure (inférieure) des DHR. La luminosité observée (au sommet de l'atmosphère terrestre), ou 'magnitude apparente', diminue quadratiquement avec la distance stellaire. La 'magnitude absolue' est définie comme celle qui serait associée à une étoile qui serait positionnée à une distance de $10 \mathrm{pc}$. La magnitude visuelle absolue $M_{V}$ et la magnitude visuelle apparente $V$ sont associées à la brillance dans le jaune. L'abscisse porte un indice de couleur défini comme la différence entre les magnitudes apparentes dans le bleu (B) et dans le jaune (V). Plus cet indice est petit, plus l'étoile est bleue. Les étoiles les plus bleues (rouges) sont donc situées du côté gauche (droit) du DHR. Cet indice est rattaché à la 'température effective' $T_{e f f}$, définie comme la température d'un corps noir irradiant le même flux d'énergie que les étoiles considérées (voir [15] pour plus de détails)

surfaces de corrélation) est une signature directe du fait que la production d'énergie par des transmutations nucléaires est capable de contrebalancer les pertes d'énergie à la surface d'une étoile ([16]). I Ainsi, la topologie particulière du DHR met clairement la physique nucléaire au coeur de l'astrophysique stellaire; (2) la construction numérique de modèles stellaires montre que les concentrations MS, RGB, HB et AGB correspondent en fait aux phases de combustion centrale de $\mathrm{H}$, de combustion périphérique de $\mathrm{H}$, combustion centrale de $\mathrm{He}$ et combustion en couches concentriques de $\mathrm{H}$ et de $\mathrm{He}$; et (3) l'aspect différent des DHR d'amas ouverts et d'amas globulaires témoigne de la grande différence d'âge entre ces deux types d'amas, les amas globulaires étant beaucoup plus vieux que les amas ouverts.

I Les 'naines blanches (WD)' font intervenir une physique différente ne nécessitant pas une source d'énergie nucléaire 

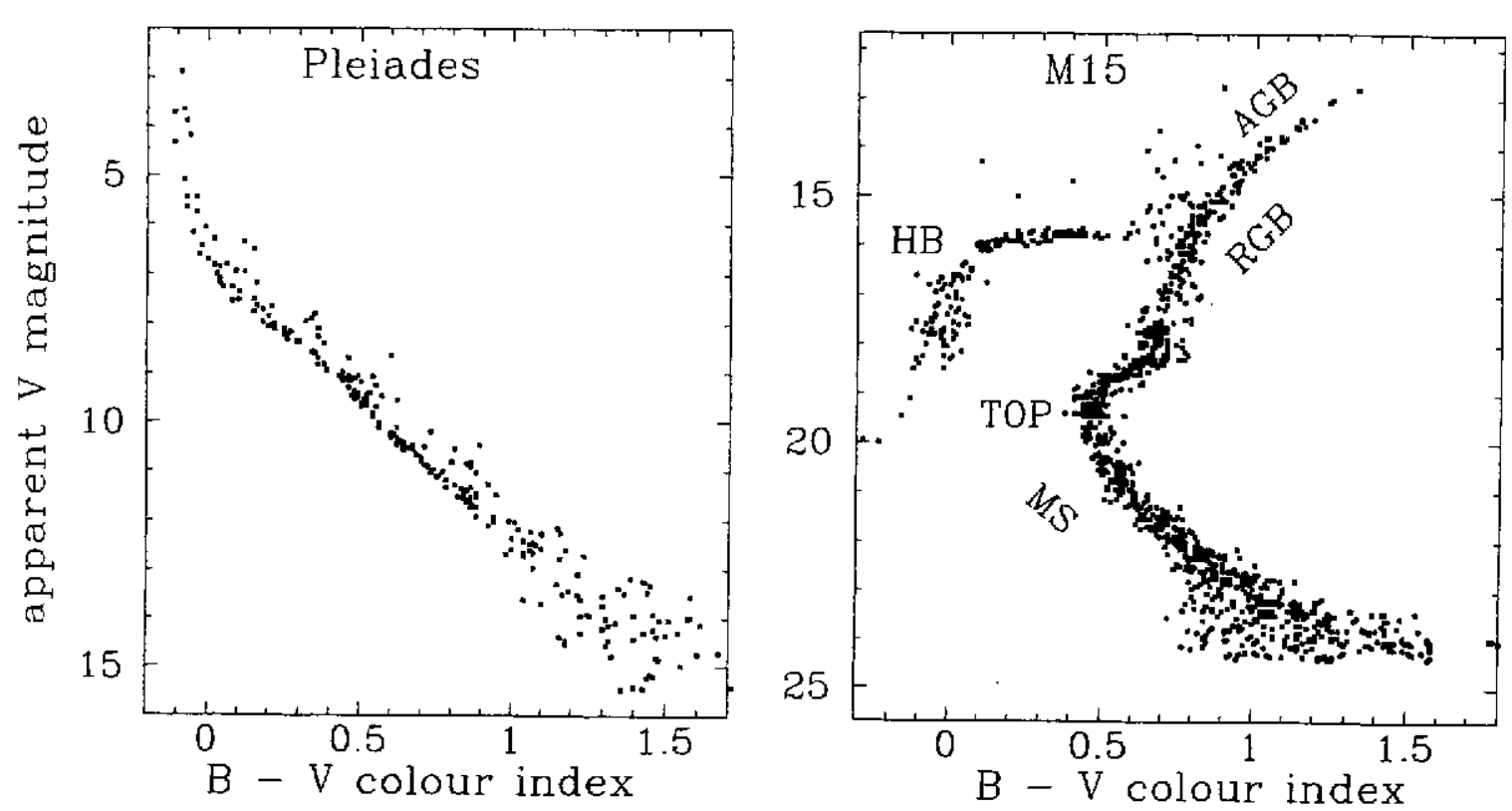

Figure 2. Même que la Fig. 1, mais pour les Pléiades, un amas galactique ('ouvert') jeune (DHR de gauche) et pour le vieil amas 'globulaire' M15 (DHR de droite). Les labels HB et AGB se rapportent à la 'branche horizontale (horizontal branch)' et la 'branche des géantes rouges asymptotiques (asympotic giant branch)'. Le label TOP identifie le sommet de la séquence principale (MS). A noter que tous les membres d'un amas ouvert ou globulaire sont en bonne approximation tous à égale distance de l'observateur, de sorte que la transformation de magnitudes apparentes en magnitudes absolues correspond simplement à une translation de l'ensemble des points des DHR d'une même quantité déterminée par le carré de la distance à l'amas

\subsection{Spectres électromagnétiques et déterminations d'abondances}

Grossièrement parlant, la lumière qui nous vient du ciel met en évidence une certaine uniformité dans la composition des objets cosmiques. Ainsi, $\mathrm{H}$ et He sont partout beaucoup plus abondants que les autres éléments dans tout l'Univers observable. Cependant, sur cette uniformité vient se greffer une grande diversité dans l'abondance des éléments et de leurs isotopes à toutes les échelles, depuis les étoiles jusqu'aux galaxies et amas de galaxies. Ceci implique l'existence de diverses classes d'objets, et même une certaine diversité parmi les objets d'une même classe. Ce qui suit ne présente brièvement que quelques-unes des très nombreuses données qui nous sont fournies aujourd'hui par l'observation astronomique.

2.2.1. L'évolution spatio-temporelle de la composition de notre Galaxie. Cette évolution peut être retracée en analysant la composition de surface d'un échantillon d'étoiles suffisamment vaste et choisi sur base de certains critères: (1) il doit contenir des étoiles d'âges différents et situées en divers endroits de la Galaxie (à diverses distances du centre galactique dans le halo ou dans le disque galactique), et (2) la surface des étoiles sélectionnées ne doit pas être contaminée par de la matière ayant subi des transformations nucléaires en leur intérieur. Cette contrainte est essentielle en vue de mettre en évidence des variations spatiales et temporelles à l'échelle galactique. Des données sur la composition du milieu interstellaire viennent très utilement compléter les données stellaires susmentionnées.

Quelques caractéristiques générales émergent du très grand nombre de données d'observation disponibles actuellement. Elles peuvent se résumer de la façon suivante: 


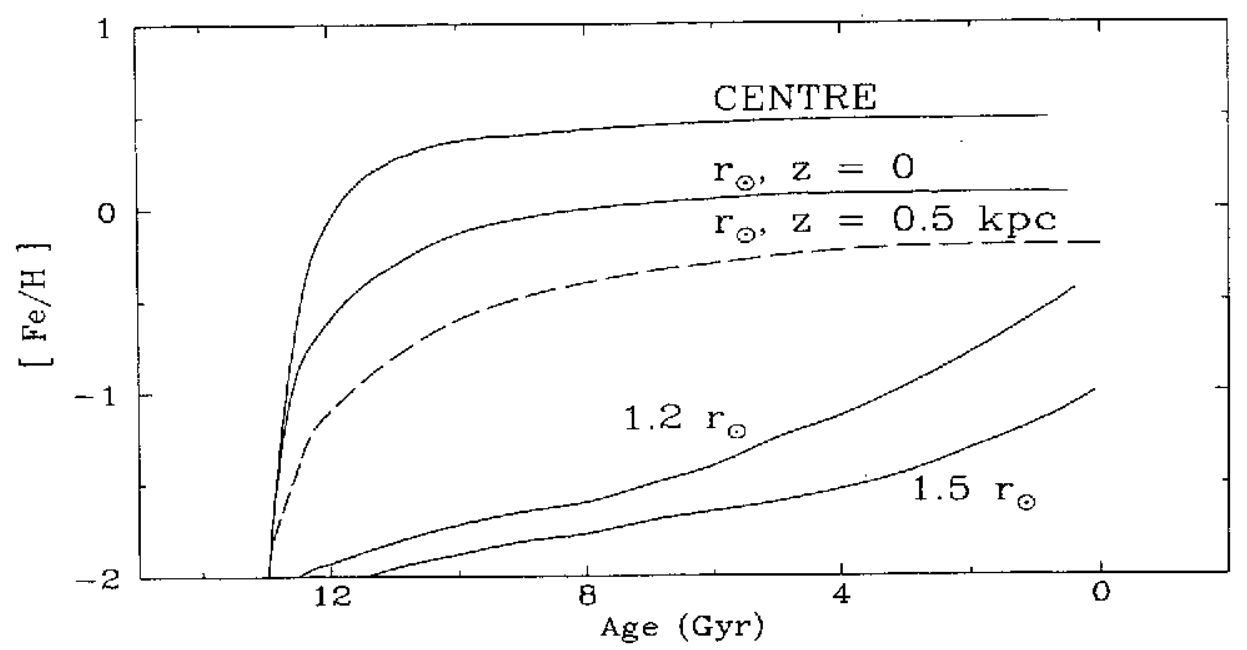

Figure 3. Représentation très schématique de la relation entre l'âge et la métallicité à 5 endroits de la Galaxie (d'après [17]): le centre galactique (CENTRE); à la distance du Soleil $r_{\odot}$ mesurée à partir du centre et dans le plan de symétrie $(z=0)$ du disque galactique; à $r_{\odot}$, mais à une distance $z=500 \mathrm{pc}$ du plan de symétrie; à $z=0$, mais à 1.2 et $1.5 r_{\odot}$. La métallicité portée en ordonnée est exprimée dans la notation spectroscopique habituelle $[\mathrm{Fe} / \mathrm{H}]=\log _{10}(\mathrm{Fe} / \mathrm{H})-\log _{10}(\mathrm{Fe} / \mathrm{H})_{\odot}$, les symboles chimiques représentant des abondances numériques et l'indice $\odot$ se référant au Soleil $\left[\log _{10}(\mathrm{Fe} / \mathrm{H})_{\odot} \approx-4.5\right]$

(1) à grande échelle, la métallicitét augmente avec le temps à une vitesse qui varie elle-même avec le temps et la position dans la Galaxie (Fig. 3);

(2) des tendances générales ainsi que des variations par rapport à l'indice de métallicité $[\mathrm{Fe} / \mathrm{H}]$ apparaissent pour un grand nombre d'éléments allant de $\mathrm{Li}, \mathrm{Be}$ et $\mathrm{B}$ d'importance cosmologique ([18]) jusqu'aux terres rares ([19]). La Fig. 4 illustre la dépendance en métallicité des nucléides appelés 'éléments $\alpha$ ' (nombre de masse $A=4 n \leq 56$ ). Ces données démontrent à nouveau que différents éléments ou groupes d'éléments s'accumulent à des vitesses différentes à différents endroits de la Galaxie. Il s'agit certainement là de la signature de différents processus nucléosynthétiques opérant plus ou moins efficacement à des moments et à des endroits différents. Il apparaît en même temps que les processus de mélange éventuels entre divers sous-systèmes galactiques (disque, bulbe, halo), ou même à l'intérieur d'un sous-système donné, n'ont pu opérer qu'avec une efficacité limitée;

(3) les mesures d'abondances dans la photosphère des étoiles sont très utilement complétées par des informations relatives à la composition du gaz et de grains de nuages interstellaires ou d'enveloppes circumstellaires. Ces observations sont effectuées dans les domaines radio ([20]) ou UV ([21]). Elles fournissent en particulier des informations très intéressantes sur l'existence ou l'absence dans le disque galactique de gradients dans la composition isotopique de certains éléments majeurs (comme $\mathrm{C}, \mathrm{N}$ et $\mathrm{O}$ ), et donc des contraintes sévères sur les modèles d'évolution chimique de la Galaxie (Sect. 3).

2.2.2. La composition d'autres galaxies Les très nombreuses mesures d'abondances dans notre Galaxie sont aujourd'hui complétées par une quantité rapidement croissante de données relatives à d'autres galaxies. Il apparaît ainsi que les galaxies, y compris celles appartenant

\footnotetext{
+ La métallicité se mesure généralement par la somme des abondances de tous les nucléides de nombre de masse $A \geq 12$. Un indicateur de métallicité souvent utilisé est l'abondance de Fe. Pour certaines classes d'étoiles, et en particlier pour les étoiles vieilles, ce choix n'est probablement pas le plus judicieux, et O peut remplacer Fe comme élément de référence
} 

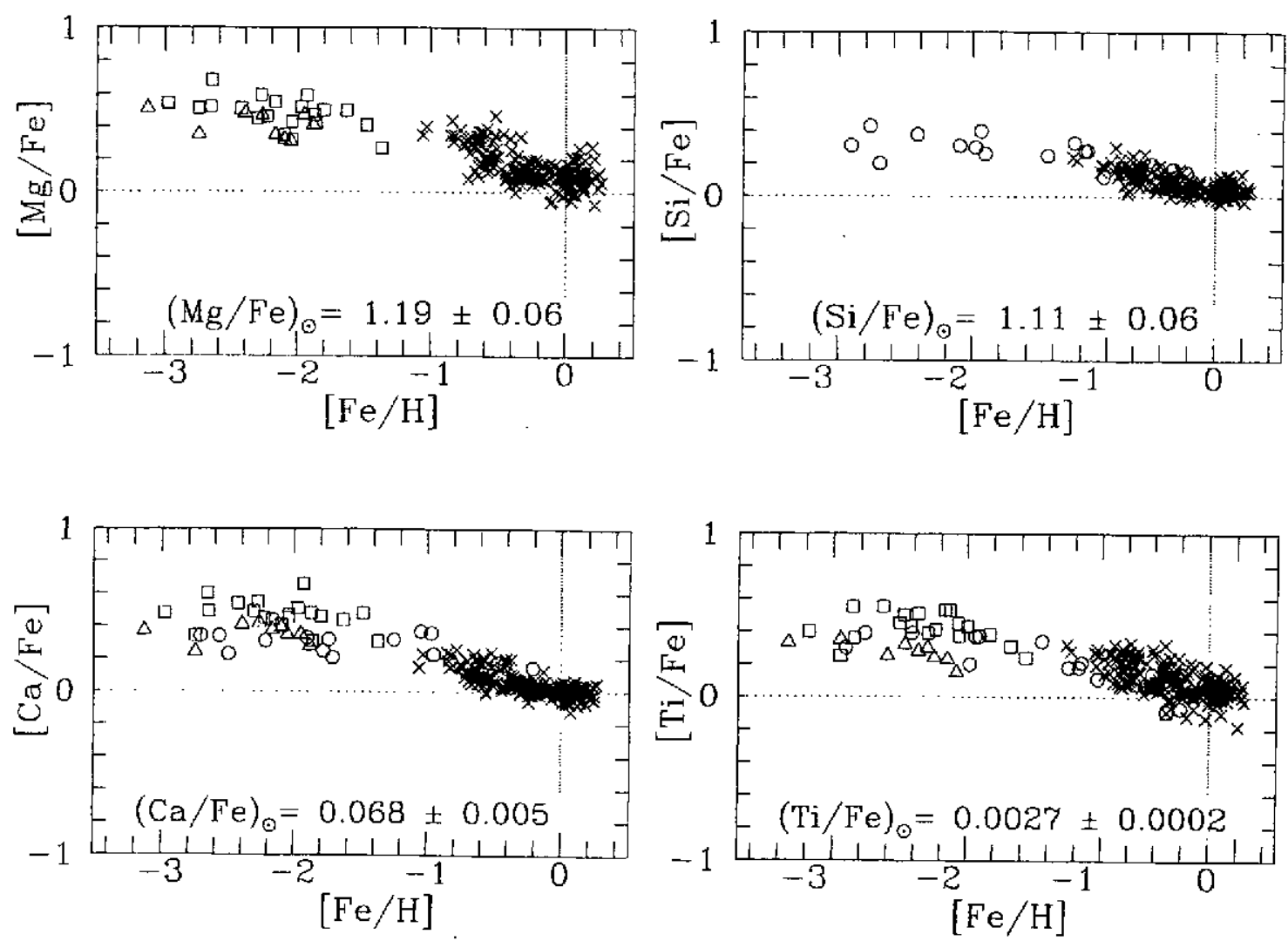

Figure 4. Variations avec la métallicité de notre Galaxie de l'abondance de divers 'éléments' $\alpha^{\prime}$. Il apparait que ces nucléides se sont accumulés plus vite que Fe. Très schématiquement, on peut dire que les étoiles à $[\mathrm{Fe} / \mathrm{H}] \lesssim-1$ peuplent le halo, alors que le disque contient celles à métallicité plus élevée

à une même classe, peuvent largement différer dans leur composition et dans la distribution spatiale de l'abondance d'un grand nombre d'éléments. Des variations très locales sont également observées dans de nombreux cas. L'une des avancées récentes les plus significatives concerne la mesure de la composition de galaxies à grand 'décalage vers le rouge' (appelées dans la littérature 'damped Lyman $\alpha$ systems' ou 'DLAs') et donc peu évoluées, les décalages vers le rouge les plus élevés correspondant en effet aux histoires évolutives les plus courtes. Une relation 'âge-métallicité' construite à partir d'un échantillon de DLAs couvrant un domaine de décalages révèle qu'un DLA d'un âge donné possède une métallicité plus faible que le disque de notre Galaxie au même âge (Fig. 5). Des données commencent à s'accumuler sur l'abondance dans les DLAs d'une variété d'éléments allant jusqu'au $\mathrm{Ni}$ ([22]). La théorie de la nucléosynthèse et les modèles d'évolution chimique des galaxies devront en rendre compte.

2.2.3. La composition d'étoiles évoluées ou en explosion Aux variations d'abondances spatio-temporelles qui existent à toutes les échelles dans les galaxies ou amas de galaxies viennent s'ajouter des différences de composition parfois importantes à l'échelle stellaire, et plus précisément dans des étoiles individuelles évoluées. Contrairement aux étoiles non évoluées auxquelles il est fait référence dans les sections précédentes, la surface d'étoiles évoluées peut être contaminée par de la matière processée nucléairement en leur intérieur à un moment ou à un autre de leur évolution. Diverses classes d'étoiles 'chimiquement particulières' sont identifiées, dont certaines se trouvent sur les branches RGB ou AGB du 

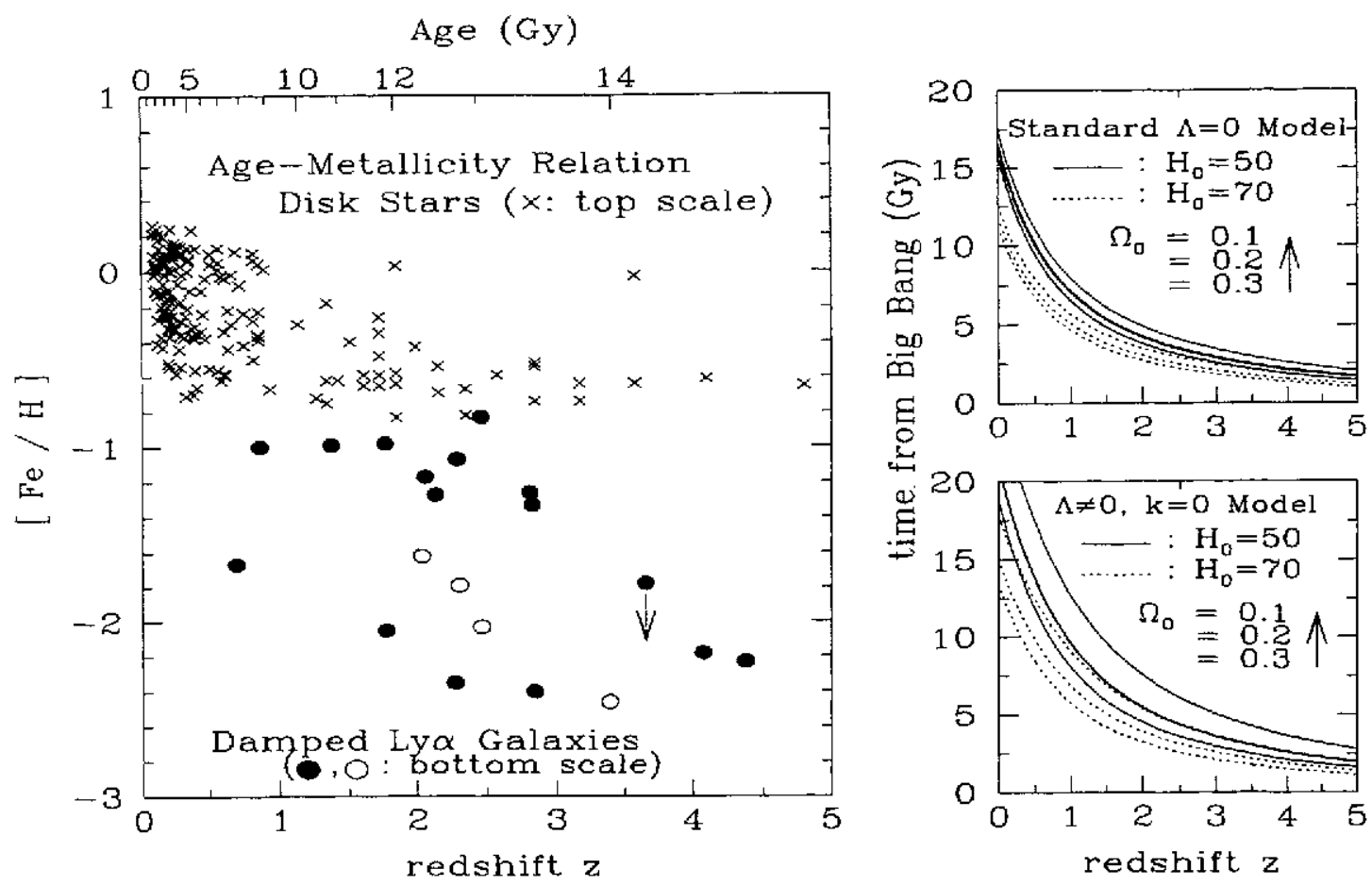

Figure 5. Figure de gauche: relations 'âge-métallicité' pour des étoiles du disque de notre Galaxie (croix se rapportant à l'axe horizontal supérieur sur lequel sont portés les âges en $10^{9}$ ans) et pour des DLAs à différents décalages vers le rouge $z$ (cercles se rapportant à l'axe horizontal inférieur). Figure supérieure de droite: relations âge-décalage $z$ dans un modèle cosmologique standard, où la constante cosmologique $\Lambda$ est supposée nulle. Figure inférieure de droite: identique à la figure supérieure, sauf que $\Lambda \neq 0$ et que l'Univers est plat $(k=0)$. Les paramètres sont la valeur actuelle $H_{0}$ de la constante de Hubble (exprimée en $\mathrm{kms}^{-1} \mathrm{Mpc}^{-1}$ ) et la densité $\Omega_{0}$ normalisée à la 'densité critique' nécessaire à la 'fermeture' de l'Univers (voir $[23,24,25]$ pour plus de détails sur les modèles cosmologiques)

DHR ([26]). Dans plusieurs cas, ces particularités de composition ne peuvent être expliquées par les modèles standards d'évolution stellaire. D'autre part, des étoiles ayant été le siège d'une explosion, comme les novae $([27])$ ou les supernovae ([28]), présentent également de multiples abondances particulières.

A ce point, il convient de noter que certaines classes d'étoiles non évoluées présentent également des particularités de composition en surface. Celles-ci ne peuvent manifestement pas être expliquées en termes de transformations nucléaires, mais sont au contraire dues à des processus de diffusion opérant dans l'atmosphère de certaines étoiles ([29]). Ainsi, l'astrophysique nucléaire doit être capable de définir ses limites avec soin, et même avec une certaine modestie!

\subsection{La composition du système solaire}

La compréhension de la composition du système solaire a toujours occupé une place privilégiée en astrophysique nucléaire. Cette situation résulte largement du fait que le système solaire fournit des abondances dont la quantité, la qualité et la cohérence sont uniques, malgré les énormes progrès réalisés dans l'observation astronomique. Ceci concerne tout particulièrement la composition isotopique de tous les éléments, qui constitue l'empreinte la plus directe de processus nucléaires. 


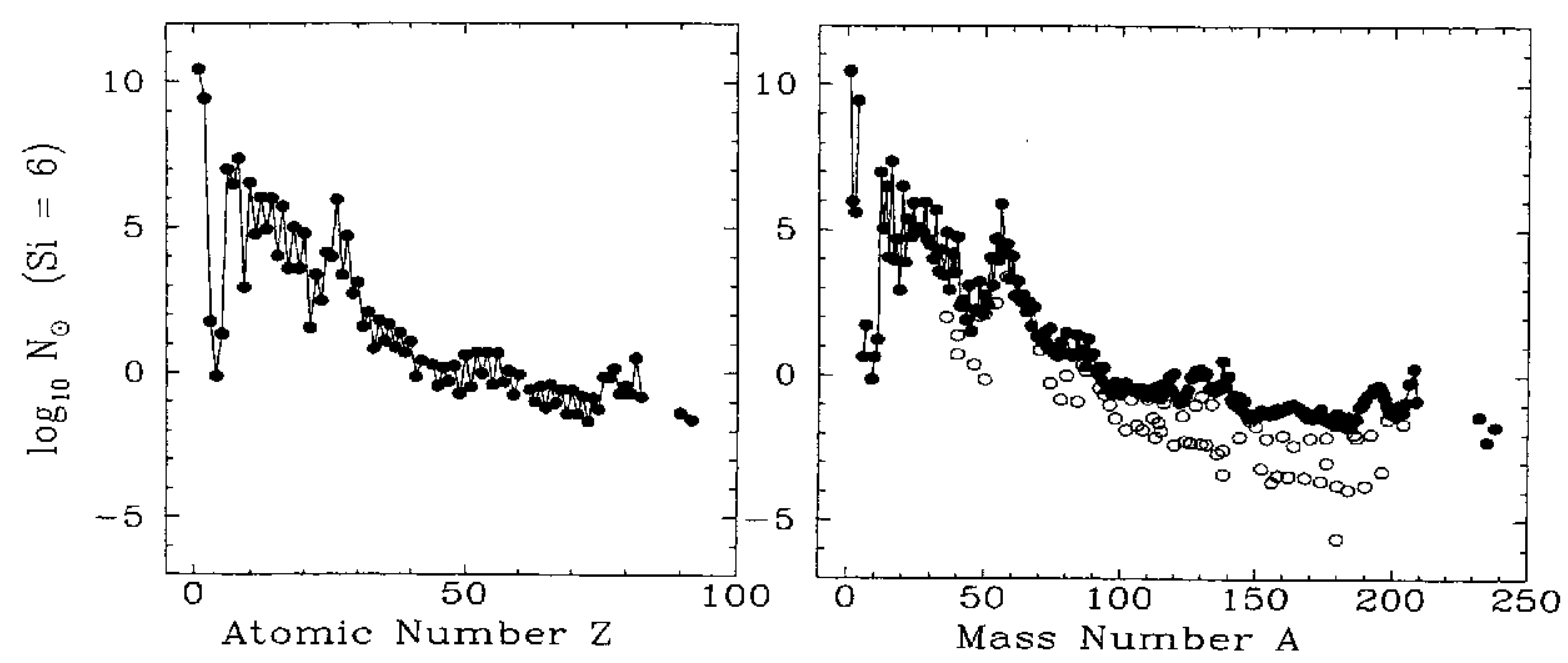

Figure 6. Abondances des éléments (figure de gauche) et des nucléides (figure de droite) considérées comme représentatives du matériau galactique à partir duquel le sytème solaire s'est formé. Les abondances sont normalisées à $10^{6}$ atomes de Si. Pour une valeur donnée du nombre de masse $A$, l'isobare le plus abondant est représenté par le symbole $\bullet$, alors que le symbole o se rapporte à un isobare moins abondant éventuel

2.3.1. La composition du gros de la matière solaire Pour l'astrophysique nucléaire, un jalon important a été la mise en évidence du fait que, en dépit de grandes différences dans l'abondance des éléments constituant les corps solides du système solaire, il était possible, après diverses corrections de nature physico-chimique, de définir un ensemble cohérent d'abondances représentatives de la composition du matériau galactique à partir duquel le système solaire s'est formé voici quelque 4.6 milliards d'années. Cette distribution d'abondances est représentée à la Fig. 6. Elle est largement basée sur la composition d'une classe spéciale de météorites très rares, les chondrites carbonnées de type CI1, qui sont considérées comme les échantillons les moins altérés de la matière solaire primitive actuellement disponibles ([30]). La spectroscopie solaire, qui est aujourd'hui en mesure de fournir des données en bon accord avec l'analyse de ces chondrites, est une source d'information privilégiée concernant l'abondance des éléments volatils $\mathrm{H}, \mathrm{He}, \mathrm{C}, \mathrm{N}, \mathrm{O}$ et $\mathrm{Ne}$ [31]. Dans quelques cas (Ar, $\mathrm{Kr}, \mathrm{Xe}, \mathrm{Hg}$ ), l'évaluation des abondances dans le système solaire ne peut être obtenue ni par les analyses météoritiques, ni par la spectroscopie solaire, et repose uniquement sur des interpolations fondées sur des considérations nucléosynthétiques.

Les processus physico-chimiques susceptibles d'expliquer les différences dans l'abondance des éléments présents dans les corps solides du système solaire ne semblent pas être capables d'affecter la composition isotopique des éléments, sauf dans quelques cas particuliers (par ex. $\mathrm{H}$ et les gaz rares). Il en résulte une grande homogénéité dans la composition isotopique du gros de la matière du système solaire. C'est pourquoi il apparaît raisonnable de prendre le matériau terrestre comme standard pour la composition isotopique des éléments dans la nébuleuse solaire primitive. La distribution de l'abondance des nucléides résultant de ce choix est aussi représentée à la Fig. 6 .

Sans entrer dans les détails, quelques caractérististiques de la Fig. 6 méritent d'être notées. En particulier, $\mathrm{H}$ et $\mathrm{He}$ sont de loin les éléments les plus abondants, tandis que $\mathrm{Li}, \mathrm{Be}$ et $\mathrm{B}$ sont largement sous-abondants par rapport aux nucléides voisins. D'autre part, quelques pics d'abondance viennent se greffer sur une courbe qui décroît avec le nombre de masse $A$ croissant. A part le pic le plus élevé centré sur ${ }^{56} \mathrm{Fe}$ (le 'pic du fer'), d'autres maxima 
locaux d'abondance se présentent aux éléments $\alpha$. De plus, un pic assez large est observé dans la région $A \approx 80-90$, tandis que des pics doubles se manifestent à $A=130-138$ et $195-208$.

Il a été rapidement noté que les distributions de la Fig. 6 démontrent clairement l'existence d'une corrélation entre les abondances dans le système solaire et les propriétés nucléaires. Par exemple, un nucléide est plus abondant que ses voisins s'il est plus stable au sens de la physique nucléaire. La compréhension de cette corrélation a été l'un des thèmes fondateurs de l'astrophysique nucléaire et divers processus ont été imaginés afin de rendre compte de la composition du système solaire. En un mot, les nucléides légers $(A<12)$ sont produits dans le Big Bang (Sect. 5) et/ou par réactions de 'spallation' (Sect. 3), tandis que les nucléides plus lourds sont essentiellement le résultat de transmutations nucléaires à l'intérieur des étoiles. Comme discuté plus en détail aux Sects. 7 et 9, différentes réactions entre particules chargées produisent l'essentiel des nucléides jusqu'au pic du fer. Au contraire, des chaînes de captures de neutrons appelées processus s et $\mathrm{r}$ interviennent pour synthétiser les espèces nucléaires plus lourdes. Un mécanisme supplémentaire, appelé processus p, est dominé par des réactions de photodésintégration de noyaux pré-existants (pour une discussion des processus s, $r$ et $p$, voir S. Goriely, ce volume).

2.3.2. Anomalies isotopiques dans la composition du système solaire La composition du système solaire a suscité un important regain d'intérêt suite à la découverte qu'une minuscule fraction de sa matière contient une variété d'éléments (du $\mathrm{C}$ au $\mathrm{Nd}$ ) dont la composition isotopique diffère de celle caractérisant le gros de la matière du système solaire. De telles 'anomalies isotopiques' ont été découvertes dans des inclusions de météorites primitives ([32]), ainsi que dans divers types de grains météoritiques (diamant, graphite, $\mathrm{SiC}$, etc) ([33]). Les inclusions se sont solidifiées à partir d'un matériau hors d'équilibre avec le reste de la nébuleuse solaire, alors que les grains ont sans doute une origine circumstellaire et ont survécu à toutes les péripéties ayant accompagné leur inclusion dans les météorites.

Ces anomalies éclairent d'un jour nouveau de nombreuses questions d'astrophysique, comme la physique et la chimie des grains interstellaires, la formation et la croissance de grains autour d'étoiles nucléosynthétiquement actives, les circonstances dans lesquelles les étoiles (et en particulier des structures semblables au système solaire) peuvent se former, ainsi que l'histoire de la prime jeunesse du Soleil et des corps solides du système solaire. Enfin, elles soulèvent la question de leur(s) origine(s) nucléosynthétique(s) et offrent la très intéressante perspective de disposer d'informations relatives à un très petit nombre d'événements nucléosynthétiques (peut-être même un seul). Cette situation diffère totalement de celle rencontrée dans l'étude du gros de la matière solaire. Celle-ci résulte en effet du mélange des produits provenant d'un grand nombre d'agents nucléosynthétiques et requiert par conséquent la modélisation de l'évolution chimique de la Galaxie (Sect. 3).

La découverte que certaines des anomalies observées sont dues à la désintégration dans le système solaire lui-même de radionucléides de demi-vie excédant $10^{5}$ ans environ, et tout particulièrement de ${ }^{26} \mathrm{Al}$ ([34]), n'a fait qu'accroître encore l'intérêt des astrophysiciens pour les anomalies isotopiques. La présence de radionucléides de période relaivement courte dans le système solaire a en effet d'importantes implications au niveau de notre compréhension de sa (pré)histoire et de l'identification des sources stellaires possibles de ces radionucléides.

2.3.3. La composition du rayonnement cosmique et des particules énergétiques du Soleil Beaucoup de progrès ont été réalisés ces dernières années dans notre connaissance de l'abondance des éléments et des isotopes des particules énergétiques du Soleil ([35]), ainsi 


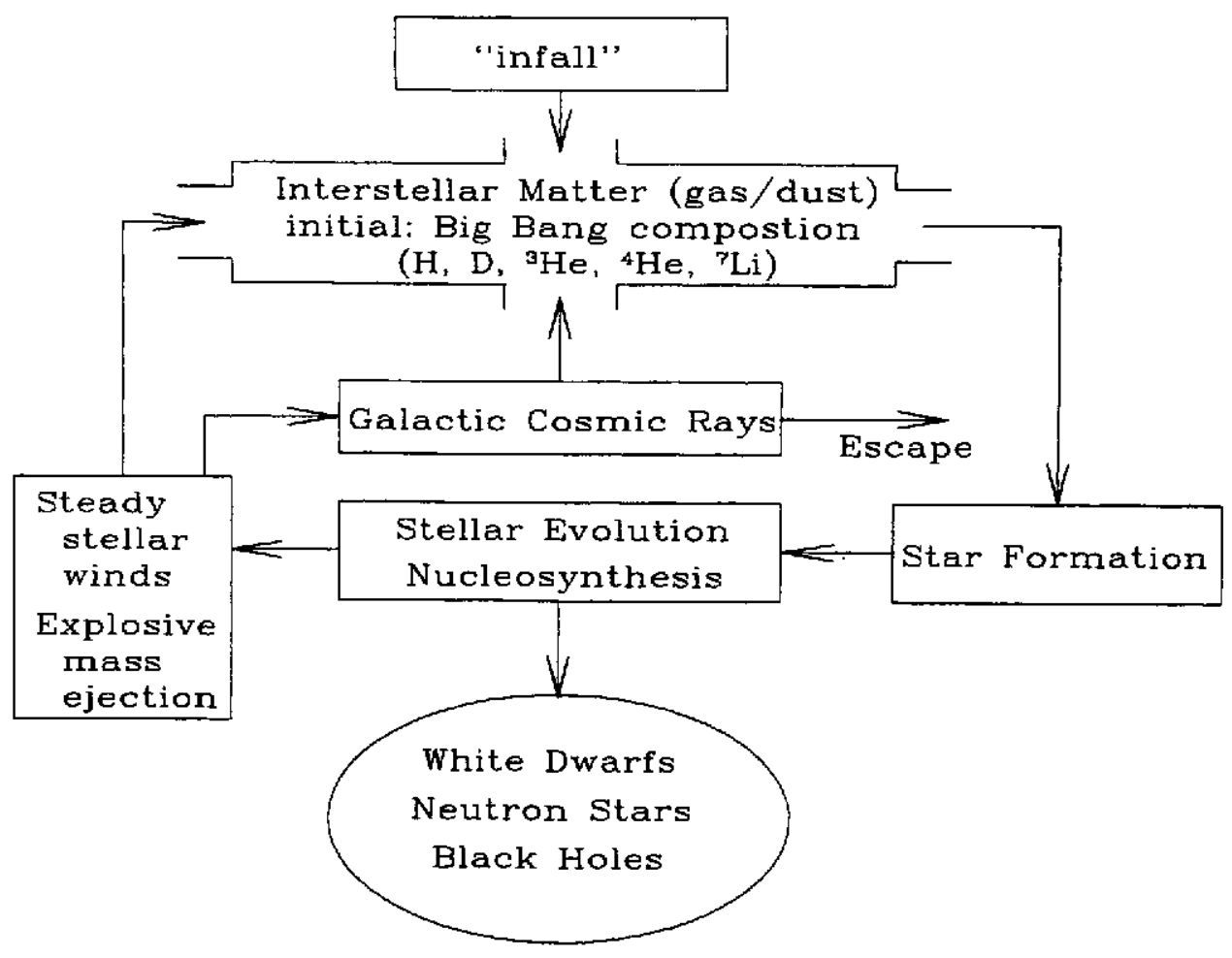

Figure 7. Schéma très simplifié du grand 'mélangeur' galactique (voir texte)

que du rayonnement cosmique galactique $([36,37])$. Ces données ont permis de mieux cerner la nature des sources possibles du rayonnement cosmique et de leur composition à la source. En même temps, elles ont suscité quelques études en astrophysique nucléaire.

\section{Les pourvoyeurs de nucléides dans l'Univers: synopsis}

La modélisation de l'évolution de la composition des galaxies (généralement appelée l'évolution 'chimique' des galaxies) est clairement l'un des problèmes les plus complexes auquel doit faire face l'astrophysique, en raison notamment du nombre extrêmement élevé d'événements nucléosynthétiques très variés concourant à chaque instant et en chaque lieu à des variations plus ou moins substantielles de l'abondance d'une diversité plus ou moins grande de nucléides.

La question de l'évolution chimique des galaxies a été abordée à divers niveaux de sophistication, depuis des modèles dits 'dynamico-chimiques' jusqu'à de simples modèles 'à une zone'. Les premiers tentent de coupler la dynamique et la thermodynamique des galaxies à leur 'chimie', dont la description est cependant très rudimentaire en raison de la complexité des autres effets pris en compte. Au contraire, les modèles du second type négligent totalement les aspects dynamiques et thermodynamiques pour se limiter à une description plus ou moins simplifiée des variations de composition dans le voisinage solaire. Il n'est pas possible dans le cadre de ce cours de discuter de façon détaillée du problème de l'évolution chimique des galaxies, et nous en présentons seulement une image extrêmement simplifiée (voir $[12,38]$ pour plus de détails).

La façon dont une galaxie évolue chimiquement est représentée schématiquement à la Fig. 7. Considérons le milieu interstellaire (MIS) (fait de gaz et de poussières) juste après le 
processus de formation d'une galaxie. La composition du MIS à ce moment est supposée être celle résultant du Big Bang standard, qui prédit la production en quantités significatives de $\mathrm{H}, \mathrm{D},{ }^{3} \mathrm{He},{ }^{4} \mathrm{He}$ et ${ }^{7} \mathrm{Li}$ seulement (Sect. 5). Une partie du matériau de ce MIS est incorporé dans les étoiles qui se forment et qui, au fil de leur évolution et des nombreuses réactions nucléaires associées, vont plus ou moins profondément transformer la composition initiale d'une fraction au moins de leur matériau initial. Au cours de cette évolution, l'étoile est susceptible, grâce à divers mécanismes (Sect. 6), de retourner au MIS une partie plus ou moins importante de leur matière chimiquement modifiée, le reste étant piégé dans des 'résidus' stellaires (naines blanches, étoiles à neutrons ou même trous noirs). Ces résidus ne participent normalement plus à l'évolution chimique des galaxies, du moins quand ils ne sont pas membre d'un système binaire. Il faut aussi se rappeler qu'une infime fraction de la matière éjectée par certaines étoiles peut être accélérée et atteindre des énergies typiques du rayonnement cosmique galactique. Ces particules énergétiques peuvent interagir avec le MIS et induire ainsi des réactions de spallation susceptibles de produire une fraction significative $\mathrm{du} \mathrm{Li}, \mathrm{Be}$ et $\mathrm{B}$ des galaxies, et en particulier du disque de notre Voie Lactée. Du moins dans les galaxies spirales comme la nôtre, une fraction du rayonnement cosmique pourrait s'échapper du disque, de même d'ailleurs qu'une fraction de la matière stellaire éjectée lors d'explosion, en particulier de type supernova (cette possibilité n'est pas représentée à la Fig. 7). Au contraire, de la matière du halo galactique, de composition éventuellement proche de celle provenant du Big Bang, pourrait continuer à s'accumuler sur le disque ('infall') bien après l'épisode principal de formation galactique.

Un ingrédient de base des modèles d'évolution chimique des galaxies est la 'fonction de création stellaire', c-à-d le nombre d'étoiles formées par unité de surface du disque galactique (dans des galaxies spirales) par intervalle unité de masses stellaires et de temps. Cette question a été discutée en grands détails par [39]. Sur base de considérations phénoménologiques et pour d'évidentes raisons de facilité, la fonction de création stellaire est généralement supposée séparable en une fonction du temps seulement (la 'vitesse de formation stellaire') et en une fonction de la masse des étoiles seulement (la 'fonction de masse initiale' ou FMI). Une telle FMI basée sur des données d'observations dans le voisinage solaire est représentée à la Fig. 8. L'une des caractéristiques essentielles de cette fonction (du moins dans le voisinage solaire, mais apparemment aussi dans des situations plus générales) est sa décroissance rapide avec l'augmentation des masses stellaires, du moins dans le domaine $M \gtrsim M_{\odot}$.

Un autre ingrédient majeur des modèles d'évolution chimique des galaxies est la masse et la composition de la matière éjectée par une étoile de masse initiale donnée. Cette quantité est appelée le 'rendement' (de l'anglais 'yield'). Son évaluation nécessite la modélisation de l'évolution d'étoiles dans un large domaine de masses initiales (essentiellement entre 1 et 100 $M_{\odot}$ environ), ainsi que de la nucléosynthèse associée. Les mécanismes d'éjection de matière dans le MIS doivent également être connus pour chaque étoile. Ces processus, ainsi que la fraction de la masse stellaire restituée au MIS sont résumés à la Fig. 9.

Notons enfin que quelques modèles d'évolution chimique tentent de prendre en compte de façon très approchée la contribution spécifique de certaines étoiles binaires. Ceci concerne en particulier l'enrichissement galactique dû à des novae ou à certains types de supernovae (Type Ia) trouvant leur origine dans l'accrétion par des naines blanches de matière en provenance de leur compagnon. 


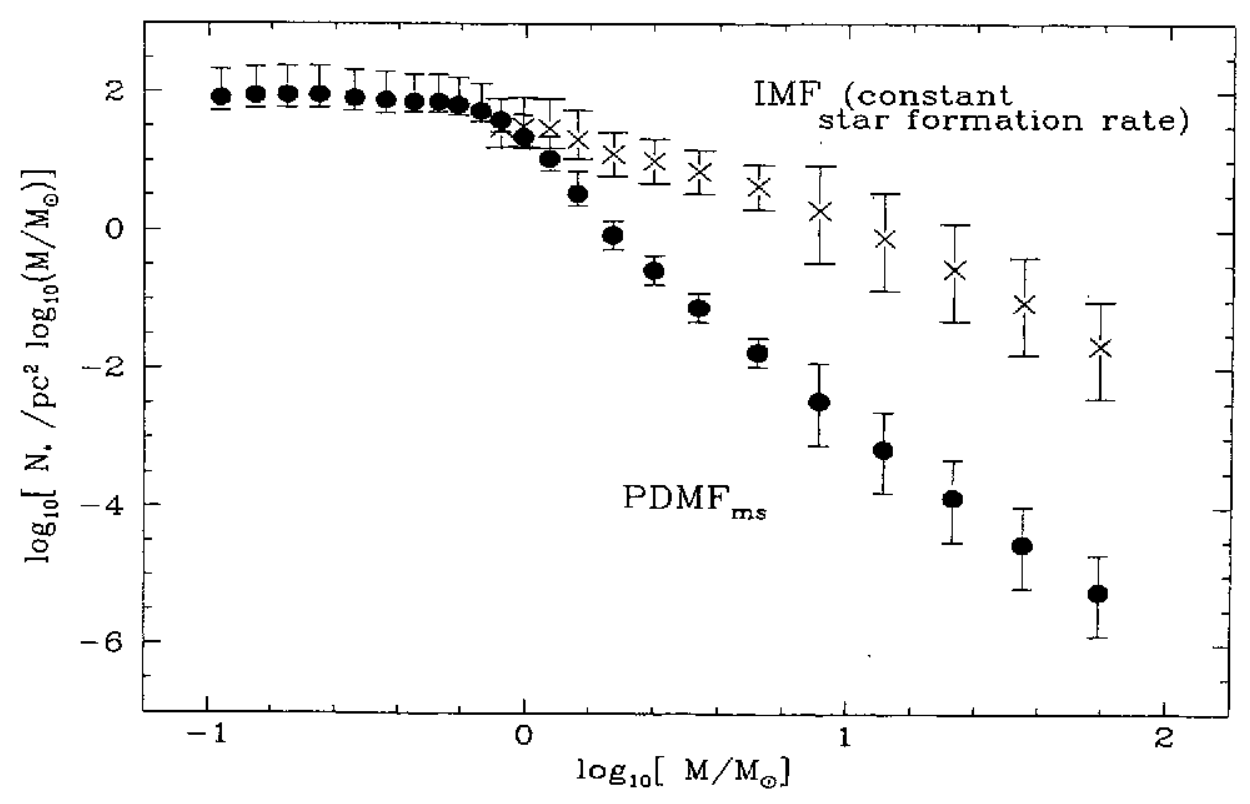

Figure 8. Une fonction de masse initiale FMI pour le voisinage solaire du disque galactique. Elle est obtenue à partir de la 'fonction de masse actuelle' ('present-day mass function ou PDMF') pour des étoiles de la séquence principale (MS) sous l'hypothèse que la fonction de création stellaire ne varie pas dans le temps. La PDMF $_{m s}$ fournit le nombre d'étoiles qui sont sur le point de quitter la MS dans le DHR. La différence entre la PDMF ms $_{\text {et la FMI }}$ reflète le rapport de la durée de vie des étoiles de la MS et de l'âge de la Galaxie (supposé être entre 9 et 15 milliards d'années)

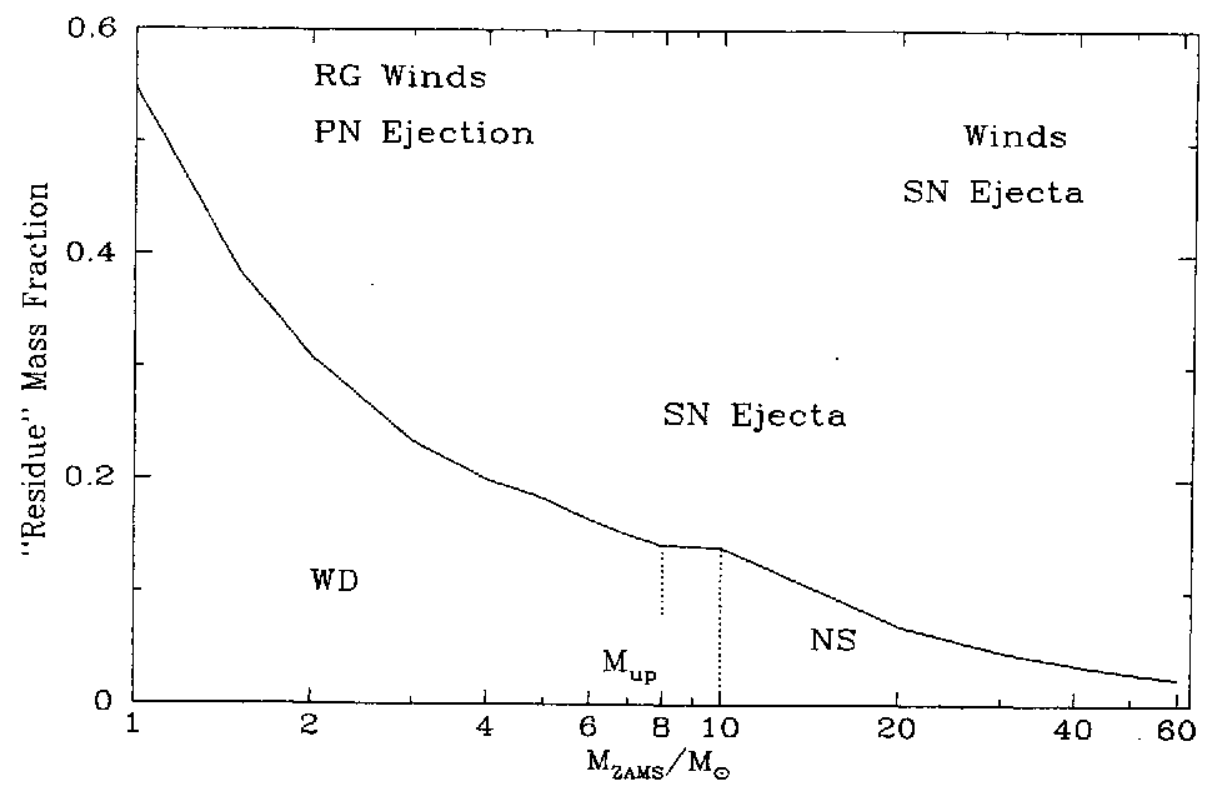

Figure 9. Représentation schématique de la fraction de la masse initiale $M_{\text {ZAMS }}$ (ZAMS pour 'zero-age main sequence') d'une étoile isolée dont l'évolution se termine par la production d'un résidu de type naine blanche (WD) ou étoile à neutrons (NS). Le reste de l'étoile est éjecté dans le MIS par le mécanisme indiqué (RG, PN et SN se rapportent aux étoiles géantes rouges, nébuleuses planétaires et supernovae). Les étoiles de masse initiale inférieure à $M_{\mathrm{up}} \approx 8 M_{\odot}$ sont supposées conduire à des naines blanches à la fin de leur évolution, alors que celles pour lesquelles $M \gtrsim 10 M_{\odot}$ laissent une étoile à neutrons de $1.4 M_{\odot}$. L'évolution des étoiles dans le domaine $8 \lesssim M \lesssim 10 M_{\odot}$ est encore mal connue. Elles pourraient terminer leur évolution en laissant une naine blanche, une étoile à neutrons, ou peut-être même ne pas laisser de résidu. Le shéma présenté est encore sujet à de très nombreuses incertitudes 


\section{La physique nucléaire dans les pourvoyeurs de nucléides: synopsis}

Ainsi que suggéré dans les sections précédentes, la physique nucléaire marque de son empreinte l'Univers à toutes les échelles. Afin de déchiffrer les messages du cosmos qui peuvent intéresser l'astrophysique nucléaire, il est indispensable de partir d'une description aussi fiable et complète que possible des propriétés fondamentales des noyaux atomiques dans les conditions du laboratoire, et tout particulièrement de leurs masses et leurs modes de désintégration. Si cette information est nécessaire au développement de l'astrophysique nucléaire, elle n'est en aucune façon suffisante. Il reste en effet à découvrir comment les propriétés nucléaires mises à jour sur terre peuvent éventuellement être affectées dans les situations astrophysiques où peuvent régner des conditions très variées, et en particulier de très hautes températures et/ou densités ne pouvant absolument pas être atteintes en laboratoire. Ces questions ont été au centre de très nombreuses recherches, tant expérimentales que théoriques.

\subsection{La masses des noyaux 'froids'}

Les masses nucléaires (ou, de façon équivalente, les énergies de liaison ou de séparation) jouent un rôle essentiel dans tous les chapitres de l'astrophysique nucléaire. Leur connaissance est en effet indispensable à l'évaluation des probabilités de toute transformation nucléaire et donc au calcul de l'énergétique associée.

La Fig. 10 présente les quelque 2500 nucléides qui ont été identifiés à ce jour dans le laboratoire. Parmi eux, 286 environ existent dans la nature, alors que les autres sont uniquement produits en laboratoire. Malgré son étendue, cet ensemble de noyaux est loin de rencontrer les besoins de l'astrophysique. C'est particulièrement le cas dans le traitement du processus $\mathrm{r}$ (voir S. Goriely, ce volume), qui nécessite la prise en compte d'un très grand nombre de noyaux encore impossible à produire expérimentalement. La théorie doit donc impérativement compléter les efforts expérimentaux.

\subsection{Les noyaux 'chauds'}

Les conditions particulières régnant dans les sites astrophysiques, et spécialement dans les intérieurs stellaires, apportent leur lot de difficultés supplémentaires et souvent d'une grande complexité. Même les notions fondamentales de 'liaison' nucléaire ou de stabilité doivent être traitées avec grand soin dans certaines situations. Ceci vient en particulier du fait que les noyaux plongés dans un milieu à très haute température se trouvent non seulement dans leur état fondamental, mais également dans un ensemble de niveaux excités. Ceuxci sont peuplés par interaction avec des particules (principalement des électrons provenant de l'ionisation des atomes) ou des photons. Dans les intérieurs stellaires (même dans des conditions explosives), l'équilibre thermodynamique est en général réalisé à un très haut niveau de précision, du moins localement, de sorte que les populations relatives des niveaux nucléaires sont fournies avec une excellente approximation par la distribution statistique de Maxwell-Boltzmann ([16]). D'après cette loi, la population thermique de niveaux excités devient significative si la température $\left[k T \approx 8.6 \times\left(T / 10^{8} \mathrm{~K}\right) \mathrm{keV}\right]$ devient commensurable avec l'énergie d'excitation. A une température donnée, les niveaux à basse énergie d'un noyau lourd impair-impair sont donc particulièrement peuplés.

Les états isomériques posent un certain nombre de problèmes spécifiques, leur population pouvant différer plus ou moins fortement des valeurs d'équilibre à certaines températures. Ces populations d'équilibre peuvent en effet ne pas avoir le temps d'être atteintes en 


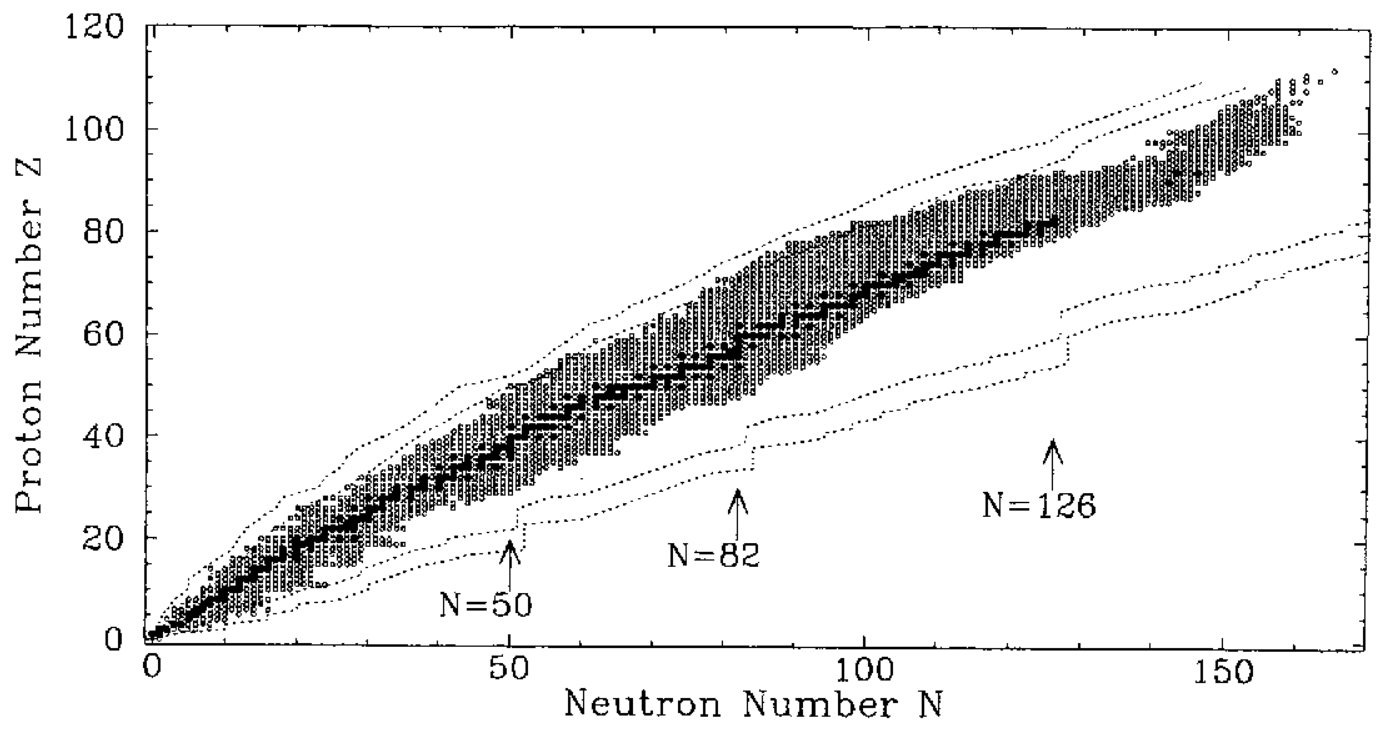

Figure 10. 'Carte de Segrè' des nucléides stables ou à longue période $\left(t_{1 / 2} \gtrsim 4.6 \times 10^{9}\right.$ ans, qui est l'âge approximatif du système solaire) (points noirs). Le radionucléide ${ }^{234} \mathrm{U}$ $\left(t_{1 / 2} \approx 2.5 \times 10^{5}\right.$ ans) n'est pas représenté, même s'il se trouve également à l'état naturel sur terre suite à son appartenance à la chaîne de désintégration de ${ }^{238} \mathrm{U}$. Les cercles ouverts représentent des noyaux produits en laboratoire dont la demi-vie, et dans bien des cas la masse, sont connues. Les nucléides stables forment la 'vallée de stabilité nucléaire' (parfois appelée 'vallée de Heisenberg'). Les noyaux situés à droite (gauche) de cette ligne sont qualifiés de riches (déficients) en neutrons, et se transforment spontanément par désintégration $\beta$. Les nucléides très lourds peuvent en plus subir des désintégrations $\alpha$ ou fissionner. Assez loin de la vallée de stabilité, les noyaux peuvent même devenir instables vis-à-vis de l'émission d'un neutron ou d'un proton (observée en laboratoire dans quelques cas). L'ensemble des noyaux dont les énergies de séparation neutronique ou protonique tendent vers zero définissent les 'lignes de drip' en neutrons et protons (lignes en pointillés) prédites par un modèle de masses nucléaires. Des lignes de drip doubles sont représentées pour rendre compte des effets pair-impair (A noter qu'une énergie de séparation protonique négative n'implique pas nécessairement une émission spontanée de protons en raison de la présence d'une barrière coulombienne)

raison de la probabilité relativement faible des transitions- électromagnétiques entre états isomérique et fondamental qui sont plus ou moins fortement 'interdites' en raison des règles de sélection auxquelles obéissent ces transitions. Des situations de ce type présentant un intérêt astrophysique concernent, par exemple, ${ }^{26} \mathrm{Al}([40]),{ }^{176} \mathrm{Lu}([41])$ et ${ }^{180} \mathrm{Ta}([42])$. Dans ces divers cas, la thermalisation ne peut en général s'opérer que si l'état isomérique peut être peuplé à partir du fondamental par l'intermédiaire d'un état à plus haute énergie dont le spin est intermédiaire entre celui du fondamental et de l'isomérique. En raison de son énergie d'excitation, cet état intermédiaire ne peut être significativement peuplé qu'à température suffisamment élevée. Ainsi, l'état isomérique à $228 \mathrm{keV}$ de ${ }^{26} \mathrm{Al}\left(t_{1 / 2}=6.3 \mathrm{~s}\right)$ est prédit ne pouvoir atteindre un équilibre thermique avec l'état fondamental qu'à des températures excédant environ $5 \times 10^{8} \mathrm{~K}$.

La présence dans un plasma stellaire de noyaux dans leur état fondamental aussi bien que dans leurs états excités a d'importantes conséquences sur la probabilité des divers modes de désintégration ou de transmutation nucléaire, et par conséquent sur différents processus nucléosynthétiques. Dans de nombreux cas, la connaissance de la masse d'un noyau dans son état fondamental n'est donc pas suffisante et doit être complétée par l'évaluation de 'fonctions de partition nucléaire', c-à-d des sommes des populations d'équilibre des divers 
niveaux excités (en excluant éventuellement les états isomériques). De telles fonctions interviennent notamment lorsqu'une réaction nucléaire est en équilibre avec sa réaction inverse, une situation extrême de ce type étant un état 'd'équilibre nucléaire statistique (ENS)' (Sect. 7.3).

Le fait que des noyaux excités jouent un rôle dans de nombreuses questions d'astrophysique nucléaire rend indispensable la connaissance de leur spectre d'énergie et de la séquence des spins des niveaux impliqués. Une telle information est rarement disponible expérimentalement, tout particulièrement pour des noyaux exotiques, ou même dans le cas de noyaux stables lorsque des températures très élevées doivent être considérées. Dans de telles conditions, des niveaux d'énergie relativement élevée peuvent en effet être assez abondamment peuplés.

\subsection{Noyaux à haute densité}

Aux densités élevées rencontrées notamment dans les supernovae ou les étoiles à neutrons, la notion de liaison nucléaire ne peut prendre son sens qu'en terme d'une équation d'état nucléaire (EEN) qui décrit la densité d'énergie et la pression d'un système de nucléons et/ou de noyaux en fonction de la densité de la matière.

A des densités $\rho \lesssim 10^{-2} \rho_{0}$, où $\rho_{0} \approx 3 \times 10^{14} \mathrm{gcm}^{-3}$ est la densité de saturation nucléaire, l'ENS est en général réalisé dans les conditions astrophysiques, de sorte que l'EEN peut être obtenue à partir des lois de la mécanique statistique d'équilibre. Le problème le plus sérieux dans ce cas résulte du fait que la matière en question est en général faite de noyaux extrêmement riches en neutrons dont les fonctions de partition, sinon les énergies de liaison (qui sont des quantités essentielles entrant dans les équations de la mécanique statistique) ne sont pas connues expérimentalement.

L'EEN à des densités $\rho$ allant de 0.01 à 10 fois environ $\rho_{0}$ est l'un des ingrédients essentiels des modèles de supernova ou d'étoiles à neutrons. En particulier, les caractéristiques de l'EEN autour de $\rho_{0}$ déterminent dans une large mesure si une étoile massive peut ou non exploser en supernova. Une étude détaillée de la physique entrant dans la construction de l'EEN dans ce domaine de densités sort du cadre de ces leçons. Le lecteur intéressé peut consulter par exemple $[43,44]$.

\subsection{Les transformations nucléaires dues aux interactions faibles}

Une grande diversité de processus dus à l'interaction faible jouent un rôle de première importance dans beaucoup de questions d'astrophysique ([45]). Dans la suite, nous ne discutons brièvement que de quelques problèmes ayant des implications en évolution stellaire et en nucléosynthèse. Nous omettrons par contre des questions très intéressantes, comme la physique du neutrino et ses implications dans divers domaines de l'astrophysique et de la cosmologie.

Un exemple très spécifique de l'importance des interactions faibles est fourni par la réaction $\mathrm{H}+\mathrm{H} \rightarrow^{2} \mathrm{H}+\mathrm{e}^{+}+\nu_{e}$ qui permet l'enclenchement des chaînes proton-proton constituant le mode dominant de combustion de l'hydrogène dans des étoiles dont la masse $M \lesssim 1 M_{\odot}$ (Sect. 7.1). A des phases plus avancées de leur évolution, divers processus d'interaction faible peuvent se produire dans les étoiles, certains étant inconnus dans le laboratoire, tout comme la réaction $\mathrm{H}+\mathrm{H}$.

4.4.1. Divers modes de désintégration $\beta$ en astrophysique Les formes les plus familières de processus d'interaction faible dans le laboratoire sont la désintégration $\beta^{ \pm}$et la capture 


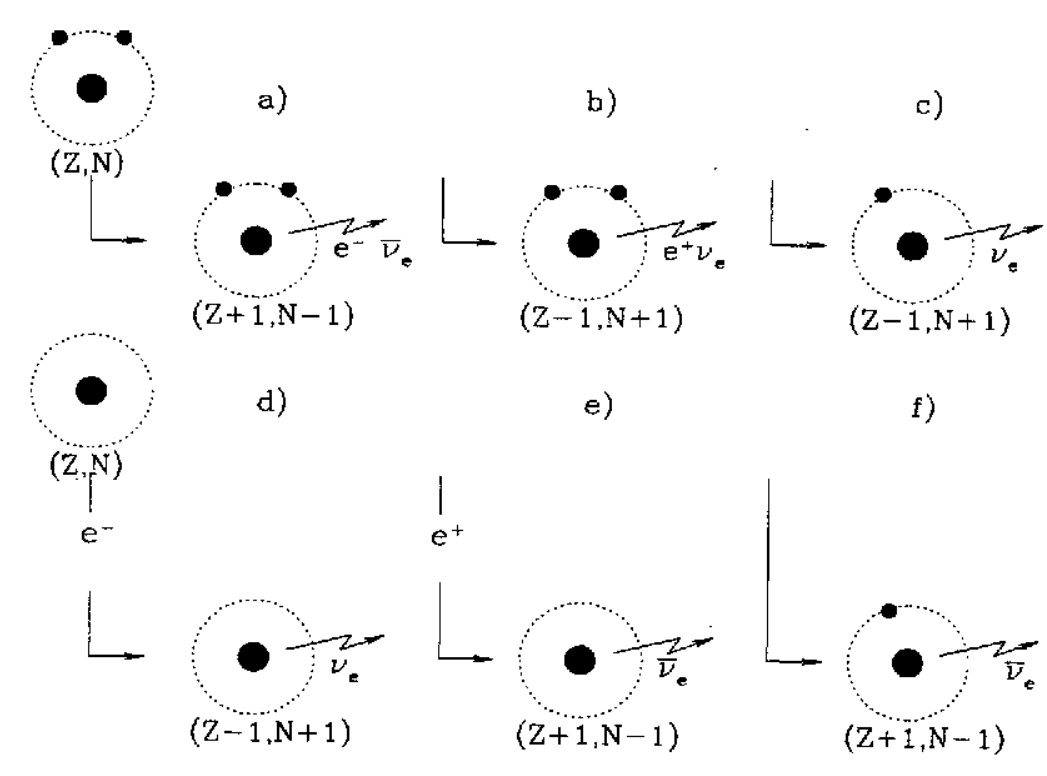

Figure 11. Schéma de différents modes de désintégration $\beta$ : (a) désintégration $\beta^{-}$, (b) désintégration $\beta^{+}$, (c) capture d'un électron orbital, (d) capture d'un électron libre, (e) capture d'un position libre et (f) création e. Les processus (a)-(c) sont les modes usuels de désintégration dans les conditions du laboratoire, tandis que les transformations (d)-(f) peuvent se produire dans les conditions stellaires

d'un électron orbital [voir Figs. 11(a)-(c)]. La probabilité de ces trois processus peut être plus ou moins différente dans le laboratoire et dans les conditions astrophysiques. L'un des effets responsables de ces différences résulte de la réduction possible de l'espace des phases accessible à l'électron émis dans une désintégration $\beta^{-}$. Cette réduction est elle-même due à la dégénérescence du gaz de Fermi-Dirac des électrons rencontré dans une variété de situations astrophysiques.* D'autres effets se rapportent à la contribution d'états excités du noyau au processus de désintégration, ainsi qu'à l'ionisation. Cette perte d'électrons libres se produit dans beaucoup de situations stellaires, principalement à haute température. Des atomes accélérés à des énergies relativistes, comme dans le cas du rayonnement cosmique galactique, se débarassent également de leurs électrons. L'ionisation peut influencer de diverses façons les demi-vies nucléaires. L'effet le plus évident est une réduction de la probabilité de capture d'électrons liés. Une conséquence moins triviale est le développement éventuel d'un processus de 'création e (bound-state $\beta$-decay)' qui consiste en la création d'un électron dans une orbite atomique ayant perdu tous ou une partie de ses électrons par ionisation [Fig. 11(f)].

D'autres modes de désintégrations $\beta$ peuvent exister dans les plasmas stellaires. La capture d'électrons libres [Fig. 11(d)] est assez courante et est souvent plus importante que la capture d'électrons liés, en particulier évidemment dans des sites où l'ionisation est importante. Les effets les plus spectaculaires de la capture d'électrons libres se manifestent lorsque le gaz d'électrons est fortement dégénéré. Dans ces conditions en effet, des noyaux très stables dans le laboratoire peuvent devenir instables si l'énergie de Fermi des électrons

* Dans des conditions d'équilibre thermodynamique local qui prévalent dans la plupart des intérieurs stellaires ([16]), le gaz d'électrons obéit à la statistique de Maxwell-Boltzmann. Cependant, la statistique de Fermi-Dirac est la seule valable dans diverses situations, les électrons étant alors 'dégénérés'. Le degré de dégénérescence augmente avec l'énergie de Fermi du gaz, c-à-d avec une augmentation de la densité et/ou une baisse de la température 
est plus grande que l'énergie seuil de transitions endothermiques. L'évaluation de la vitesse de capture d'électrons libres peut reposer sur la connaissance expérimentale de la vitesse de la transition $\beta^{+}$inverse. Dans beaucoup de cas, cette information est cependant insuffisante et doit être complétée par des considérations théoriques. Parmi les transitions endothermiques les plus remarquables pouvant être rencontrées en astrophysique, citons la capture électronique par ${ }^{14} \mathrm{~N},{ }^{16} \mathrm{O},{ }^{20} \mathrm{Ne}$ ou ${ }^{24} \mathrm{Mg}$ dans certaines naines blanches $([46,47,48])$, ou la transformations de protons en neutrons dans le coeur à très haute densité d'une étoile massive sur le point d'exploser en supernova.

La capture de positons [Fig. 11(e)] peut également jouer un rôle important dans certaines situations stellaires, et particulièrement à haute température (typiquement $T>10^{9}$ $\mathrm{K}$ ) et faible densité. Dans ces conditions, une concentration assez élevée de positons peut résulter d'un équilibre $\mathrm{e}^{-}+\mathrm{e}^{+} \leftrightarrow \gamma+\gamma$.

4.4.2. Les réactions neutriniques Divers processus impliquant des neutrinos peuvent jouer un rôle important dans certains sites astrophysiques, et tout particulièrement dans les supernovae de Type II. Dans ces explosions, la probabilité de production des divers types de neutrinos ou d'antineutrinos au centre de l'étoile à neutrons en formation, leurs sections efficaces d'interaction avec la matière qui déterminent leur vitesse de transport jusqu'à la surface de l'étoile à neutrons, ainsi que l'interaction des neutrinos émergeant de l'étoile à neutrons avec les neutrons ou protons environnants constituent des ingrédients essentiels des modèles de supernova de Type II ([49]-[52]). Les neutrinos diffusant de l'étoile à neutrons pourraient également interagir avec des noyaux lourds pré-existants lors de leur passage dans des couches plus externes de l'étoile en explosion et conduire à une nucléosyntèse limitée appelée 'processus $\nu$ ' $([53,54])$.

Alors que la plupart des processus de désintégration $\beta$ d'intérêt astrophysique mentionnés plus haut peuvent être décrits dans le cadre de la théorie classique ' $\mathrm{V}-\mathrm{A}$ ' de l'interaction faible ([55]), l'évaluation des sections efficaces d'interaction neutrinique nécessite la prise en compte des courants chargés et neutres de l'interaction électro-faible ([56, 57]). L'interaction des neutrinos avec la matière pose bien d'autres questions aussi intéressantes que complexes qui ne peuvent être discutées ici (voir par exemple [58] - [60]).

\subsubsection{La désintégration $\beta$ de niveaux nucléaires excités Comme indiqué précédemment,} les niveaux excités et le fondamental d'un noyau ont souvent une population caractéristique de l'équilibre thermique, certains niveaux isomériques constituant des exceptions possibles. Ces divers niveaux peuvent donc contribuer à la désintégration $\beta$ d'un noyau, de sorte que sa demi-vie effective peut plus ou moins largement différer de la valeur du laboratoire.

Un exemple classique de l'importance de la désintégration $\beta$ de niveaux excités est fourni par ${ }^{99} \mathrm{Tc}$, qui est sans doute l'isotope du Technetium observé à la surface de certaines étoiles de type AGB. Cet isotope est produit par le processus s de nucléosynthèse (voir $\mathrm{S}$. Goriely, ce volume) à des températures excédant $10^{8} \mathrm{~K}$ environ. Dans de telles conditions, les niveaux de ${ }^{99} \mathrm{Tc}$ à 141 et $181 \mathrm{keV}$ peuvent être suffisamment peuplés pour contribuer de façon significative à la désintégration $\beta$. La Fig. 12 montre que la demi-vie effective de ${ }^{99} \mathrm{Tc}$ peut diminuer de façon spectaculaire de la valeur du laboratoire $\left(t_{1 / 2} \approx 2.1 \times 10^{5}\right.$ ans) à une valeur inférieure à 10 ans environ pour $T \gtrsim 3 \times 10^{8} \mathrm{~K}$. Cet exemple illustre clairement que la désintégration de niveaux excités peut jouer un rôle essentiel dans les conditions suivantes: (1) la désintégration $\beta$ de l'état fondamental est lente en raison des contraintes imposées

\# A noter également que la section efficace de capture de neutrinos par certains noyaux lourds, comme ${ }^{71} \mathrm{Ga}$ ou ${ }^{205} \mathrm{~Pb}$, est également nécessaire en vue de développer certains détecteurs à neutrinos 

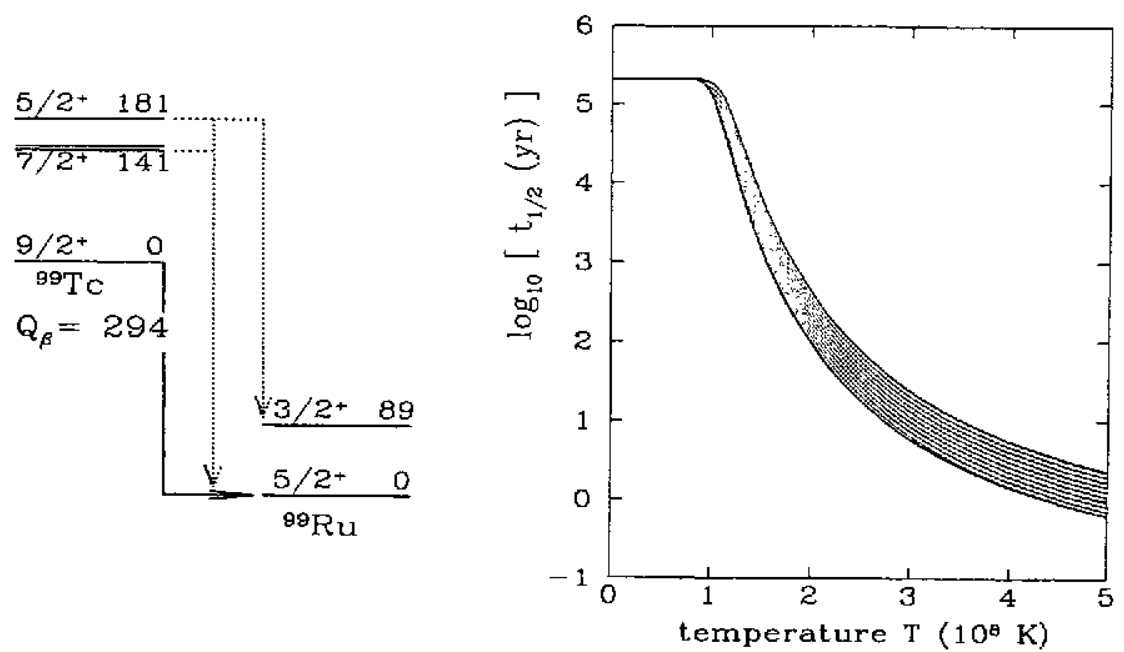

Figure 12. Figure de gauche: les états de basse énergie de ${ }^{99} \mathrm{Tc}$ et de son produit de désintégration $\left({ }^{99} \mathrm{Ru}\right.$ ) identifiés par leur énergie (en $\mathrm{keV}$ ) et leur spin-parité $J^{\pi}$. Le fondamental de ${ }^{99} \mathrm{Tc}$ se désintègre en laboratoire par une transition deux fois interdite très lente. La désintégration $\beta$ du niveau isomérique à $143 \mathrm{keV}$ (pas représenté) est également très lente. Au contraire, les niveaux à 141 et $181 \mathrm{keV}$ peuvent subir une transition permise (de type Gamow-Teller; flèches pointillées); Figure de droite: derni-vie $\beta$ effective de ${ }^{99} \mathrm{Tc}$ en fonction de la température si un équilibre de populations est atteint et sur base d'une estimation des éléments de matrice des transitions $\beta$ inconnus expérimentalement. La surface grisée représente les incertitudes probables sur la demi-vie effective

par les règles de sélection concernant les différences de spin et de parité entre état initial et final; (2) des niveaux excités à énergie relativement faible peuvent subir des transitions $\beta$ moins interdites que le fondamental vers des états du noyau fils. Les températures doivent évidemment être assez élevées pour assurer une population assez grande de ces niveaux excités.

\subsubsection{Les désintégrations $\beta$ à grande ionisation Comme souligné plus haut, l'ionisation des} atomes peut affecter de diverses façons les périodes de désintégration $\beta$. Les deux exemples qui suivent en fournissent une illustration.

La création e La prédiction théorique de ce processus date de plus de 50 ans [61], mais sa confirmation expérimentale n'a été fournie que très récemment $[62,63]$. En fait, l'importance de ce mécanisme dans la transformation de certains nucléides stables, comme ${ }^{163}$ Dy dans des intérieurs stellaires chauds avait déjà été soulignée au début des années 80 ([64]). L'intérêt pour ce processus en astrophysique n'a fait que croître depuis cette époque, notamment en relation avec le processus s et avec certaines études cosmochronologiques ([65]).

La désintégration $\beta$ de radionucléides dans le rayonnement cosmique Les abondances de. certains radionucléides dans le rayonnement cosmique peuvent servir à la détermination de l'âge de ces particules de haute énergieł† ou à une estimation du temps qui s'est écoulé entre la nucléosynthèse et l'accélération à des énergies typiques du rayonnement cosmique ([66]). Parmi les radionucléides intéressants, citons ${ }^{54} \mathrm{Mn}$ qui se désintègre par capture d'électrons orbitaux avec une demi-vie $t_{1 / 2}=312 \mathrm{j}$. A haute énergie, ce radionucléide est totalement ††Plus précisément, il s'agit du temps de confinement du rayonnement cosmique dans le disque de la Galaxie et dans son halo magnétique 
dépourvu de ses électrons orbitaux et ne peut se désintégrer que très lentement par processus $\beta^{ \pm}$(la demi-vie associée est très incertaine; [67]). Récemment [68], l'observation de ${ }^{59} \mathrm{Ni}$ dans le rayonnement cosmique (où il est également stabilisé) a permis de conclure que plus de $7.6 \times 10^{4}$ ans (la demi-vie de ${ }^{59} \mathrm{Ni}$ en laboratoire) se sont écoulés entre la production de ce radionucléide et son accélération dans le rayonnement cosmique. Il apparaît ainsi que le rayonnement cosmique ne peut être fait de matière fraîchement éjectée par une supernova, mais plutôt de matière interstellaire pré-existante. Un autre cas intéressant, en particulier pour l'astronomie $\gamma$-nucléaire, est fourni par ${ }^{44} \mathrm{Ti}$. Sa demi-vie qui est d'environ 60 ans en laboratoire ([69]), peut être allongée dans des éjecta de supernovae jeunes en raison de son ionisation [70].

\subsection{Désintégrations nucléaires et réactions via l'interaction électromagnétique}

Les noyaux immergés dans un bain de photons stellaires à haute température sont susceptibles de subir des photodésintégrations de type $(\gamma, \mathrm{n}),(\gamma, \mathrm{p})$ ou $(\gamma, \alpha)$. En raison des difficultés expérimentales (et théoriques) rencontrées dans la détermination directe des vitesses de photodésintégration, particulièrement lorsque les photons obéissent à une loi de distribution de Planckt, leur évaluation repose très généralement sur l'utilisation du 'théorème de la balance de détail' apliquée aux réactions inverses de capture de nucléons ou particules $\alpha$. Cette procédure met clairement en évidence la dépendance en $\exp (-Q / k T)$ des vitesses de photodésintégration, où $T$ est la température et $Q$ la 'valeur $Q$ ' de la réaction. Les photodésintégrations jouent donc un rôle de plus en plus important avec l'avancement de l'évolution des étoiles, c-à-d avec l'augmentation de température. En pratique, elles commencent à participer à l'énergétique et à la nucléosynthèse lors de la phase de 'combustion de $\mathrm{Ne}^{y}$ (Sects. 6.4.1,7.3,9.3), qui est en réalité une phase de photodésintégration dominée par ${ }^{20} \mathrm{Ne}(\gamma, \alpha){ }^{16} \mathrm{O}$. L'importance des photodésintégrations culminent lors de la phase supernova.

\subsection{Désintégrations nucléaires via l'interaction forte}

Les processus de fission, et en particulier celle induite par neutrons, peuvent déterminer la nature des nucléides les plus lourds susceptibles d'être synthétisés par le processus $r$ (voir S. Goriely, ce volume). Il n'est donc pas étonnant qu'ils aient attiré l'attention des astrophysiciens nucléaires, notammnent en relation avec la possibilité de production stellaire de noyaux super-lourds. D'autre part, la fission, spécialement de type $\beta$-retardée, présente l'intérêt supplémentaire d'être éventuellement responsable du recyclage vers des nucléides plus légers des noyaux les plus lourds produits dans le processus $\mathrm{r}$ (ce recyclage peut également être dû à des désintégrations $\alpha$ ). Enfin, l'énergie libérée par les fissions peut jouer un rôle non négligeable dans certains processus de captures rapides de neutrons, comme ceux qui pourraient être associés à des étoiles à neutrons ([71]).

En ce qui concerne les désintégrations $\alpha$, elles ont fait l'objet d'études extensives dans le laboratoire. Sauf dans quelques cas très particulier [72], les données expérimentales disponibles semblent suffire aux besoins astrophysiques. La situation est très différente en ce qui concerne la fission. En effet, les barrières de fission de noyaux très riches en neutrons impliqués dans le processus $\mathrm{r}$ ne peuvent être mesurées aujourd'hui dans le laboratoire et ne peuvent être évaluées que théoriquement.

† C'est la cas dans les intérieurs stellaires, où l'équilibre thermodynamique local constitue une très bonne approximation ([16]) 


\subsection{Les réactions thermonucléaires dans des événements astrophysiques non explosifs}

Aux données nucléaires d'intérêt astrophysique mentionnées dans les sections précédentes, il faut sans aucun doute ajouter la vitesse de réactions nucléaires dans des domaines d'énergie d'intérêt astrophysique. La détermination de ces vitesses a depuis bien longtemps été au centre d'une intense activité expérimentale et théorique. Des détails relatifs à ces recherches sont fournis par P. Descouvemont, S. Goriely et P. Leleux (ce volume). Nous nous limitons donc ici à quelques considérations et remarques très générales relatives aux réactions thermonucléaires en astrophysique. Les réactions de spallation (Sect. 3) ne seront pas discutées ici.

Les réactions thermonucléaires d'intérêt astrophysique concernent principalement la capture de nucléons ou particules $\alpha$. Un nombre limité de réactions impliquant des ions lourds légers $\left({ }^{12} \mathrm{C},{ }^{16} \mathrm{O}\right)$ sont également importantes. Comme souligné précédemment, les réactions induites par particules chargées contribuent de façon essentielle au budget énergétique d'une étoile, ainsi qu'à la production de nouveaux nucléides dans des situations non stellaires (Big Bang) ou stellaires. Au contraire, les captures de neutrons n'ont un impact qu'au niveau nucléosynthétique, leur contribution énergétique étant négligeable.

Dans des situations non explosives, correspondant en particulier aux phases 'calmes' de l'évolution stellaire qui se déroulent à température relativement basse, la plupart des réactions intéressantes impliquent des noyaux stables. Malgré cela, la détermination expérimentale de leur section efficace posent des problèmes d'une extrême complexité ([10]).

Le domaine d'énergie 'où presque rien ne se passe' Les difficultés expérimentales sont essentiellement dues au fait que les énergies d'intérêt astrophysique pour les réactions entre particules chargées sont beaucoup plus faibles que la barrière coulombienne. En conséquence, les sections efficaces peuvent plonger jusqu'à des valeurs de l'ordre du nanobarn au picobarn. Grâce à leur savoir-faire et d'héroiques efforts, les physiciens nucléaires impliqués dans l'astrophysique nucléaire ont été capables de fournir les sections efficaces les plus petites jamais mesurées en laboratoire, sans pouvoir cependant, sauf à l'une ou l'autre exception près, atteindre la région 'où presque rien ne se passe' d'intérêt astrophysique. L'aide théorique est donc indispensable en vue de fournir des extrapolations fiables des données expérimentales, ou même de prédire la vitesse de réactions n'ayant jamais été étudiées dans le laboratoire.

Corrections d'écran électronique De nouvelles difficultés sont venues s'ajouter à celles, déjà nombreuses, posées par la détermination des vitesses stellaires de réaction nucléaire avec la découverte, grâce à une série d'expériences remarquables, que les sections efficaces mesurées en laboratoire aux énergies les plus basses possibles peuvent être 'polluées' par des effets atomiques ou moléculaires induits par les conditions d'expérience elles-mêmes ([73] - [75]). En conséquence, la situation apparaît bien plus complexe qu'imaginée précédemment afin de passer des mesures en laboratoire aux vitesses stellaires: avant d'appliquer les corrections d'écran habituelles relatives aux plasmas stellaires ([76]), il faut tout d'abord extraire les effets d'écran du laboratoire des sections efficaces mesurées en vue de déduire les probabilités de réaction entre ions totalement ionisés constituant le plasma à l'intérieur des étoiles. En dépit de très nombreux efforts expérimentaux et théoriques, bien des incertitudes subsistent dans la mesure précise des effets d'écran dans les conditions du laboratoire. Des difficultés plus ou moins importantes subsistent également dans le calcul des effets d'écran stellaires. Cette situation peut être très gênante dans le traitement de certaines questions spécifiques, 
comme celle des neutrinos solaires (Sect. 1).

\subsection{Les réactions nucléaires dans des événements astrophysiques explosifs}

Dans des explosions stellaires (comme les novae et les supernovae) ou non-stellaires (Big Bang), les énergies d'intérêt astrophysique sont typiquement plus grandes que dans un environnement non-explosif (en raison des températures plus élevées associées aux explosions) et peuvent même atteindre des valeurs proches de la barrière coulombienne. Dans ces conditions, les sections efficaces de réaction sont également plus grandes, pouvant varier entre le micro- et le millibarn. Un prix élevé doit malheureusement être payé afin d'entrer dans ce domaine de sections efficaces.

Le monde de l'exotisme nucléaire Les particules $\alpha$, nucléons ou photons thermalisés dans un site astrophysique en explosion sont capables de pousser les flots nucléaires vers la région des noyaux très riches ou très déficients en neutrons. La description précise de ces flots nécessite donc la connaissance de la vitesse de capture de nucléons ou de particules $\alpha$ par des noyaux très instables dont la grande majorité n'a jamais été produite dans le laboratoire. Les vitesses de réaction sont donc exclusivement évaluées sur une base théorique.

L'excitation des noyaux cibles L'évaluation des vitesses de réaction se complique encore en raison du peuplement thermique d'un grand nombre de niveaux excités du noyau cible qui peuvent participer aux transmutations thermonucléaires. Cette contribution ne peut être évaluée que théoriquement, même si les noyaux cibles sont stables.

Une autre caractéristique des processus nucléaires opérant dans des situations explosives est la limitation drastique des durées de combustion, qui peuvent ne pas excéder des valeurs de l'ordre de la seconde à l'heure. En fait, une phase de combustion donnée peut dans certains cas se terminer bien avant l'épuisement des réactants, contrairement à la situation prévalant dans des conditions non explosives. Une telle situation se rencontre lorsque les échelles de temps de refroidissement de la matière (associées à sa vitesse d'expansion due à une explosion) devient plus courte que la vie moyenne d'un noyau pour la capture d'une particule chargée. Ces temps nucléaires caractéristiques augmentent en effet largement lorsque le milieu se refroidit en raison de la diminution des probabilités de transmission des barrières coulombiennes, liée elle-même à la diminution des énergies relatives moyennes des particules participant aux réactions (en raison de l'absence d'une telle barrière, la capture de neutrons n'est évidemment pas à prendre en compte dans cette discussion).

La nucléosynthèse explosive associée a très souvent été étudiée dans le cadre de modèles astrophysiques paramétrisés et souvent simplifiés à l'extrême. Une telle approche permet d'éviter les multiples difficultés rencontrées dans la prédiction des véritables caractéristiques de l'explosion, qui sont en fait très souvent inconnues. Au contraire, les flots nucléaires sont souvent décrits au moyen de réseaux détaillés (e.g. [11], Chap. 9). Cette approche hybride dans ses niveaux de sophistication astrophysique et nucléaire présente certainement quelques avantages. En particulier, elle permet de se faire une idée, qualitative du moins, des caractéristiques générales d'un processus nucléaire donné et même d'identifier quelques données nucléaires pouvant revêtir une importance particulière. Elle présente également d'évidentes déficiences et le danger que la validité des conclusions qui en sont tirées ne soient surévaluées sur les plans tant astrophysique que nucléaire. 


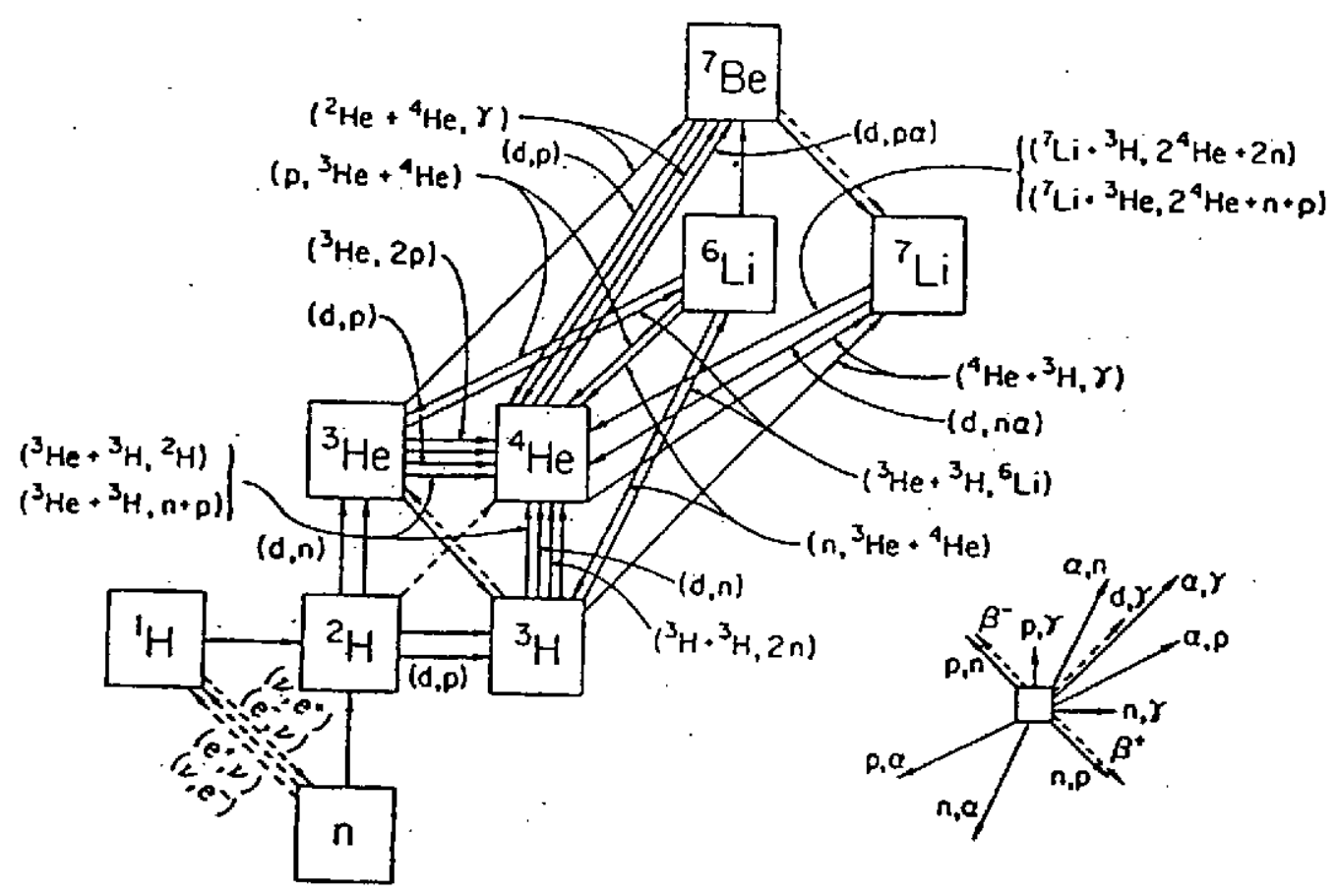
Figure 13. Le réseau des réactions nucléaires les plus importantes dans le cadre de la
NBBS

\section{Le Big Bang et la nucléosynthèse primordiale}

L'observation de l'expansion de l'Univers, du spectre de Planck de la radiation micro-onde à $3 \mathrm{~K}$, des fluctuations de densité dans la très faible anisotropie de ce rayonnement et dans la distribution locale des galaxies, ainsi que les prédictions relatives à la nucléosynthèse primordiale confirment aujourd'hui de façon spectaculaire la validité de la théorie du Big Bang et d'un Univers initialement chaud et dense.

En tant que l'un des piliers de la cosmologie moderne, la nucléosynthèse accompagnant le Big Bang a fait l'objet de myriades de travaux dont il est impossible de rendre compte dans le cadre de ces leçons. Des détails concernant les modèles de Big Bang et leurs multiples ramifications en physique des particules, en physique nucléaire et en astrophysique observationnelle ou théorique peuvent être trouvés dans un certain nombre de revues récentes ([95] - [98]). Nous nous limiterons ici à quelques considérations très générales. Il convient tout d'abord de souligner que la nucléosynthèse accompagnant le Big Bang (NBB) se produit aux premiers instants dans l'histoire de l'Univers pour lesquels les processus physiques sont bien compris. Des prédictions détaillées peuvent dès lors être faites et confrontées à une grande variété de données observationnelles. En particulier, le modèle de Big Bang dit 'standard' (BBS) fournit une description particulièrement 'économique' (en terme de nombre de paramètres libres) de l'Univers observable. En fait, et une fois connue la température actuelle $T_{0}$ du fond micro-onde (approximativement $2.73 \mathrm{~K}$ ), le modèle BBS ne contient qu'un seul paramètre cosmologique, à savoir le rapport $\eta \equiv n_{b} / n_{\gamma}$ du nombre de baryons à celui des photons.

La Fig. 14 illustre clairement les caractéristiques essentielles de la NBBS calculée sur base du réseau de réactions représenté à la Fig. 13, à savoir une production plus ou moins importante à des tempratures voisines de $10^{9} \mathrm{~K}$ des nucléides légers $\mathrm{D},{ }^{3} \mathrm{He},{ }^{4} \mathrm{He},{ }^{7} \mathrm{Li}$ au 


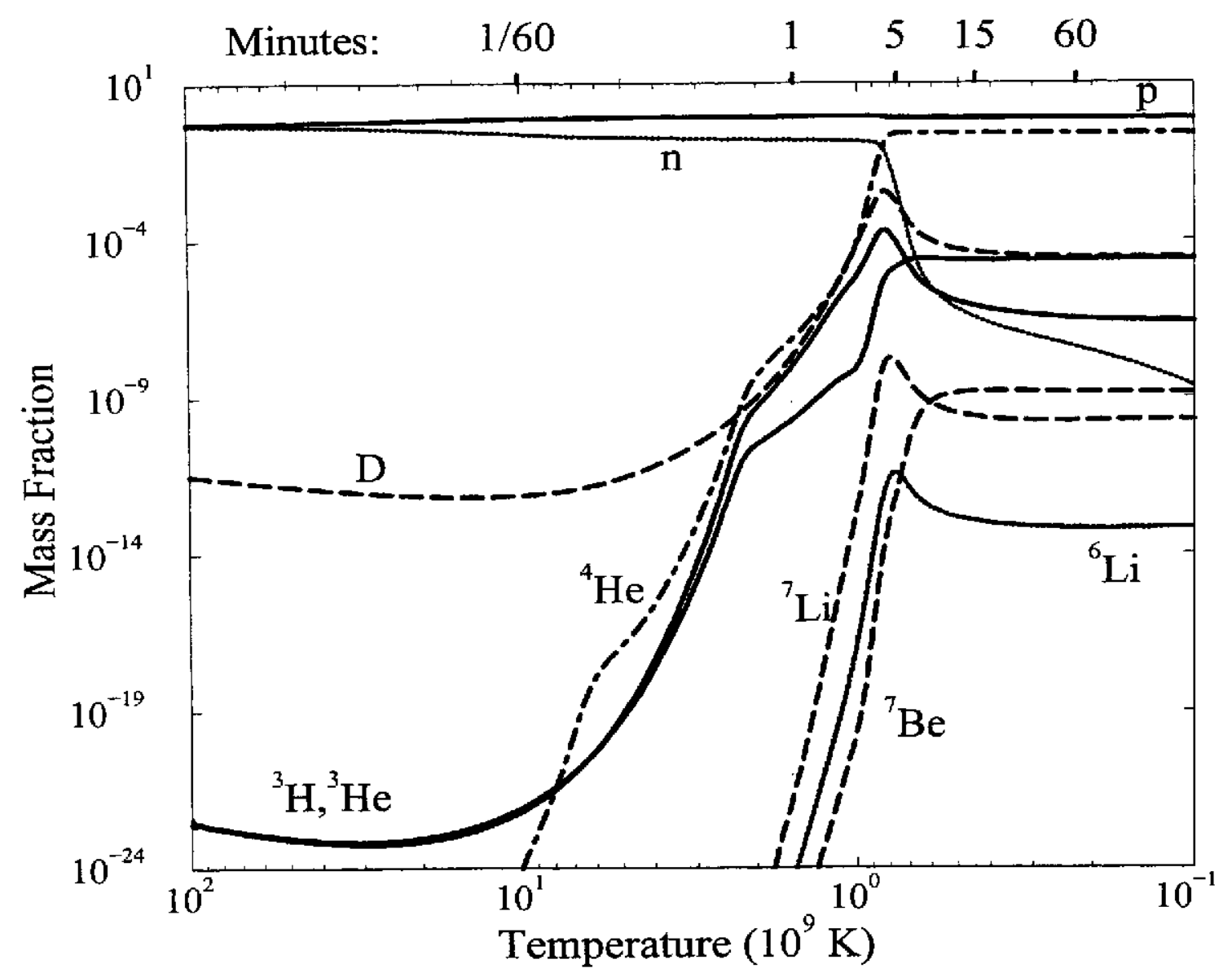

Figure 14. Fraction de masse des nucléides produits dans le BBS en fonction du temps écoulé lors de l'expansion de l'Univers (abscisse supérieure) ou, de façon équivalente, de la température (abscisse inférieure), la valeur adoptée du paramètre $\eta$ étant égale à $5.1 \times 10^{-10}$ (figure tirée de [98])

dépend des neutrons et protons initiaux. Le nucléide le plus abondamment produit est ${ }^{4} \mathrm{He}$. Cette situation résulte de la difficulté qu'a le flot nucléaire à dépasser le nombre de masse $A=5$ pour lequel n'existe aucun noyau stable. Il faut noter que l'abondance de ${ }^{4} \mathrm{He}$ calculée dans le cadre du BBS dépend essentiellement de la demi-vie du neutron et du nombre $N_{\nu}$ d'espèces de neutrinos légers. Ainsi, la NBBS a fourni durant les années 80 la contrainte la plus forte sur $N_{\nu}$, spectaculairement confirmée ensuite en laboratoire $\left(N_{\nu}\right.$ compatible avec 3). L'absence de noyau stable à $A=8$ joue également un rôle clé dans la NBBS. Cette situation impose en effet une production très faible de nucléides à $A>7$ (ces conclusions sont largement indépendantes de tout choix de la valeur de $\eta$ pouvant être compatible avec le modèle BBS). La Fig. 15 complète les informations nucléosynthétiques fournies par la Fig. 14 en présentant les abondances finales obtenues dans le cadre du $\mathrm{BBS}$ en fonction de $\eta$ (comme illustré à la Fig. 14, l'abondance des nucléides stables reste constante lorque la température devient insuffisante pour permettre aux réactions nucléaires entre particules chargées d'opérer assez rapidement par rapport au temps d'expansion de l'Univers (Sect. 4.8)). Cette figure illustre également l'impact des incertitudes nucléaires sur les prédictions de la NBBS au travers de l'utilisation des valeurs des vitesses de réaction nucléaire 'adoptées' et de leurs limites inférieure et supérieure fournies par la compilation NACRE [78]. Parmi les incertitudes nucléaires les plus importantes pouvant être identifiées 

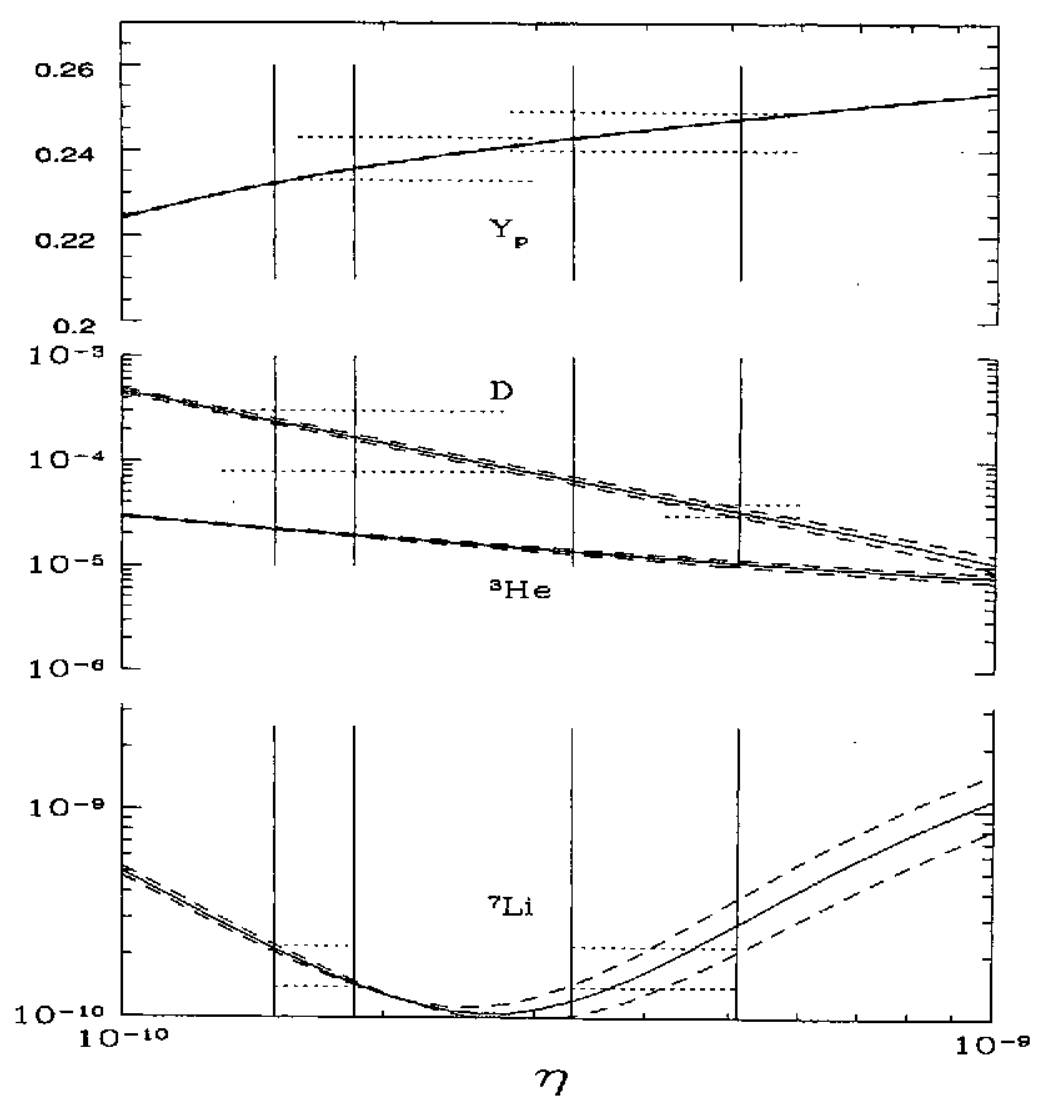

Figure 15. Prédictions nucléosynthétiques dans le cadre du modèle standard du Big Bang (NBBS). Les abondances de ${ }^{4} \mathrm{He}$ (exprimées en fraction de masse $\mathrm{Y}_{\mathrm{p}}$ ) et de $\mathrm{D},{ }^{3} \mathrm{He}$ et ${ }^{7} \mathrm{Li}$ (exprimées en densité numérique) sont représentées en fonction de $\eta$. Les lignes en trait plein sont obtenues en utilisant les vitesses de réaction 'adoptées' fournies par la compilation NACRE [78]. Les lignes pointillées résultent de l'utilisation des limites supérieure et inférieure des vitesses proposées par NACRE. Les lignes horizontales fournissent les limites déduites de diverses observations: ${ }^{4} \mathrm{He}$ à gauche: [100]; ${ }^{4} \mathrm{He}$ à droite: [101]; D à gauche: [102]; D à droite: [98]; ${ }^{7} \mathrm{Li}[103,104]$. Les lignes verticales correspondent aux limites de $\eta$ compatibles avec les données observationnelles relatives à ${ }^{7} \mathrm{Li}$ (figure tirée de [99])

à partir de ces calculs, citons $\mathrm{D}(\mathrm{p}, \gamma)^{3} \mathrm{He}$ qui intervient à assez haute valeur de $\eta$ au travers de ${ }^{3} \mathrm{He}(\alpha, \gamma)^{7} \mathrm{Be}(e, \nu)^{7} \mathrm{Li}$.

A ce point, il convient de confronter les prédictions de la NBBS aux observations. Cette comparaison soulève de multiples difficultés et est entachée de nombreuses incertitudes non seulement au niveau des abondances prédites, mais également en ce qui concerne les données pouvant être fournies par l'observation. En ce domaine, l'un des principaux problèmes concerne le fait que la comparaison avec la théorie doit se faire sur base d'abondances 'primordiales' qui ne peuvent être mesurées directement, mais qui, au contraire, doivent être obtenues sur base d'observations extrapolées à l'ère pré-galactique grâce à un modèle d'évolution chimique de la Galaxie ou des galaxies. Il sort du cadre de ces leçons de mener une discussion détallée de la détermination de ces abondances primordiales. Le lecteur intéressé peut consulter utilement ([95] - [98]). La Fig. 15 fournit les limites inférieure et supérieure des abondances primordiales de $\mathrm{D},{ }^{4} \mathrm{He}$ et ${ }^{7} \mathrm{Li}$ sélectionnées par [99]. Il faut noter que l'abondance primordiale de ${ }^{3} \mathrm{He}$ n'est pas représentée en raison des très grandes incertitudes affectant sa valeur. Il convient également de signaler que deux domaines disjoints de valeurs de 
l'abondance primordiale de D proposés par deux groupes d'observateurs apparaissent à la Fig. 15. Cette situation illustre particulièrement clairement l'ampleur des problèmes posés par la détermination de certaines abondances primordiales.

La Fig. 15 montre que les abondances de ${ }^{7} \mathrm{Li}$ prédites par la NBBS et celles déduites de l'observation peuvent être réconciliées pour des valeurs de $\eta$ comprises dans les domaines $1.4<\eta_{10}<1.9$ ou $3.3<\eta_{10}<5.1\left(\eta=10^{-10} \times \eta_{10}\right)$. En termes du rapport $\Omega_{0, B} \equiv \rho_{B, 0} / \rho_{c, 0}$ de la densité baryonique actuelle à la densité critique actuelle assurant la fermeture de l'Univers, ces limites sur $\eta$ peuvent se traduire par $0.013<\Omega_{B, 0}<0.017$ ou $0.017<\Omega_{B, 0}<0.045$ sur base de la relation $\Omega_{B, 0} \approx 0.0036 h_{0}^{-2} \eta_{10}\left(T_{0} / 2.73\right)^{3}$, où $h_{0}$, défini à partir de la constante de Hubble actuelle $H_{0}$ par $H_{0} \equiv 100 h_{0} \mathrm{kms}^{-1} \mathrm{Mpc}^{-1}(1 \mathrm{Mpc}$ $=1$ Mégaparsec), est adopté égal à 0.65 , cette valeur (encore affectée d'incertitudes) étant déduite d'observations récentes. L'interprétation de ces valeurs baryoniques dans le cadre d'un modèle de la matière noire est un sujet d'un grand intérêt cosmologique qui ne peut être abordé ici.

Les deux domaines de $\eta$ (ou $\Omega_{B, 0}$ ) qui viennent d'être définis, ou du moins une partie de ces domaines, permettent également de rendre compte de l'abondance de $\mathrm{D}$ et de ${ }^{4} \mathrm{He}$. Plus précisément, le premier domaine fournit une explication pour les abondances de D élevée et de ${ }^{4} \mathrm{He}$ faible, le second domaine rendant au contraire compte des abondances de $\mathrm{D}$ faible et de ${ }^{4} \mathrm{He}$ élevée. Les incertitudes actuelles ne permettent pas de préférer l'une des solutions à l'autre. A cet égard, une détermination plus précise de l'abondance primordiale de $\mathrm{D}$ serait essentielle. Il faut également noter que les domaines de valeurs de $\eta$ (ou $\Omega_{B, 0}$ ) capables de rendre compte des abondances primordiales de $\mathrm{D},{ }^{4} \mathrm{He}$ et ${ }^{7} \mathrm{Li}$ peuvent varier d'un auteur à l'autre, dépendant de leur sélection des abondances primordiales 'observées' et des prédictions théoriques. Il n'en reste pas moins que la capacité du BBS de rendre compte des abondances de trois nucléides au moins (le cas de ${ }^{3} \mathrm{He}$ est incertain) à l'aide d'un seul paramètre libre est généralement considéré comme l'un des triomphes de ce modèle.

Remarquons enfin que de nombreux modèles 'non-standards' de BB ont également été développés. En particulier, l'un de ceux-ci repose sur l'existence d'inhomogénéités à l'époque de la nucléosynthèse et a soulevé un grand intérêt dans la communauté des astrophysiciens nucléaires $([105,106])$. Ce type de modèles fait en effet intervenir beaucoup plus de réactions que le BBS (Fig. 13), beaucoup de ces transformations ayant des vitesses inconnues dans la mesure où elles font intervenir des noyaux instables. Il est évident que l'intérêt de poursuivre des recherches expérimentales ou théoriques au sujet de ces réactions dépend avant tout de la validité de ces modèles inhomogènes, qui est loin d'être démontrée aujourd'hui $([95,107])$.

\section{Structure et évolution stellaire en bref}

Dans le cadre de ces leçons, il est impossible de mener une étude approfondie de la structure et de l'évolution des étoiles, dont la connaissance est essentielle dans de nombreux domaines de l'astrophysique, et en particulier dans l'interprétation du DHR (Sect. 2.1), de la composition de surface de certaines étoiles chimiquement particulières (Sect. 2.2.3) ou dans la construction de modèles d'évolution chimique de la Galaxie (Sect. 3). Nous nous contenterons ici de fournir quelques informations de base et de formuler quelques remarques générales. Le lecteur intéressé peut consulter $([11,16,108])$ pour obtenir de plus amples informations. 


\subsection{Etoiles à l'équilibre mécanique et énergétique}

Sous les hypothèses d'une étoile gazeuse non magnétisée et qui n'est animée d'aucun mouvement de rotation, l'équibre mécanique se définit comme un état stationnaire caractérisé par l'égalité de la force de gravitation (dirigée vers le centre de l'étoile) et de la force associée à la pression exercée par la radiation et par le gaz (dirigée vers l'extérieur de l'étoile), cet état stationnaire étant réalisé sur des échelles de temps longues par rapport au temps de chute libre. A titre indicatif, ce dernier est de l'ordre de l'heure pour le Soleil. Cette condition d'équilibre mécanique se traduit par une équation qui prend une forme particulièrement simple sous l'hypothèse d'une configuration stellaire à symétrie sphérique.

D'autre part, l'équilibre énergétique s'interprète comme un état stationnaire pour lequel l'énergie produite par les transmutations nucléaires est capable de compenser en tout point d'une étoile l'énergie perdue par radiation, mouvements convectifs, neutrinos, conduction, ainsi que par 'vents stellaires'. L'état stationnaire ainsi défini doit être réalisé sur des durées longues par rapport au 'temps de Helmholtz-Kelvin', correspondant au temps nécessaire pour que la puissance émise par une étoile ('luminosité') s'annule en l'absence de sources d'énergie nucléaire. Pour le Soleil, ce temps est typiquement de l'ordre d'une dizaine de millions d'années, et est donc beaucoup plus long que le temps de chute libre.

Les conditions d'équilibre mécanique et énergétique fournissent deux équations. Elles sont complétées par l'équation de continuité et par une équation exprimant le gradient de température en chaque point de l'étoile en fonction du mode de transport de l'énergie (radiation, convection, conduction,...). Ainsi, la construction d'un modèle d'étoile à l'équilibre mécanique et énergétique se ramène à la résolution d'un système de quatre équations différentielles non linéaires du premier ordre. Si l'existence d'une solution peut être démontrée, il n'en va pas de même de son unicité. Sous certaines conditions restrictives, une proposition peut toutefois être énoncée suggérant que l'unicité est susceptible de caractériser une très large classe de modèles stellaires. Cette proposition est appelée 'théorème de VogtRussell' dans la littérature astrophysique. Il ne s'agit pourtant nullement d'un théorème au sens mathématique dans la mesure où il ne peut être démontré de façon rigoureuse (en fait, des cas violant ce 'théorème' ont été identifiés), mais doit plutôt être considéré comme un 'outil de travail' d'une grande utilité dans l'interprétation de nombreuses observations stellaires ([16] pour une discussion détaillée des brèves considérations qui précèdent). Ainsi, le DHR peut être vu comme l'une des manifestations les plus spectaculaires du théorème de Vogt-Russell, les lignes de corrélation observées (voir Figs. 1 et 2) étant interprétées dans ce cadre comme la signature des équilibres mécanique et énergétique pouvant être réalisés par des étoiles de masse et de composition initiale données pour des ensembles bien spécifiques de température effective et de luminosité. En vertu de la définition de l'équilibre énergétique fournie ci-dessus, les lignes de corrélation du DHR peuvent également être vues comme un témoignage de la capacité qu'ont les transmutations nucléaires à couvrir les pertes énergétiques de certaines classes d'étoiles.

\subsection{Etoiles hors d'équilibre énergétique: comment répondent-elles?}

L'équilibre énergétique d'une étoile n'est pas assuré si la production d'énergie nucléaire est soit trop petite, soit trop grande par rapport aux pertes d'énergie sur des temps longs par rapport au temps de Helmholtz-Kelvin. Nous discutons de la réponse d'une étoile à ces différentes situations au travers d'une analyse grossière reposant sur les schémas présentés aux Figs. $16-18$.

Ainsi qu'indiqué à la Fig. 16, toute la discussion se fait sur base d'une équation 


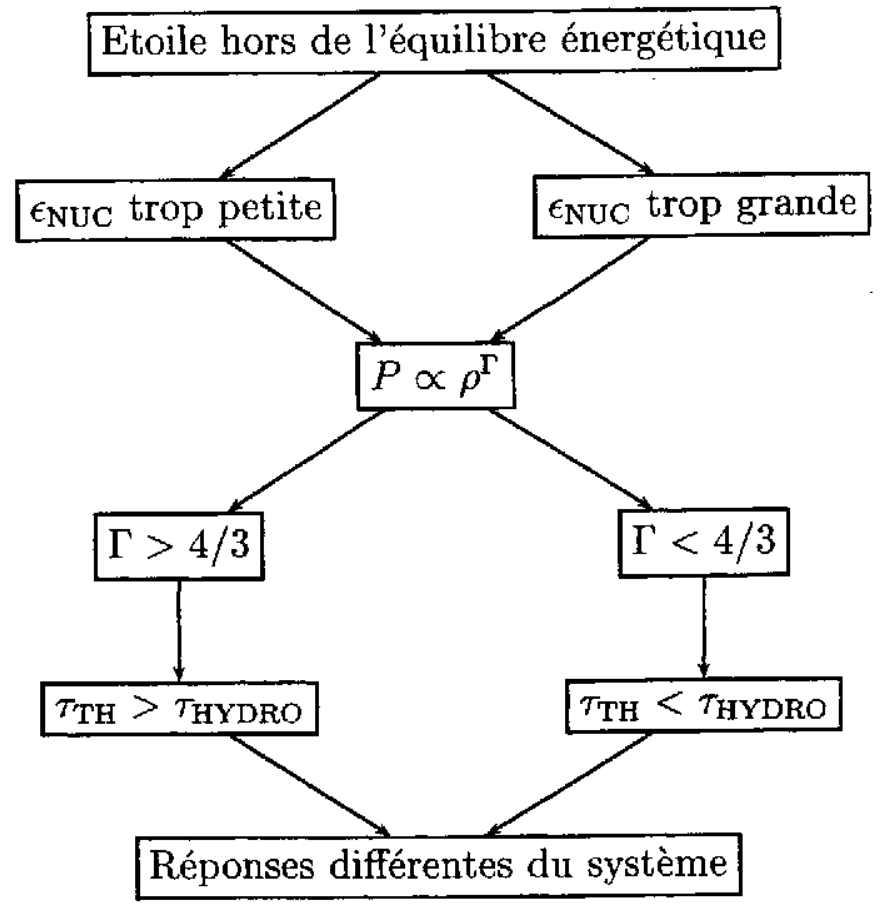

Figure 16. Représentation schématique des situations conduisant à un écart d'une êtoile à l'équilibre énergétique. Une équation d'état de type polytropique est adoptée. Les différents symboles sont définis dans le texte

d'état simple de type polytropique $P \propto \rho^{\Gamma}$, où $P$ est la pression, $\rho$ la densité et $\Gamma$ un 'exposant polytropique'. Dans ce cas, la réponse d'une étoile à un excès (Fig. 18) ou à un déficit (Fig. 17) de production d'énergie nucléaire (représentée par $\varepsilon_{\mathrm{NUC}}$, qui est l'énergie nucléaire produite par unité de temps et de masse) est très différente suivant que $\Gamma$ est supérieur ou inférieur à $4 / 3$ dans une fraction suffisante de la masse de l'étoile (voir [16] pour une discussion). Le premier cas conduit à l'inégalité $\tau_{\mathbf{T H}}>\tau_{\text {HYDRO}}$. Dans cette relation, $\tau_{\mathrm{TH}} \propto 1 / \epsilon_{\mathrm{NUC}}$ peut s'interpréter comme un temps caractéristique d'allumage des réactions nucléaires fournissant une mesure de la vitesse d'augmentation de la température $T(t) \propto \exp \left(t / \tau_{\mathrm{TH}}\right)$ suite à la production d'énergie nucléaire. D'autre part, $\tau_{\mathrm{HYDRO}}$ est un temps caractéristique de réponse de l'étoile à un écart à l'équilibre mécanique et peut s'exprimer par $\tau_{\text {HYDRO }} \propto \delta r / C_{s}$, où $\delta r$ est une petite perturbation par rapport à la position d'équilibre mécanique, tandis que $C_{s}$ est la valeur locale de la vitesse du son. Au contraire, $\tau_{\text {TH }}<\tau_{\text {HYDRo }}$ si $\Gamma<4 / 3$. Les Figs. 17 et 18 fournissent quelques détails sur la réponse d'une étoile à ces diverses situations.

La Fig. 17 indique sous forme schématique la réponse possible d'une étoile à un écart à l'équilibre énergétique dû à une production d'énergie nucléaire insuffisante pour couvrir les pertes. Si l'équation d'état est de la forme $P \propto \rho^{\Gamma>4 / 3}$ (ce qui est équivalent à $\tau_{\mathrm{TH}}>\tau_{\text {HYDRO}}$; voir Fig. 16), l'étoile subit une contraction 'quasi-hydrostatique'. Cette situation se rapporte à une contraction lente par rapport au temps de chute libre, ce qui se traduit par $\tau_{\mathrm{CONTR}} \gg \tau_{\mathrm{CL}}$ où $\tau_{\mathrm{CONTR}}$ définit l'échelle de temps de la contraction, tandis que $\tau_{\mathrm{CL}}$ est le temps de chute libre $\left[\tau_{\mathrm{CL}} \sim 2\left(R^{3} / G M\right)^{1 / 2}\right.$, où $R$ et $M$ sont respectivement le rayon et la masse de l'étoile considérée, $G$ étant la constante de la gravitation]. Cette contraction relativement lente permet à l'étoile de couvrir ses pertes énergétiques grâce à l'énergie puisée dans le puits gravitationnel (voir Fig. 17). Elle conduit également à une augmentation de température et de densité, du moins si le gaz n'est pas dégénéré. Ces augmentations peuvent 


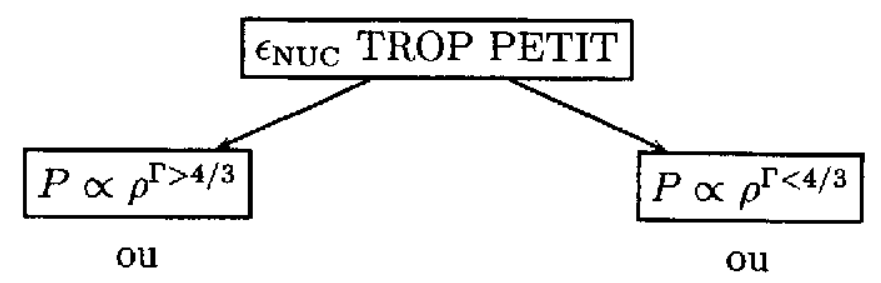

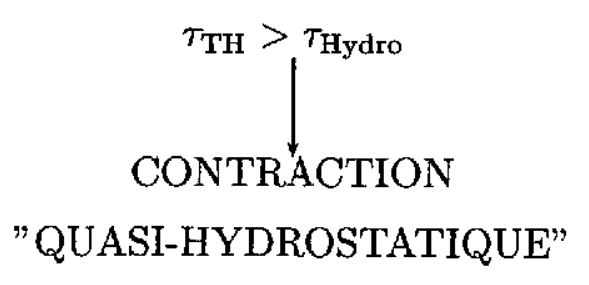

$\tau_{\mathrm{CONTR}} \gg \tau_{\mathrm{CL}}$

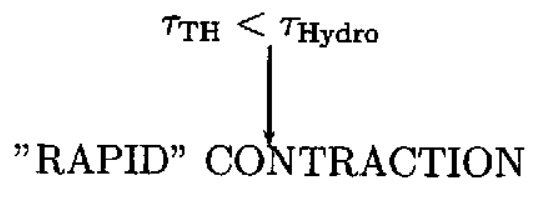

$=$ "COLLAPSE"

$$
\tau_{\mathrm{CONTR}} \approx \tau_{\mathrm{CL}}
$$

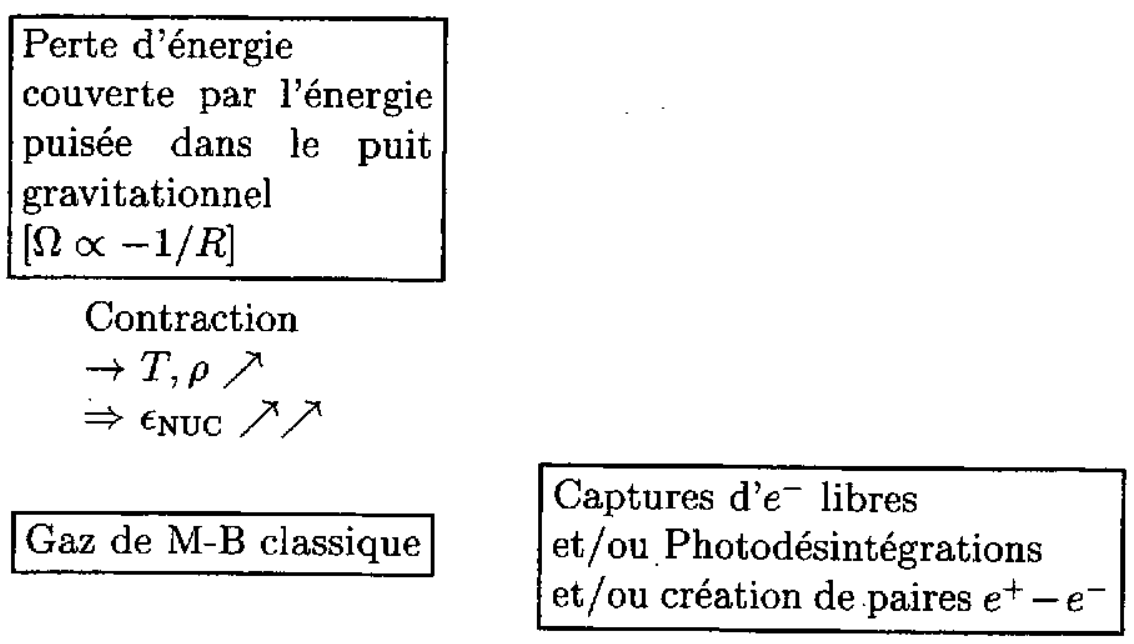

Figure 17. Diagramme schérnatique de la réponse d'une étoile 'a un écart à l'équilibre énergétique dû à une production d'énergie nucléaire trop faible pour couvrir les pertes (voir texte)

aider à accroître $\epsilon_{\mathrm{NUC}}$, et donc à rétablir l'équilibre énergétique.

La réponse de l'étoile est différente si $P \propto \rho^{\Gamma<4 / 3}$ (ou $\tau_{\mathrm{TH}}<\tau_{\mathrm{HYDRO}}$; voir Fig. 16). Dans ce cas, la contraction se fait sur des échelles de temps de l'ordre du temps de chute libre $\left(\tau_{\mathrm{CONTR}} \sim \tau_{\mathrm{CL}}\right)$ et s'assimile à un 'effondrement'. Cette situation se rencontre notamment dans le cas de très fréquentes captures d'électrons libres ou de photodésintégrations (endothermiques), ainsi qu'en présence de nombreuses créations de paires $e^{+}-e^{-}$(c-à-d à hautes températures et faibles densités). Ces implosions conduisent en général à une phase 'destructive' dans l'évolution d'une étoile.

La Fig. 18 est le pendant de la Fig. 17 dans le cas où l'équilibre énergétique ne peut plus être assuré en raison d'une production d'énergie trop grande par rapport aux pertes que peut subir l'étoile. Dans ce cas, les deux situations considérées à la Fig. 17 $(\Gamma>4 / 3$ ou $\Gamma<4 / 3)$ doivent à nouveau être prises en compte et conduisent à des scénarios très différents. Dans le cas $\Gamma>4 / 3$, la situation est symétrique à celle décrite à la Fig. 17. Une expansion 'quasi-hydrostatique' se produit, caractérisée par un temps d'expansion $\tau_{\mathrm{EXP}} \gg \tau_{\mathrm{EXL}}$, où $\tau_{\mathrm{EXL}} \propto \rho^{-1 / 2}$ est le 'temps d'expansion libre'. L'injection d'énergie dans le puits gravitationnel afin de produire cette expansion est responsable d'un 

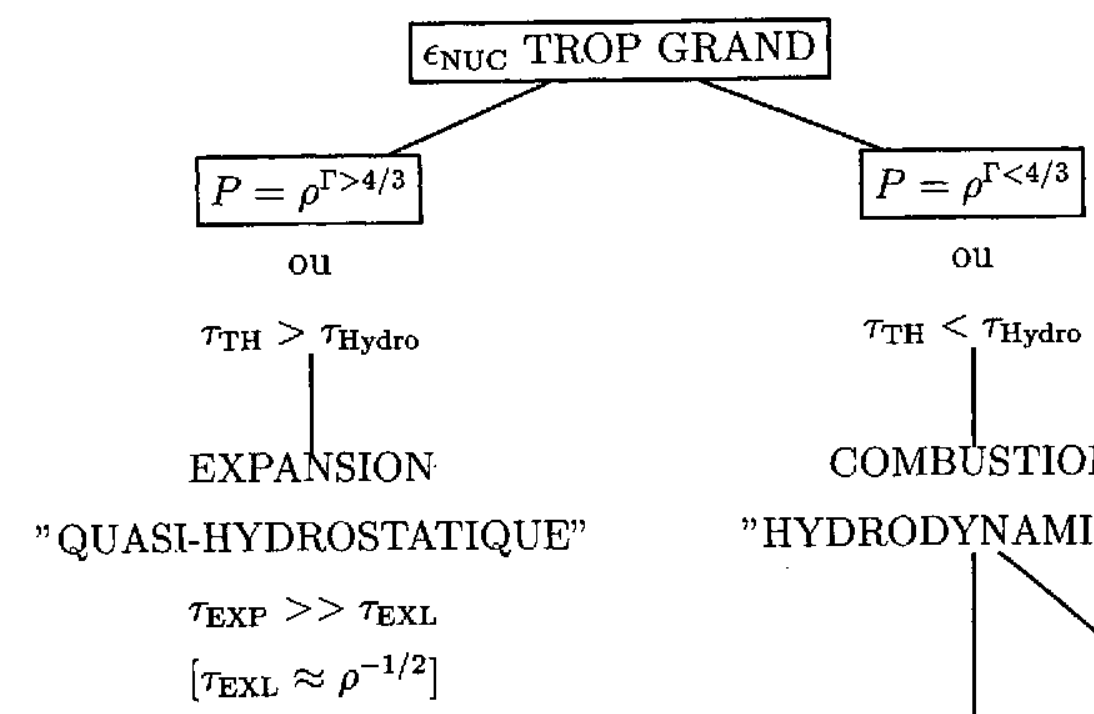

\begin{tabular}{|l|}
\hline $\begin{array}{l}\text { Excès d'énergie } \\
\text { absorbé dans le puits } \\
\text { gravitationnel } \\
{[\Omega \propto-1 / R]}\end{array}$ \\
\hline
\end{tabular}

Expansion

$\rightarrow T, \rho \searrow$

$\Rightarrow \epsilon_{\mathrm{NUC}} \searrow \searrow$

Gaz de M-B classique ou

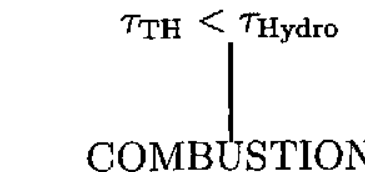

"HYDRODYNAMIQUE"

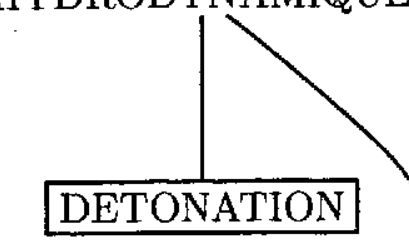

Figure 18. Diagramme schématique de la réponse d'une étoile à un écart à l'équilibre énergétique dû à une production d'énergie nucléaire trop importante par rapport aux pertes que peut subir l'étoile (voir texte)

refroidissement et d'une baisse de la densité, ce qui a tendance à diminuer $\epsilon_{\mathrm{Nuc}}$ et donc à éventuellement restaurer l'équilibre énergétique. Cette situation stable se rencontre dans les gaz non dégénérés.

Au contraire, si $\Gamma<4 / 3$, un régime de combustion hydrodynamique de type 'détonation' ou 'déflagration' s'installe, pouvant conduire à une phase destructive dans l'évolution d'une étoile. De telles situations se rencontrent notamment dans le cas de gaz très dégénérés. La physique des combustions hydrodynamiques est d'une très grande complexité, en particulier dans le cas stellaire, et est loin encore d'être comprise. Dans ces leçons, nous nous limiterons à quelques considérations de base pouvant intéresser l'astrophysicien nucléaire.

\subsection{Quelques généralités sur les combustions hydrodynamiques}

D'une façon générale, toute combustion hydrodynamique peut être vue comme un processus se déroulant en deux étapes. La première (Phase $\mathrm{I}$ ) est un 'emballement thermique' dans le 'volume de réaction'. La condition $\tau_{\mathrm{TH}}<\tau_{\mathrm{HYDRO}}$ sous laquelle peut se produire une combustion hydrodynamique exprime que le temps caractéristique d'augmentation 


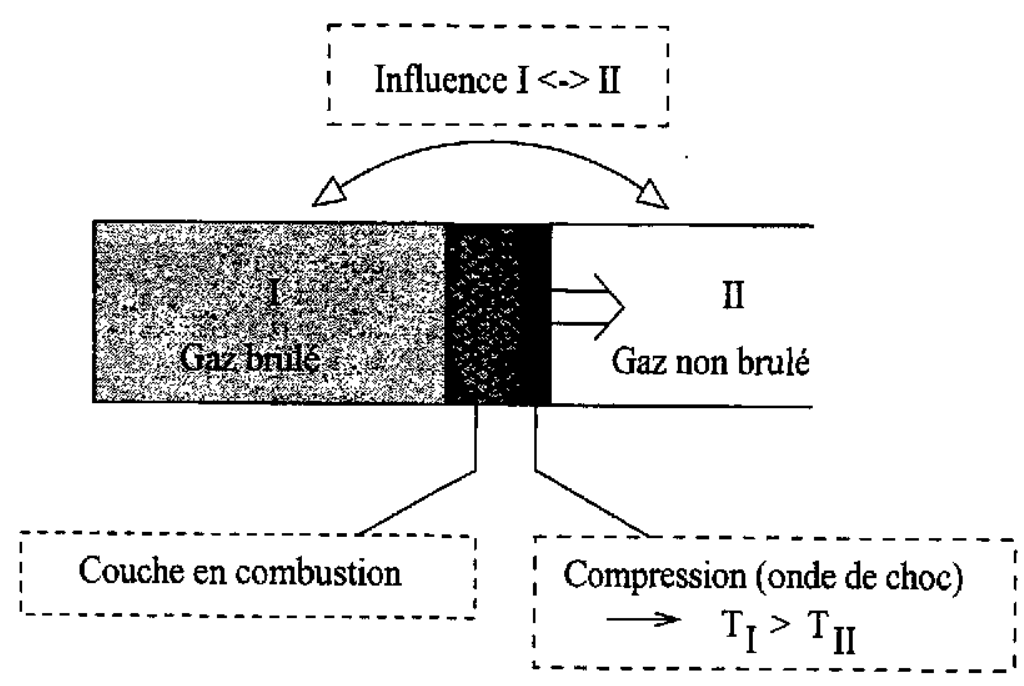

Figure 19. Représentation très schématique d'un régime de détonation, caractérisé par la propagation supersonique d'une onde de choc associée à une couche de combustion

de température suite à la production d'énergie nucléaire est plus court que le temps de propagation des ondes de pressions (ou ondes acoustiques). Il en résulte une surchauffe et une surpression dans le volume de réaction. La seconde étape du processus (Phase II) se rapporte à la propagation de 'l'onde de combustion'. La surpression résultant de la Phase I atteint les limites du volume de réaction initial et finit par se déplacer au-delà de ces limites. La surchauffe et la combustion nucléaire peuvent dès lors se produire dans un volume de réaction plus étendu que le volume initial, cette situation se développant en un phénomène explosif de type détonation ou déflagration. Il est important de noter que cette combustion explosive ne peut être provoquée par la Phase II seulement, mais résulte de la succession des deux étapes brièvement définies ci-dessus.

6.3.1. Les détonations en très bref Un régime de détonation peut se développer si la Phase I conduit à une surpression suffisamment importante pour engendrer une onde de choc à l'interface de la couche de combustion et du gaz non brûlé plus froid (Fig. 19). L'onde de choc entretient le brûlage nucléaire et donc la couche de combustion. L'ensemble constitué par la couche de combustion et par l'onde de choc est appelé 'l'onde de détonation'. Celleci se propage supersoniquement par rapport au gaz non brûlé. Dans les modèles les plus simples, l'onde de détonation est supposée avoir une épaisseur inférieure à quelques libres parcours moyens des particules constitutives du gaz. En réalité, la situation est clairement beaucoup plus compliquée.

6.3.2. Les déflagrations en très bref Le régime de déflagration se distingue essentiellement de celui d'une détonation par l'absence du développement d'une onde de choc en raison d'une surpression trop faible lors de la Phase I. Dans ces conditions, l'onde de combustion doit se propager 'pas à pas' par diffusion de matière (le gaz brûlé et chaud se mélangeant au gaz non brûlé) et par diffusion thermique (un flux de chaleur se créant entre le gaz brûlé et celui non brûlé). L'onde de déflagration peut ainsi être interprétée comme un front de flamme, l'image qualitative en étant un 'feu de forêt' se propageant de proche en proche. Cette propogation est subsonique par rapport au gaz non brûlé, contrairement à la situation rencontrée dans une détonation. Il en résulte notamment que les ondes de pression (acoustiques) peuvent se 
propager plus vite que le front de flamme, de sorte qu'un choc peut prendre naissance dans le gaz non brûlé, ce choc pouvant être trop faible ou assez fort pour donner naissance à une détonation. On parle dans ce dernier cas d'une détonation retardée.

La modélisation d'un régime de déflagration dans des conditions astrophysiques est d'une extrême complexité. Parmi les problèmes non résolus, il convient de citer la vitesse de propagation, la géométrie (sans doute très compliquée) du front de flamme, ainsi que les conditions physiques auxquelles s'opère la transition de la déflagration à la détonation dans un régime de détonation retardée. Ces caractéristiques sont intimement liées et influencent largement les transmutations nucléaires suscesptibles d'accompagner une déflagration. Tout progrès dans la description des déflagrations est donc d'un intérêt tout particulier pour l'astrophysique nucléaire.

\subsection{Comment une étoile évolue-t-elle?}

En bref, et comme décrit très schématiquement à la Fig. 20, l'évolution 'normale' (nonexplosive) des régions centrales d'une étoile est faite d'une succession de phases d'équilibre mécanique et énergétique et de phases de quasi-équilibre hydrostatique (voir Fig. 17). L'équilibre énergétique est assuré par des combustions nucléaires entre particules chargées qui sont responsables d'une modification de la composition du coeur de l'étoile sur des échelles de temps longues par rapport au temps de Helmholtz-Kelvin $t_{\mathrm{HK}}$ (Sect. 6.1). Dans ces conditions, l'évolution d'une étoile lors d'une phase de combustion nucléaire n'est qu'un déplacement d'un état d'équilibre mécanique et énergétique à un autre, la variable gouvernant ce déplacement étant la composition chimique. Il faut noter que ces variations de composition sont dues non seulement aux réactions assurant l'équilibre énergétique, mais également, dans une moindre mesure toutefois, à des captures de neutrons qui, si elles jouent un rôle nucléosynthétique important, ne contribuent pas de façon significative au bilan énergétique de l'étoile. Les phases de quasi-équilibre hydrostatique sont responsables d'une augmentation de température du coeur stellaire dues aux contractions gravitationnelles associées. Dans l'étoile considérée à la Fig. $20\left(M \approx 25 \mathrm{M}_{\odot}\right)$, les températures peuvent ainsi augmenter de quelques $10^{6} \mathrm{~K}$ à environ $4 \times 10^{9} \mathrm{~K}$. Cette augmentation de température permet la réalisation de l'équilibre énergétique par des combustions nucléaires impliquant des noyaux de plus en plus chargés (l'augmentation de température est en effet associée à des énergies d'intérêt astrophysique croissantes, et donc à la possibilité de pénétration de barrières coulombiennes de plus en plus élevées; voir P. Descouvemont, ce volume). Cette augmentation de la température des phases de combustion est accompagnée d'une diminution drastique de la durée de ces phases. Cette situation résulte de la combinaison de (i) une diminution du taux de production d'énergie en passant de la combustion de $\mathrm{H}$ à des phases plus avancées de l'évolution et (ii) une production accrue des neutrinos, et par conséquent des pertes d'énergie, lorsque les températures excèdent $5 \times 10^{8} \mathrm{~K}$ environ ([11] Chap. 10). La Fig. 20 montre également de façon extrêmement schématique qu'une phase de combustion, une fois terminée au coeur de l'étoile, se prolonge dans une couche stellaire périphérique plus. ou moins mince. Ainsi, les régions stellaires profondes développent une structure en 'pelures d'oignon' de compositions différentes et allant en se complexifiant au fil du temps.

Il est important de noter que toutes les étoiles ne passent pas nécessairement par toutes les phases nucléaires apparaissant à la Fig. 20 ([11] Chap. 6.6 pour plus de détails). Alors que les étoiles massives $\left(M \gtrsim 10 \mathrm{M}_{\odot}\right.$ connaissent toutes les combustions indiquées, l'histoire nucléaire des étoiles de masse faible $\left(0.45 \lesssim M \lesssim 8 \mathrm{M}_{\odot}\right)$ s'arrête dès la fin de la combustion centrale de $\mathrm{He}$. Les étoiles de masse plus faible encore ne brûlent pas leur He ou même leur 


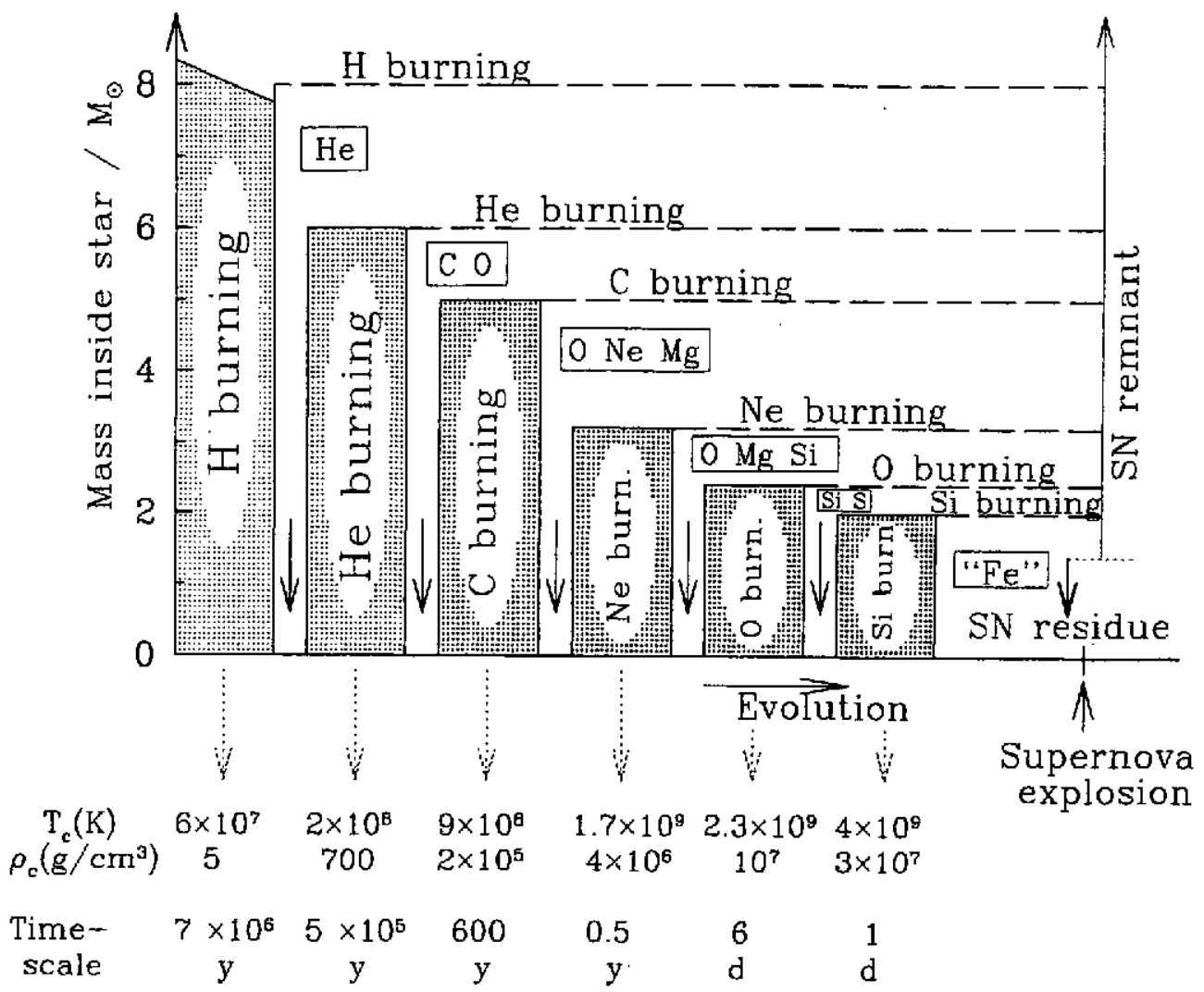

Figure 20. Représentation très schématique de l'évolution de la structure interne d'une étoile sphérique de masse $M \approx 25 \mathrm{M}_{\odot}$. En ordonnée est portée la fraction de masse contenue à l'intérieur d'une sphère de rayon donné, rapportée à la masse du Soleil (le centre de l'étoile est donc à l'origine des ordonnées). Les zones hachurées représentent des phases de combustion nucléaire capable d'assurer l'équilibre énergétique. Une phase de combustion donnée débute dans le coeur de l'étoile (les températures centrales $T_{\mathrm{c}}$ et densités centrales $\rho_{c}$ sont indiquées sous la figure) avant de se déplacer dans une zone périphérique mince. Les phases de combustion nucléaire sont entrecoupées de stades de contraction gravitationnelle (flèches descendantes) quasi-hydrostatiques, du moins jusqu'à la combustion du Si. Les symboles chimiques représentent les éléments les plus abondants présents à la fin d'une combustion nucléaire donnée ('Fe' symbolise les noyaux du pic du fer dont le nombre de masse $A$ est compris entre 50 et 60 environ). Si l'étoile massive considérée explose en supernova (de Type II), ses régions les plus internes peuvent laisser un 'résidu', tandis que le reste de la matière est éjecté dans le MIS (il sera appelé dans la suite 'éjecta' de la supernova)

$\mathrm{H}$ (si $M \lesssim 0.08 \mathrm{M}_{\odot}$ ) et ne seront pas discutées ici. Les étoiles dans le domaine $8 \lesssim M \lesssim 10$ $\mathrm{M}_{\odot}$, qui constituent des cas intermédiaires très complexes, ne seront pas considérées non plus. Il convient également de souligner que la véritable structure stellaire est certainement bien plus compliquée que celle schématisée à la Fig. 20 (voir à titre illustratif les Figs. 10.5 et 10.6 de [11]), même si toute déviation à la symétrie sphérique globale est négligée. Cette symétrie sphérique peut elle-même être violée dans certaines régions d'une étoile, en particulier durant certaines phases évolutives avancées. Dans ces conditions, la structure et l'évolution stellaires atteignent un haut niveau de complexité, mise en évidence par des simulations multi-dimensionnelles récentes $([109,110])$. La rotation introduit évidemment des difficultés supplémentaires ([111]). Enfin, des vents stellaires continus peuvent également affecter l'évolution de diverses façons $([81,112])$. 
6.4.1. Etoiles de masse $M \gtrsim 10 M_{\odot}$ L'évolution de ces étoiles et la nucléosynthèse concomitante ont fait l'objet de multiples travaux, du moins sous l'hypothèse de la symétrie sphérique ([11, 79, 113]). Après avoir subi toutes les phases de combustion nucléaire apparaissant à la Fig. 20, ces étoiles possèdent un coeur de fer dépourvu de sources d'énergie nucléaire, toute transformation des noyaux de Fe très liés étant endothermique. En fait, ce coeur hors d'équilibre énergétique voit son exposant polytropique $\Gamma$ devenir inférieur à $4 / 3$ sous l'effet de captures d'électrons libres et de la photodésintégration du fer. Il en résulte une instabilité dynamique du coeur stellaire et son implosion (voir Fig. 17). Une chaîne extrêmement complexe de phénomènes physiques, dont l'étude sort largement du cadre de ces leçons, peut transformer cette implosion en une spectaculaire explosion appelée supernova de Type II (SNII) $\ddagger$ (voir [11], Chaps. 12 et 13 en particulier, pour une discussion détaillée du mécanisme d'implosion-explosion). Dans cette séquence d'événements physiques, la production et la diffusion de neutrinos dans le coeur de l'étoile jouent un rôle essentiel. En particulier, ces processus neutriniques aident à la formation et à la propagation d'une onde de choc au travers des diverses couches de l'étoile schématisées à la Fig. 20. L'onde de choc comprime ces couches et les échauffe avant de provoquer leur éjection dans le MIS, et donc leur refroidissement. Ce processus d'échauffement et de refroidissement associé au passage de l'onde de choc permet à certaines transformations de se produire pendant un temps relativement bref, modifiant ainsi plus ou moins profondément la composition pré-explosive des différentes couches stellaires. L'étude de la composition résultante du matériau éjecté constituant l'éjecta de la supernova est l'un des principaux chapitres de la théorie de la 'nucléosynthèse explosive'.

Dans ce scénario de SNII, toute la matière stellaire n'est pas retournée au MIS. Les régions les plus profondes sont en effet piégées dans un 'résidu' qui peut être une étoile à neutrons (observable sous la forme d'un pulsar si elle est magnétisée et en rotation rapide) ou même un trou noir ([116, 117] pour des détails sur la physique de ces résidus).

Les SNII brièvement décrites ci-dessus ne sont pas le seul mécanisme par lequel les étoiles massives peuvent restituer de la matière au MIS. Des étoiles chaudes (en surface) possèdent en effet des vents stellaires continus (non explosifs) qui peuvent éjecter du matériau n'ayant subi aucune nucléosynthèse explosive. Il en est tout particulièrement ainsi des étoiles WolfRayet qui enrichissent le MIS en produits de la combustion 'froide' de $\mathrm{H}$ et de $\mathrm{He}$ (voir Sect. 8; [118]). Ces étoiles peuvent d'ailleurs terminer leur évolution sous forme d'une supernova de type SNIb/Ic et ainsi apporter leur contribution à la contamination du MIS en produits de combustion explosive [119].

En conclusion, les étoiles massives, aussi bien au travers de leurs phases évolutives non explosives qu'explosives, constituent des agents essentiels dans l'évolution de la composition des galaxies. Il est également admis par certains que quelques grains isotopiquement anormaux identifiés dans diverses météorites se sont formés autour de supernovae. Si un consensus général semble aujourd'hui atteint sur les aspects généraux des SNII, bien des questions de physique nucléaire ou d'astrophysique restent largement non résolues, même dans le cas de modèles à une dimension. Que dire alors de la modélisation multidimensionnelle indispensable au traitement raisonnablement réaliste de certains aspects essentiels d'une explosion supernova! ([120]). Un tel traitement apparaît d'autant plus

$\ddagger$ Les SNII sont définies comme les supernovae présentant des raies de $\boldsymbol{H}$ dans leur spectre. II est vraisemblable que la plupart des étoiles massives, sinon toutes, possèdent encore une enveloppe d'hydrogène au moment de leur explosion, et sont donc des SNII. Au contraire, les supernovae sont dites de Type I (SNI) si aucune raie de $\mathbf{H}$ n'est visible dans leur spectre. Des caractéristiques spectrales particulières conduisent à l'identification de diverses sous-classes de SNI, parmi lesquelles les objets de type SNIa, Ib et Ic (voir $[114,115]$ pour plus de détails au sujet de la classification des supernovae) 
nécessaire que les observations mettent de plus en plus clairement en évidence l'existence de nombreux éjecta de supernovae relativement jeunes présentant des écarts plus ou moins significatifs à la symétrie sphérique ([121]).

6.4.2. Etoiles de masse $0.45 \lesssim M \lesssim 8 M_{\odot}$ Comme noté précédemment, l'histoire nucléaire de ces étoiles se limite aux combustions de $\mathrm{H}$ et de $\mathrm{He}$ qui débutent dans les régions centrales avant de se développer dans des couches périphériques. Cette activité nucléaire est responsable des multiples particularités de composition observées à la surface d'une grande variété d'étoiles RGB ou AGB où les produits de combustion sont amenés par divers mécanismes de transport ('dredge-up') ([122]; voir aussi S. Goriely, ce volume). Le niveau de cette contamination de l'atmosphere stellaire est également dépendant des vents très intenses que subissent les étoiles considérées au cours de la phase AGB. Ces pertes de masse continues sont responsables de la formation de nébuleuses planétaires, dont les résidus centraux évoluent vers des naines blanches essentiellement composées de $C$ et $O$, ainsi que de l'enrichissement des galaxies en produits des combustions de $\mathrm{H}$ et de $\mathrm{He}$. Il faut également souligner que certains grains isotopiquement anormaux observés dans diverses météorites proviennent sans doute d'enveloppes circumstellaires formées par les vents des étoiles AGB.

La modélisation des étoiles dans le domaine de masses considéré souffre encore de nombreuses incertitudes. Celles-ci concernent en particulier l'efficacité des épisodes de dredge-up et donc le niveau de contamination des surfaces stellaires par les produits des combustions de $\mathrm{H}$ et de He. L'intensité des vents stellaires en fonction du temps reste également mal connue et contribue aux incertitudes sur les composition de surface et sur le rôle exact joué par ces étoiles dans l'évolution chimique des galaxies. Enfin, la quantité de neutrons que peut produire une étoile AGB dans sa couche périphérique de combustion de $\mathrm{He}$, et donc les caractéristiques du processus s dans ces étoiles, reste l'un des problèmes non résolus majeurs qui touche de très près l'astrophysique nucléaire (voir S. Goriely, ce volume).

\subsubsection{Quelques brèves considérations au sujet des étoiles binaires Approximativement 2/3} de toutes les étoiles de notre Galaxie appartiennent à des systèmes multiples (essentiellement binaires) gravitationnellement liés. Différents types d'événements astronomiques observés sont aujourd'hui interprétés en termes de phénomènes spécifiques susceptibles de se produire dans de tels systèmes. Les effets d'un compagnon sur l'évolution d'une étoile dans un système binaire sont complexes et souvent loin d'être bien compris. Ceci concerne à la fois des problèmes purement structurels aussi bien que des questions de physique nucléaire particulières (voir [123] pour quelques revues). Nous nous limitons ici à quelques brefs commentaires ayant un rapport direct avec l'astrophysique nucléaire.

L'une des caractéristiques les plus importantes de l'évolution de systèmes binaires est l'existence d'épisodes d'échange de matière entre les étoiles de ces systèmes offrant une grande variété de scénarios astrophysiques et nucléaires spécifiques. Ainsi, diverses instabilités liées à la production d'énergie nucléaire dans la matière transférée peuvent prendre naissance à la surface de l'étoile subissant l'accrétion. En particulier, l'hydrogène peut brûler de façon explosive à la surface d'une naine blanche ou d'une étoile à neutrons et conduire respectivement au phénomène 'nova' ou 'sursaut $x$ '. En plus de ces phénomènes de surface, une naine blanche sur laquelle s'accumule de la matière en provenance d'un compagnon peut également subir en son intérieur des combustions explosives de $\mathrm{He}$ ou $\mathrm{C}$ de type détonation ou déflagration (Sect. 10.1), différentes combustions et différents régimes de combustion pouvant dans certains cas se développer de façon séquentielle dans le même objet. Il peut en résulter l'explosion de la naine blanche en supernova de Type Ia ([124]). Ces explosions constituent 


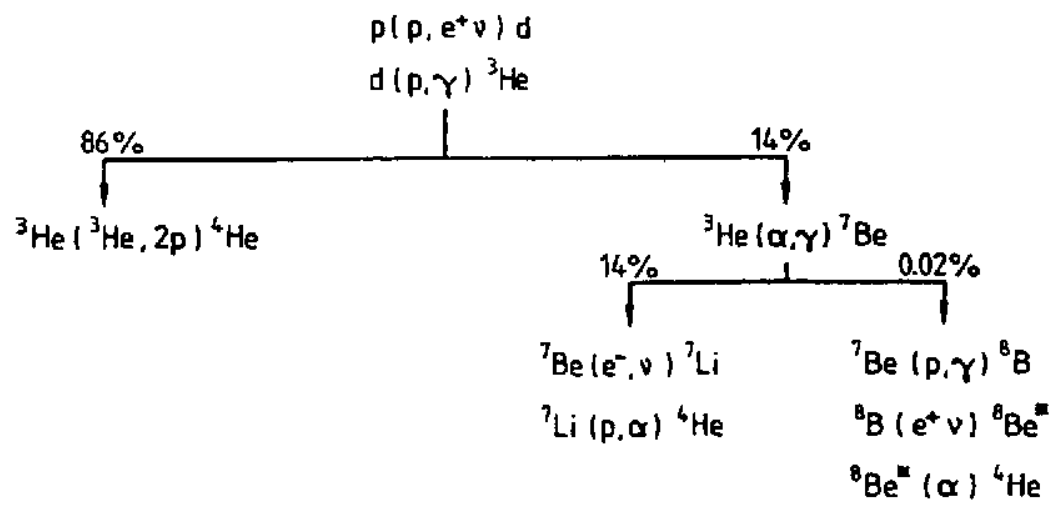

CHAIN I

$\mathrm{Q}_{\mathrm{eff}}=26.20 \mathrm{MeV}$

$(2.0 \%$ loss)
CHAIN II

$Q_{\text {eff }}=25.66 \mathrm{MeV}$

$(4.0 \%$ loss)

\section{CHAIN III}

$$
Q_{\text {eff }}=19.17 \mathrm{MeV}
$$

(28.3\% loss)

NET - RESULT : $4 p-{ }^{4} \mathrm{He}+2 \mathrm{e}^{+}+2 \mathrm{v}+\mathrm{a}_{\mathrm{eff}}$

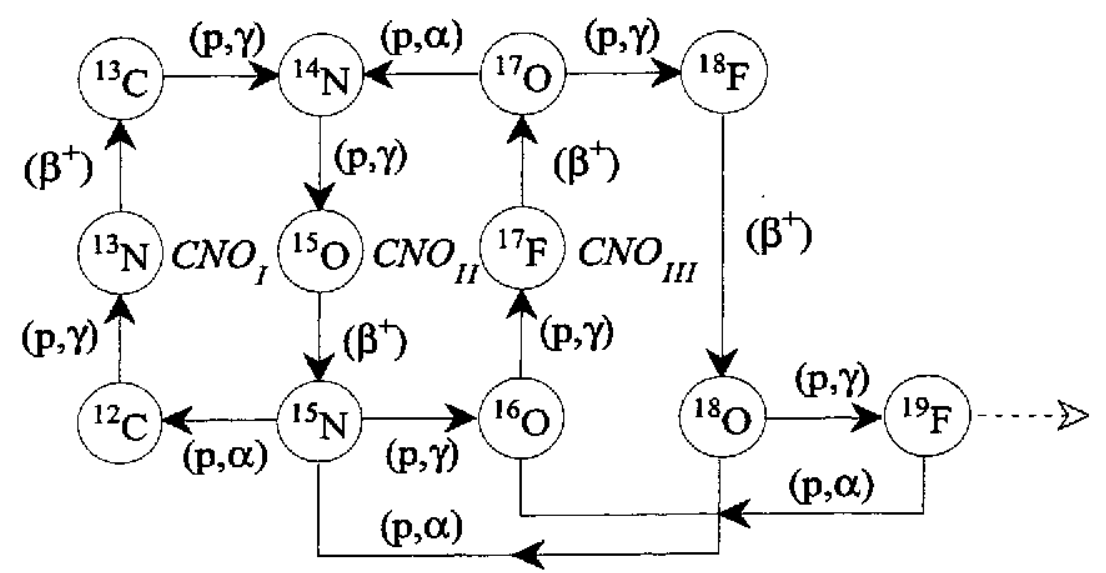

Figure 21. (a) Les chaînes proton-proton froides. Elles se développent à des températures $T \gtrsim 10^{6} \mathrm{~K}$ et ont pour effet principal de transformer $\mathrm{H}$ en ${ }^{4} \mathrm{He}$. Les valeurs de $Q_{\text {eff }}$ prennent en compte la perte d'énergie par les neutrinos produits. Les rapports de branchement indiqués se rapportent aux conditions régnant au centre du Soleil; (b) Les cycles CNO froids. Ils se développent à $T \gtrsim 10^{7} \mathrm{~K}$. Ils transforment également $\mathrm{H}$ en ${ }^{4} \mathrm{He}$, ainsi que la presque totalité des noyaux de $\mathrm{C}, \mathrm{N}$ et $\mathrm{O}$ en ${ }^{14} \mathrm{~N}$

des agents importants de l'évolution chimique des galaxies. Sous certaines circonstances, la naine blanche pourrait au contraire imploser et former une étoile à neutrons.

\section{Quelques généralités sur la nucléosynthèse stellaire non explosive}

Nous decrivons dans ce chapitre quelques chaînes de réactions qui jouent un rôle important dans l'établissement de l'équilibre énergétique et/ou dans la nucléosynthèse lors des phases non explosives de l'évolution stellaire schématisées à la Fig. 20. 


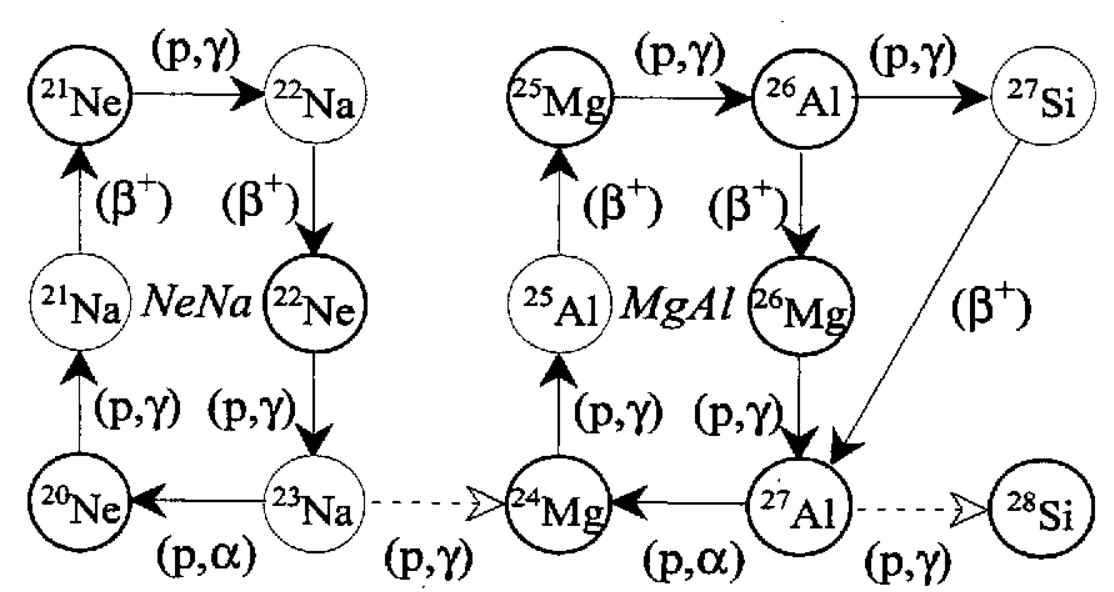

Figure 22. Réactions impliquées dans les chaînes $\mathrm{NeNa}$ et $\mathrm{MgAl}$ froides. Elles se développent principalement à $T \gtrsim(2-3) \times 10^{7} \mathrm{~K}$

\subsection{Les modes 'froids' de combustion de l'hydrogène}

La combustion non explosive de l'hydrogène peut s'opérer selon différents modes ([10]), appelés 'froids' dans la suite dans la mesure où ils se développent à des températures plus basses que celles rencontrées dans des situations explosives (voir Sect. 9). L'importance relative de ces modes dépend des conditions de température et de densité, et donc de la masse initiale des étoiles, ainsi que de leur composition initiale. Les chaînes proton-proton et les cycles CNO correspondent globalement à la transformation de $\mathrm{H}$ en ${ }^{4} \mathrm{He}$ (Fig. 21) et constituent une source essentielle d'énergie nucléaire respectivement dans les étoiles de masse $M \lesssim 1.5 \mathrm{M}_{\odot}$ et dans les étoiles plus massives, ces domaines de masse correspondant à une composition initiale proche de celle du Soleil. Cette importante production d'énergie est responsable de la séquence principale du DHR (Figs. 1 et 2). Les chaînes proton-proton sont également les producteurs essentiels des neutrinos émis par le Soleil; à ce titre, de très nombreuses études de physique nucléaire expérimentale et théorique leur ont été consacrées et continuent à l'être ([78]). Cet effort concerne en particulier ${ }^{3} \mathrm{He}\left({ }^{3} \mathrm{He}, 2 \mathrm{p}\right){ }^{4} \mathrm{He}$, aujourd'hui la seule réaction dont la section efficace a pu être mesurée dans le domaine d'énergie d'intérêt solaire, et la réaction ${ }^{7} \mathrm{Be}(\mathrm{p}, \gamma){ }^{8} \mathrm{~B}$ responsable de la production des neutrinos qui sont à l'origine du problème des neutrinos solaires. D'autre part, le cycle $\mathrm{NeNa}$ froid et la chaîne $\mathrm{MgAl}$ froide sont deux autres modes de combustion de $\mathrm{H}$ (Fig. 22) qui, s'ils ne jouent aucun rôle énergétique significatif, sont importantes sur le plan nucléosynthétique [à noter en particulier que le radionucléide ${ }^{26} \mathrm{Al}$ de grande importance astrophysique (Sect. 1) est impliqué dans la chaîne $\mathrm{MgAl}$. Quelques détails relatifs à la nucléosynthèse accompagnant les cycles $\mathrm{CNO}$ et $\mathrm{NeNa}$, ainsi que la chaîne $\mathrm{MgAl}$ sont fournis aux Figs. 23 et 24, qui mettent notamment en évidence les incertitudes d'origine purement nucléaire affectant les prédictions d'abondances. Les incertitudes sur quelques vitesses sont illustrées à la Fig. 25 (voir [78] pour plus de détails)

\subsection{La combustion de l'hélium et la production de neutrons}

La combustion non explosive de He implique un ensemble de réactions dont les principales apparaissent à la Fig. 26. Lorsqu'elle se produit dans les régions centrales de l'étoile (voir Fig. 20), elle assure l'équilibre énergétique dès que la température excède $10^{8} \mathrm{~K}$ environ. Cette fusion nucléaire est également d'une grande importance sur le plan nucléosynthétique. 

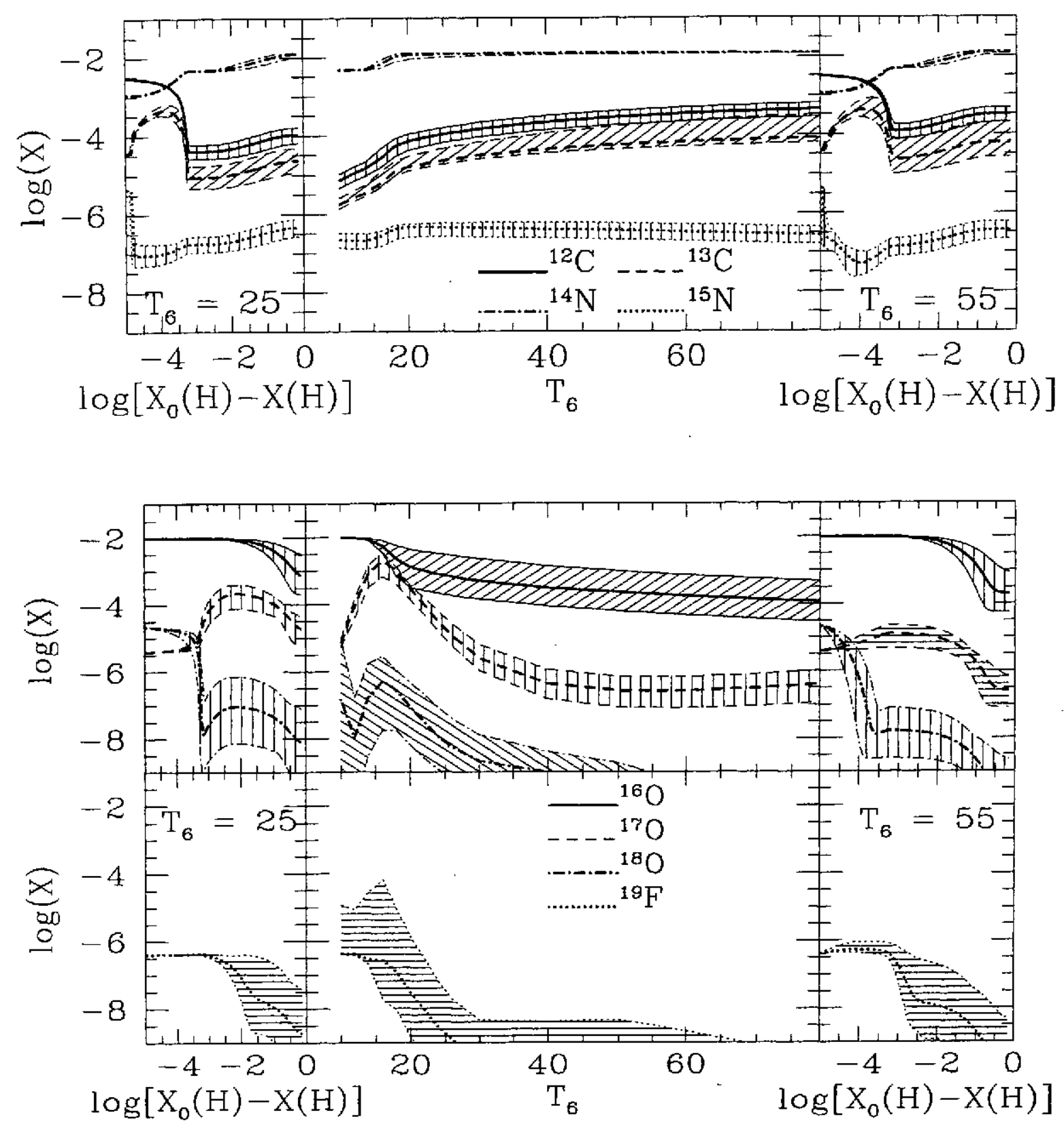

Figure 23. Gauche et droite: variations temporelles des fractions massiques des isotopes stables de $\mathrm{C}, \mathrm{N}, \mathrm{O}$ et $\mathrm{F}$ en fonction de la quantité de $\mathrm{H}$ brûlé dans le cadre d'un modèle paramétrique simple supposant une densité constante $\left(\rho=100 \mathrm{gcm}^{-3}\right)$ et des températures constantes $\left(T_{6}=25\right.$ et 55 , où $T_{6}$ est la température exprimée en $\left.10^{6} \mathrm{~K}\right)$. Les abondances initiales sont supposées solaires [30]. $\mathrm{X}(\mathrm{H})$ est la fraction massique de $\mathrm{H}$, l'indice 0 correspondant à sa valeur initiale. Milieu: fractions massiques des mêmes noyaux à la fin de la combustion de $\mathrm{H}\left[X(\mathrm{H})=10^{-5}\right]$ en fonction de $T_{6}$. Les zones hachurées délimitent les incertitudes résultant des taux de réactions [78]. Outre la production de ${ }^{4} \mathrm{He}$ à partir de $\mathrm{H}$, les cycles CNO transforment la plus grande partie de $\mathrm{C}, \mathrm{N}$ et $\mathrm{O}$ en ${ }^{14} \mathrm{~N}$ (figure tirée de [94]) 

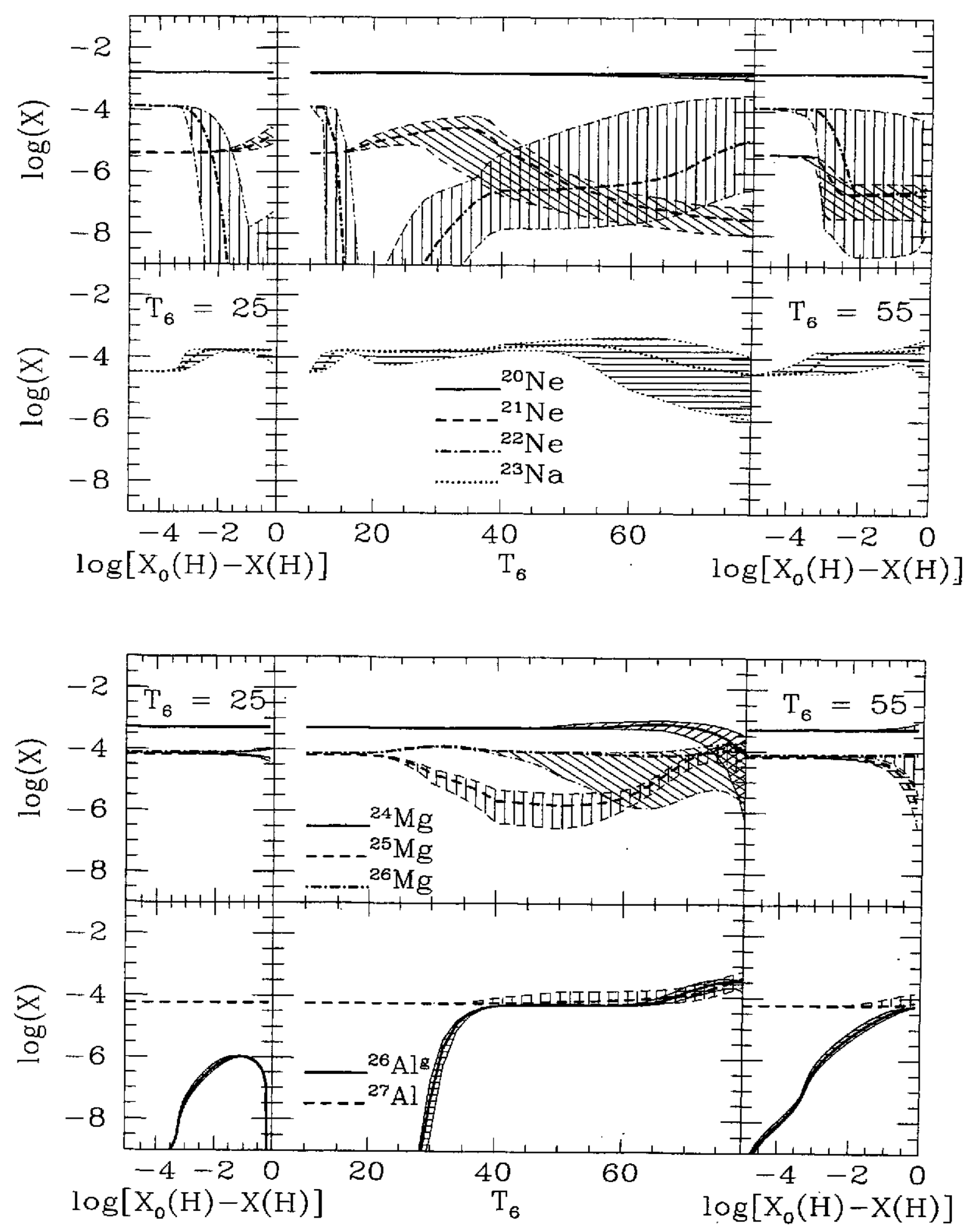

Figure 24. Identique à la Fig. 23, mais pour les noyaux impliqués dans les chaînes $\mathrm{NeNa}$ et $\mathrm{MgAl}$. Le caractère cyclique de la chaîne NeNa n'est assuré que si ${ }^{23} \mathrm{Na}(\mathrm{p}, \alpha){ }^{20} \mathrm{Ne}$ est plus rapide que ${ }^{23} \mathrm{Na}(p, \gamma){ }^{24} \mathrm{Mg}$. Les vitesses adoptées fournies par NACRE [78] confirment une telle possibilité, du moins pour $T \leqq 5 \times 10^{7} \mathrm{~K}$. Cependant, les incertitudes subsistant dans la vitesse de transformation de ${ }^{23} \mathrm{Na}$ en ${ }^{24} \mathrm{Mg}$ ne permettent pas de formuler une conclusion définitive à ce sujet. A noter tout particulièrement la production de ${ }^{26} \mathrm{Al}$ par la chaîne $\mathrm{MgAl}$ 

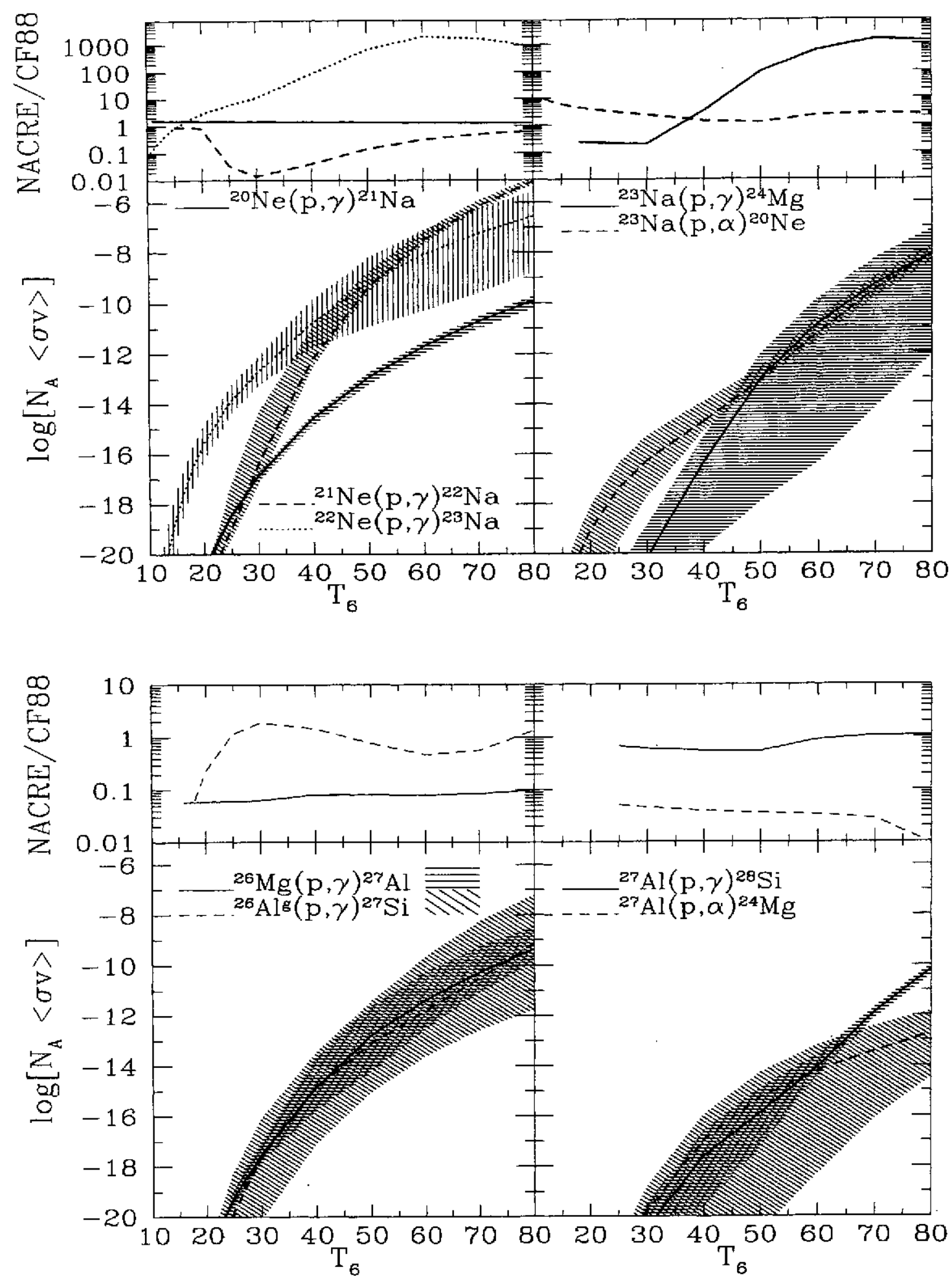

Figure 25. Dépendance en la température de quelques vitesses stellaires de captures de protons [exprimées en $\mathrm{cm}^{3} \mathrm{~mol}^{-1} \mathrm{~s}^{-1}$ ] impliquées dans les chaînes $\mathrm{NeNa}$ (haut) et $\mathrm{MgAl}$ (bas). Les incertitudes sur les vitesses 'adoptées' fournies par la compilation NACRE [78] sont représentées par les zones hachurées. Les rapports entre les vitesses 'adoptées' par NACRE et par une compilation précédente (CF88; [93]) largement utilisée dans les calculs astrophysiques sont également fournis 


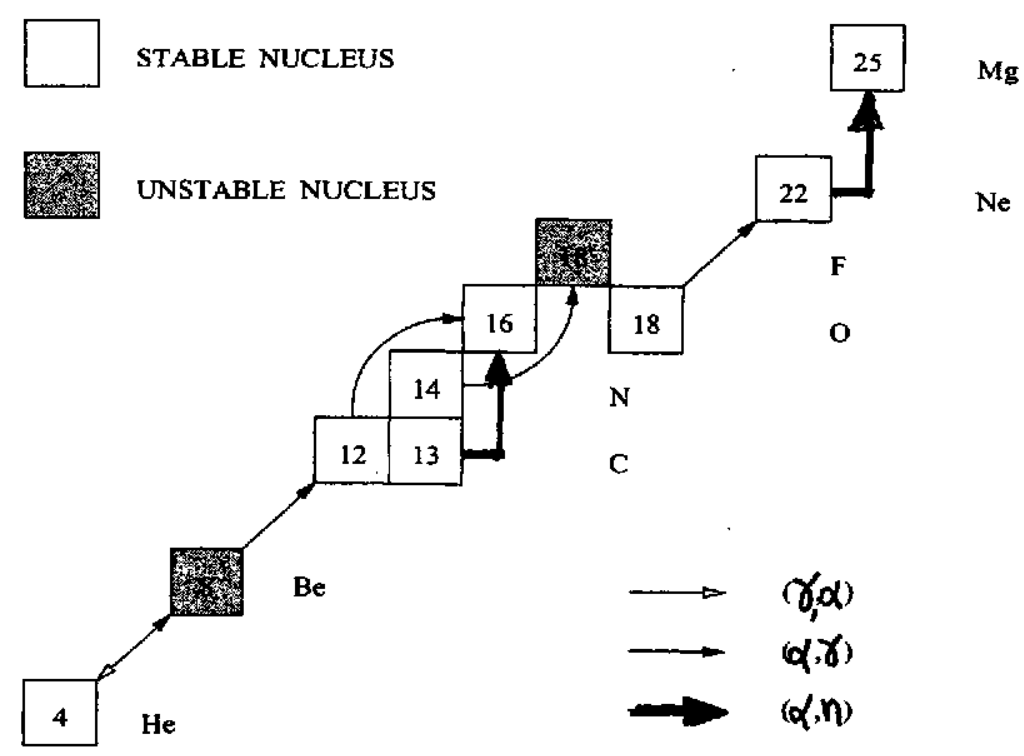

Figure 26. Réactions principales impliquées dans la combustion non explosive de He

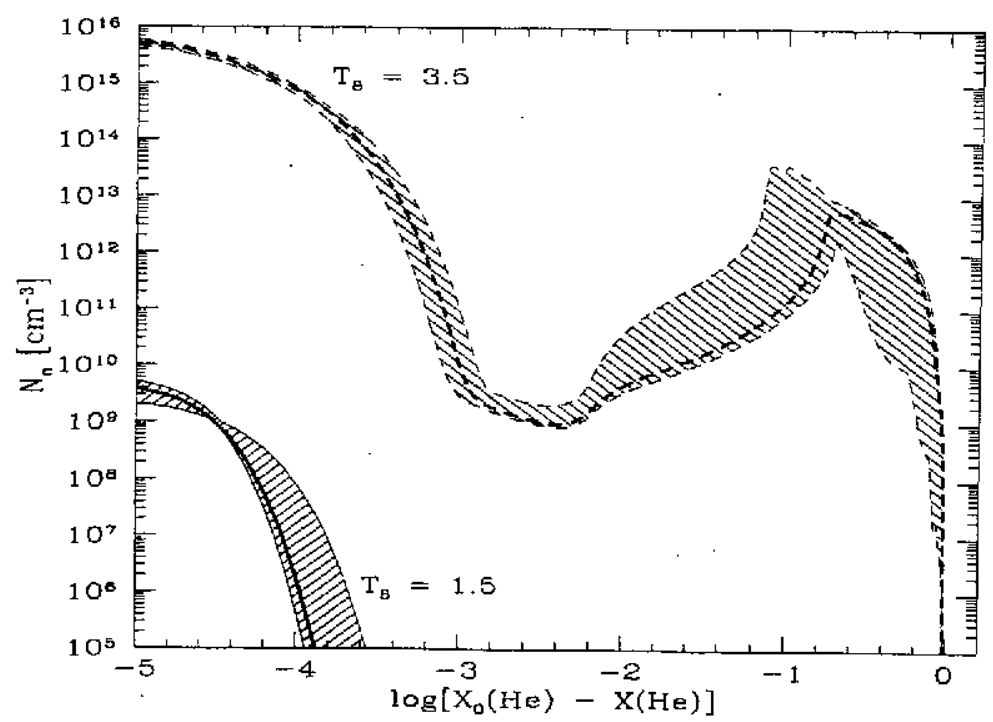

Figure 27. Densité de neutrons $N_{n}$ en fonction de là quantité de He brûlé $\left[\mathrm{X}_{0}(\mathrm{He})\right.$ étant la fraction massique initiale de He] à $\rho=10^{4} \mathrm{gcm}^{-3}$ et $T_{8}=1.5$ ou $T_{8}=3.5\left(T_{8}\right.$ est la température exprimée en $10^{8} \mathrm{~K}$ ). Les traits pleins et pointillés sont obtenus sur base des valeurs 'adoptées' de la compilation NACRE [78], tandis que les zones grisées fournissent les domaines de variation de $N_{n}$ associés aux incertitudes nucléaires. La fraction massique initiale de ${ }^{13} \mathrm{C}$ est prise égale à $10^{-4}$, valeur obtenue à la fin des cycles CNO opérant à $T_{6}=60$ et $\rho=100 \mathrm{gcm}^{-3}$

Elle est en effet responsable de la production de ${ }^{12} \mathrm{C}$ et d'une partie au moins ${ }^{2}{ }^{26} \mathrm{O}$, ces deux nucléides jouant un rôle clé non seulement dans l'évolution ultérieure de l'étoile, mais également dans l'évolution chimique et biologique de la Galaxie. D'autre part, la combustion de He fournit la première source possible de neutrons au travers des réactions ${ }^{13} \mathrm{C}(\alpha, \mathrm{n}){ }^{16} \mathrm{O}$ ou ${ }^{22} \mathrm{Ne}(\alpha, \mathrm{n}){ }^{25} \mathrm{Mg}$. L'importance relative de ces deux réactions dans le développement du processus s de captures de neutrons (voir S. Goriely, ce volume) dépend fortement des conditions astrophysiques, et en particulier de la masse des étoiles et de leur phase évolutive. 
Basic:

$$
\begin{aligned}
& { }^{2} \mathrm{C}\left({ }^{12} \mathrm{C}, \begin{array}{l}
\alpha \\
p
\end{array}\right){ }^{23} \mathrm{Ne} \\
& { }^{23} \mathrm{Na}
\end{aligned}
$$

Figure 28. Réactions importantes dans la combustion non explosive du C [80]. Les cendres principales de cette combustion sont les noyaux $20 \lesssim A \lesssim 27$. Des neutrons peuvent être libérés et conduire à un processus s limité

En raison de son importance, la fusion de He a fait l'objet d'un très grand nombre de travaux tant sur le plan astrophysique que sur celui de la physique nucléaire expérimentale et théorique. La réaction ${ }^{12} \mathrm{C}(\alpha, \gamma){ }^{16} \mathrm{O}$ a fait l'objet d'une attention toute particulière $([10,78])$ en raison de son impact sur l'évolution de l'étoile après la phase de combustion centrale de He et sur la nucléosynthèse concomitante ([79]). De grandes incertitudes affectent également bien d'autres réactions impliquées dans la combustion de $\mathrm{He}$ [78]. L'impact de ces incertitudes sur la nucléosynthèse associée a été évalué par [94] sur base d'un modèle astrophysique simple. Cette étude concerne en particulier les réactions de type $(\alpha, \mathbf{n})$ produisant des neutrons (notamment à partir de ${ }^{13} \mathrm{C}$ ou ${ }^{22} \mathrm{Ne}$ ), et donc l'efficacité du processus $\mathrm{s}$ au travers de la densité de neutrons produits (voir Fig. 27). Plus spécifiquement, [125] analyse le rôle des grandes incertitudes subsistant dans la vitesse de ${ }^{22} \mathrm{Ne}(\alpha, \mathrm{n}){ }^{25} \mathrm{Mg}$ sur le processus s dans des étoiles massives et sur le processus p subséquent (voir aussi S. Goriely, ce volume).

\subsection{Les combustions de $\mathrm{C}, \mathrm{Ne}, \mathrm{O}$, Si et l'équilibre nucléaire statistique}

La Fig. 28 fournit les réactions les plus importantes accompagnant la combustion du $\mathrm{C}$, qui peut assurer l'équilibre énergétique de l'étoile lorsque les températures centrales atteignent approximativement $6 \times 10^{8} \mathrm{~K}$. L'importance de cette phase nucléaire dans le bilan énergétique et la nucléosynthèse dépend essentiellement de la quantité de ${ }^{12} \mathrm{C}$ produite à la fin de la combustion de He, et est donc fonction du taux de la réaction ${ }^{12} \mathrm{C}(\alpha, \gamma){ }^{16} \mathrm{O}$. Les neutrons pouvant être produits pendant cette combustion sont susceptibles de conduire au développement d'un processus s limité.

Cette phase de combustion soulève l'intéressante question de la fusion des ions lourds légers sous la barrière coulombienne, et en particulier de l'origine des structures très prononcées observées dans la section efficace de la réaction ${ }^{12} \mathrm{C}+{ }^{12} \mathrm{C}$ à ces basses énergies. Les études expérimentales et théoriques ne fournissent pas d'explication entièrement 
satisfaisante à ces structures $[10,84,85]$.

La combustion non-explosive de Ne est en réalité une phase nucléaire dominée pour la première fois dans l'évolution d'une étoile par une réaction de photodésintégration, en l'occurence ${ }^{20} \mathrm{Ne}(\gamma, \alpha){ }^{16} \mathrm{O}$ (voir Fig. 29). Outre ${ }^{16} \mathrm{O}$, les isotopes de $\mathrm{Mg}$, Al et $\mathrm{P}$ sont les nucléides les plus abondamment synthétisés lors de cette phase. Quelques neutrons peuvent également être produits et être responsables d'un processus s restreint.

La combustion non explosive de $\mathrm{Ne}$ précède celle de $\mathrm{O}$ qui peut assurer l'équilibre énergétique de l'étoile lorsque les températures centrales atteignent $2 \times 10^{9} \mathrm{~K}$ environ. $\S$. La Fig. 29 fournit les réactions les plus importantes de la combustion de $O$, qui produit essentiellement des nucléides dans le domaine $28 \lesssim A \lesssim 46$. Il est intéressant de noter le rôle croissant des captures d'électrons libres, qui s'accentue lors des phases évolutives ultérieures. Il faut également souligner que la fusion ${ }^{16} \mathrm{O}+{ }^{16} \mathrm{O}$ ne présente pas les problèmes nucléaires posés par ${ }^{12} \mathrm{C}+{ }^{12} \mathrm{C}$. Il n'en reste pas moins que beaucoup doit encore être fait en vue de comprendre la physique des ions lourds à des énergies sub-coulombiennes.

Enfin, la combustion du $\mathrm{Si}$, qui met en jeu un ensemble très complexe de réactions opérant à des températures supérieures à $4 \times 10^{8} \mathrm{~K}$ environ ([10]), doit plutôt être comprise comme une 'fonte' du Si. Cette transformation est faite d'un flot nucléaire partant de ${ }^{28} \mathrm{Si}$ et évoluant vers des nucléides de plus en plus légers. Ce flot est associé aux réactions

$$
\begin{aligned}
& \gamma+{ }^{28} \mathrm{Si} \leftrightarrow \leftrightarrow^{27} \mathrm{Al}+p ; \gamma+{ }^{28} \mathrm{Si} \leftrightarrow \leftrightarrow^{24} \mathrm{Mg}+\alpha \\
& \gamma+{ }^{24} \mathrm{Mg} \leftrightarrow{ }^{23} \mathrm{Na}+p ; \gamma+{ }^{24} \mathrm{Mg} \leftrightarrow{ }^{20} \mathrm{Ne}+\alpha \\
& \gamma+{ }^{20} \mathrm{Ne} \leftrightarrow{ }^{16} \mathrm{O}+\alpha \\
& \gamma+{ }^{16} \mathrm{O} \leftrightarrow{ }^{12} \mathrm{C}+\alpha,
\end{aligned}
$$

les photodésintégrations étant partiellement contrebalancées par les réactions inverses, comme symbolisé par les flèches doubles. Les particules $\alpha$ ou nuciéons ainsi libérés peuvent être capturés par le ${ }^{28} \mathrm{Si}$ restant, ce qui conduit au développement d'un flot nucléaire évoluant vers des nucléides de plus en plus lourds, ainsi que symbolisé par

$$
{ }^{28} \mathrm{Si}+\alpha \leftrightarrow{ }^{32} \mathrm{~S}+\gamma ;{ }^{32} \mathrm{~S}+\alpha \leftrightarrow{ }^{36} \mathrm{Ar}+\gamma ; \ldots \text { noyaux du groupe du fer. }
$$

Ce flot ascendant peut être symbolisé par

$$
\begin{aligned}
& 2\left({ }^{28} \mathrm{Si}\right) \rightarrow^{28} \mathrm{Si}+7 \alpha \rightarrow^{56} \mathrm{Ni} \\
& \text { ou } \\
& 2\left({ }^{28} \mathrm{Si}\right) \rightarrow{ }^{28} \mathrm{Si}+6 \alpha+2 n+2 p \rightarrow{ }^{54} \mathrm{Fe}+2 p,
\end{aligned}
$$

la transformation dominante dépendant des températures et densités auxquelles se déroule la combustion de Si.

L'ensemble des photodésintégrations et captures de particules dont question plus haut finit par conduire à la formation d'une situation d'équilibre nucléaire statistique entre des ensembles limités de noyaux généralement appelés des 'groupes en équilibre quasi-statistique' (EQS), connectés entre eux par des réactions trop lentes pour permettre la réalisation d'un équilibre plus large ([86]). Une telle situation est illustrée à la Fig. 30. Dans chaque groupe en EQS, les abondances relatives des différents membres sont indépendantes du temps et

$\S$ Les combustions de $\mathrm{Ne}$ et $\mathrm{O}$ peuvent être pratiquement concomitantes pour certaines masses stellaires 


\section{NEON BURNING}

Basic reactions:

${ }^{20} \mathrm{Ne}(\gamma, \alpha){ }^{16} \mathrm{O} \quad{ }^{20} \mathrm{Ne}(\alpha, \gamma){ }^{24} \mathrm{Mg}(\alpha, \gamma){ }^{28} \mathrm{Si}$

Flows $>10^{-2}$ times the above:

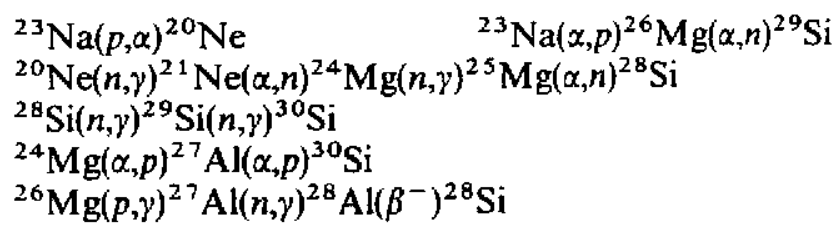

At low temperature and high density

$\left({ }^{22}\right.$ Ne left from prior n-rich $C$ burning):

${ }^{22} \mathrm{Ne}(\alpha, n){ }^{25} \mathrm{Mg}(n, \gamma){ }^{26} \mathrm{Mg}(n, \gamma){ }^{27} \mathrm{Mg}\left(\beta^{-}\right)^{27} \mathrm{Al}$

\section{OXYGEN BURNING}

Basic reactions:

$$
\begin{aligned}
& { }^{16} \mathrm{O}\left\{\begin{array}{l}
\left.\left({ }^{16} \mathrm{O}, p\right)\right)^{31} \mathrm{P} \\
\left({ }^{16} \mathrm{O}, \alpha\right)^{28} \mathrm{Si} \\
\left({ }^{16} \mathrm{O}, n\right)^{31} \mathrm{~S}\left(\beta^{+}\right)^{31} \mathrm{P}
\end{array}\right. \\
& { }^{31} \mathrm{P}(p, \alpha)^{28} \mathrm{Si}(\alpha, \gamma)^{32} \mathrm{~S} \\
& { }^{28} \mathrm{Si}(\gamma, \alpha)^{24} \mathrm{Mg}(\alpha, p)^{27} \mathrm{Al}(\alpha, p)^{30} \mathrm{Si} \\
& \left.{ }^{32} \mathrm{~S}(n, \gamma)^{33} \mathrm{~S}(n, \alpha)^{30} \mathrm{Si}(\alpha, \gamma)\right)^{34} \mathrm{~S} \\
& { }^{28} \mathrm{Si}(n, \gamma)^{29} \mathrm{Si}\left\{\begin{array}{l}
(\alpha, n){ }^{32} \mathrm{~S}(\alpha, p){ }^{35} \mathrm{Cl} \\
(p, \gamma)^{30} \mathrm{P}\left(\beta^{+}\right)^{30} \mathrm{Si}
\end{array}\right.
\end{aligned}
$$

Electron captures:

$$
\begin{aligned}
& \left.{ }^{33} \mathrm{~S}\left(e^{-}, v\right)\right)^{33} \mathrm{P}(p, n)^{33} \mathrm{~S} \\
& { }^{35} \mathrm{Cl}\left(e^{-}, v\right)^{35} \mathrm{~S}(p, n)^{35} \mathrm{Cl}
\end{aligned}
$$

Massive stars $\left(M_{\alpha}=16 M_{\odot}\right)$ :

$$
\begin{aligned}
& \left.{ }^{32} \mathrm{~S}(\alpha, \gamma)\right)^{36} \mathrm{Ar}\left\{\begin{array}{l}
(\alpha, p){ }^{39} \mathrm{~K} \\
(n, \gamma)^{37} \mathrm{Ar}\left(\beta^{+}\right)^{37} \mathrm{Cl}
\end{array}\right. \\
& { }^{35} \mathrm{Cl}\left\{\begin{array} { l } 
{ ( \gamma , p ) ^ { 3 4 } \mathrm { S } ( \alpha , \gamma ) ^ { 3 8 } \mathrm { Ar } } \\
{ ( e ^ { - } , \nu ) ^ { 3 5 } \mathrm { S } ( \gamma , p ) ^ { 3 4 } \mathrm { S } }
\end{array} \left\{\begin{array}{l}
(p, \gamma)^{39} \mathrm{~K}(p, \gamma){ }^{40} \mathrm{Ca} \\
(\alpha, \gamma)^{42} \mathrm{Ca}{ }_{(\alpha, p)^{45} \mathrm{Sc}(p, \gamma)^{46} \mathrm{Ti}}(\alpha, \gamma)
\end{array}\right.\right.
\end{aligned}
$$

Lower mass stars $\left(M_{\alpha}=4 M_{\odot}\right)$ :

$$
\begin{aligned}
& \left.{ }^{31} \mathrm{P}\left(e^{-}, v\right)\right)^{31} \mathrm{~S} \\
& { }^{32} \mathrm{~S}\left(e^{-}, v\right)^{32} \mathrm{P}(p, n)^{32} \mathrm{~S} \\
& { }^{31} \mathrm{P}(p, \alpha)^{30} \mathrm{Si}(n, \gamma){ }^{32} \mathrm{P}
\end{aligned}
$$

Figure 29. Réactions importantes dans la combustion non explosive de $\mathrm{Ne}$ (haut) et de $\mathrm{O}$ (bas) [80]. Le vocable Massive stars se rapporte à des étoiles dont la masse excède 20 à 25 $\mathrm{M}_{\odot}$ environ 


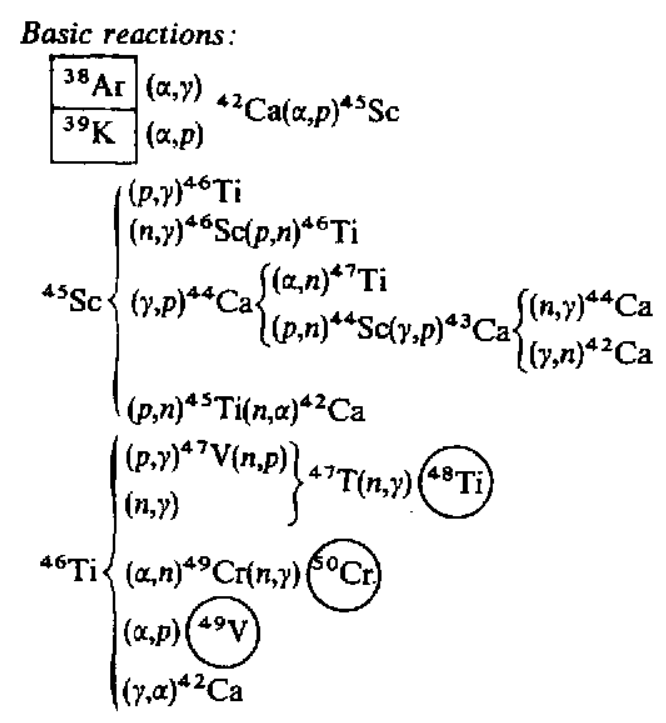

Additional reactions in high-temperature burning:

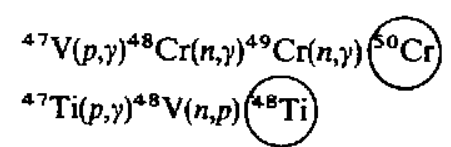

Important reactions in low-temperature burning:

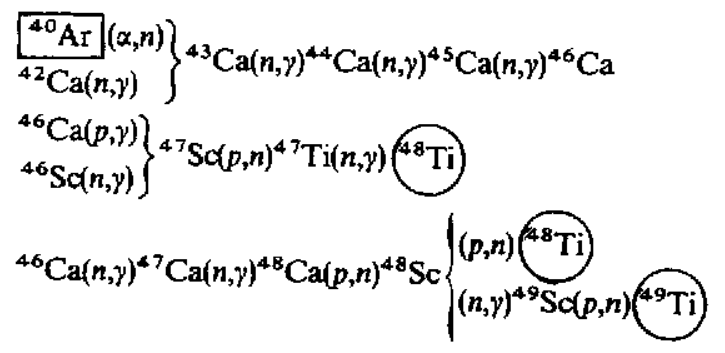

Figure 30. Réactions importantes reliant deux groupes en EQS lors de la combustion du $\mathrm{Si}[80]$. Les nucléides entourés d'un rectangle ou d'un cercle font partie du premier $(28 \leq A \leq 45)$ ou du second $(45 \leq A \leq 56)$ groupe en EQS

sont calculées à partir des équations de la mécanique statistique d'équilibre, qui ne font intervenir que la température, les énergies de liaison et les fonctions de partition nucléaire. $\mathrm{Au}$ contraire, les abondances relatives entre les différents groupes en EQS varient dans le temps, et leur évaluation repose sur la résolution d'un réseau de réactions, l'un de ces réseaux étant illustré à la Fig. 30.

Le nombre et l'extension des groupes en EQS varient évidemment dans le temps, de même que les réseaux de réactions les connectant. Vers la fin de la combustion du Si (fraction massique inférieure à environ 0.005), les deux EQS représentés à la Fig. 30 fusionnent, réalisant un équilibre statistique nucléaire complet entre tous les nucléides du Si au Fe. A ce stade, l'application de la mécanique statistique aux conditions astrophysiques atteintes conduit à la conclusion que l'étoile possède un coeur fait des nucléides du groupe du fer. 


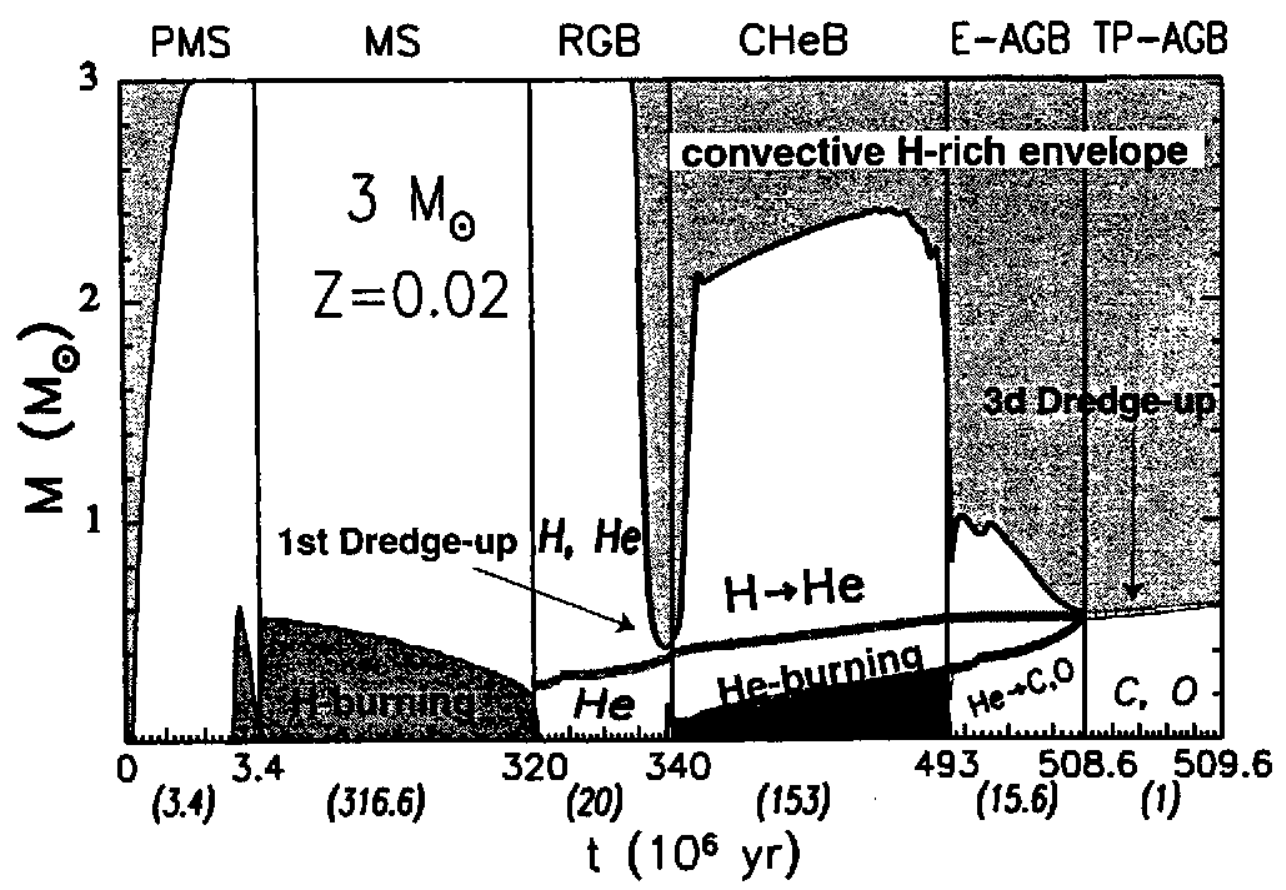

Figure 31. Evolution de la structure interne d'une étoile de $3 \mathrm{M}_{\odot}$ et de métallicité solaire $(Z=0.02)$ depuis la phase précédant la séquence principale (PMS) jusqu'à la phase AGB durant laquelle se développent des pulses thermiques récurrents (non représentés) entre les couches minces de combustion de $\mathrm{He}$ et de $\mathrm{H}$. Entre ces deux stades, l'étoile passe par la phase Séquence Principale (MS; combustion centrale de $\mathrm{H}$ ) et la phase Géante Rouge (RG; combustion centrale de $\mathrm{He} / \mathrm{combustion}$ périphérique de $\mathrm{H}$ ). Les zones en grisé s'étendant jusqu'à la surface correspondent à des régions où opère le tranport convectif de matière. Le 'premier dredge-up' contamine la surface en produits de la combustion centrale de $H$. Le second dredge-up, s'il opère $\left(M \gtrsim M_{\odot}\right)$ se développe à la fin de la combustion centrale de $\mathrm{He}$ et transporte en surface les produits de la combustion périphérique de $\mathrm{H}$. Enfin, le troisème drede-up se produit entre les pulses thermiques de la phase AGB et enrichit la surface en produits des combustions périphériques de $\mathrm{H}$ et de $\mathrm{He}$ (voir aussi S. Goriely, ce volume)

\section{La nucléosynthèse non explosive et l'observation astronomique}

La surface d'une grande variété d'étoiles peut être polluée à l'une ou l'autre phase de leur évolution par les produits de diverses combustions nucléaires se produisant dans des couches profondes. Cette contamination de surface fournit un moyen privilégié de confrontation entre l'observation astronomique et la modélisation des étoiles et de leur nucléosynthèse. La théorie prédit tout particulièrement la présence des produits de la combustion de l'hydrogène et de l'hélium à la surface de certains types d'étoiles. A titre d'exemple, il convient de citer les étoiles de masse faible ou intermédiaire $\left(M \lesssim 8 \mathrm{M}_{\odot}\right)$ lors des épisodes de 'dredgeup' (transport convectif de matière s'étendant depuis les zones de production de nucléides jusqu'en surface) pouvant se développer lors des phases Géante Rouge ou Géante Rouge Asymptotique (voir Fig. 31). Un autre exemple remarquable est fourni par les étoiles de type Wolf-Rayet (WR), étoiles très massives|| caractérisées par d'importantes pertes de masse pouvant atteindre des valeurs de l'ordre de $10^{-4} \mathrm{M}_{\odot} /$ an ([118]).

$\| M \gtrsim 30 \mathrm{M}_{\odot}$; il faut noter que cette valeur limite dépend de la composition intiale de l'étoile et peut atteindre $80 \mathrm{M}_{\odot}$ environ pour des étoiles très pauvres en éléments plus lourds que $\mathrm{H}$ et $\mathrm{He}$ 


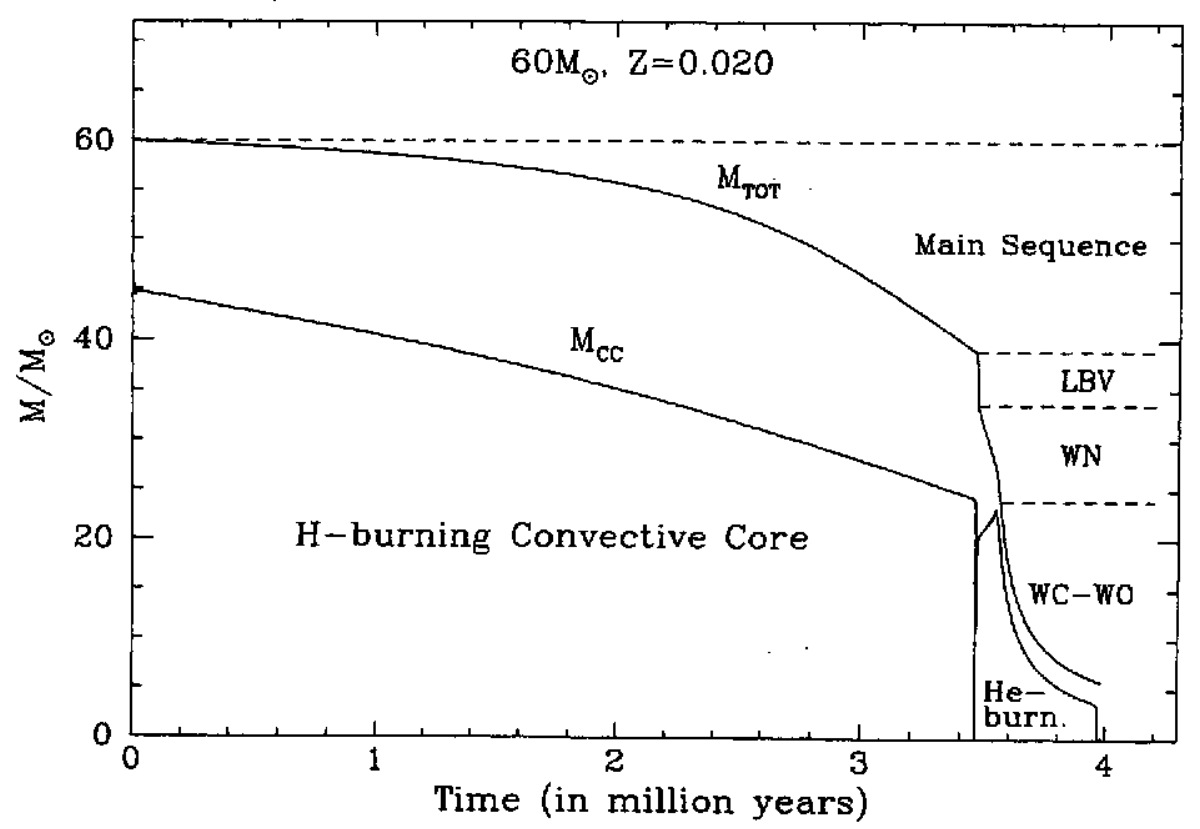

Figure 32. Evolution de la masse totale $\mathrm{M}_{\text {TOT }}$ et de la masse du coeur convectif où se produit la fusion de l'hydrogène d'une étoile de masse initiale de $60 \mathrm{M}_{\odot}$ et de métallicité solaire $(Z=0.02)$. Les différentes phases par lesquelles passe la surface de l'étoile sont mentionnées dans la partie droite de la figure. LBV se rapporte aux 'Variables Bleues Lumineuses' (Luminous Blue Variable), alors que WN et WC-WO indiquent des étoiles WR dont la surface est enrichie en $\mathrm{N}$ (provenant des cycles $\mathrm{CNO}$ ) et en C-O (produits de la combustion de $\mathrm{He}$ )

\section{Quelques Généralités sur la nucléosynthèse explosive}

\subsection{Un bref rappel des différents modes d'explosion stellaire}

Comme brièvement discuté à la Sect. 6.4.3, une explosion stellaire de type SNIa peut résulter d'une déflagration, détonation ou détonation 'retardée' (régime de déflagration se transformant en détonation) se développant au sein d'une naine blanche qui accrète de la matière d'une étoile compagne dans un système binaire. Par contre, les étoiles de masse $M \gtrsim 10 \mathrm{M}_{\odot}$ peuvent terminer leur évolution par un phénomène d'implosion induit par captures électroniques et par la photodésintégration d'une fraction substantielle de leur coeur de fer, cette implosion se transformant en supernova probablement sous l'effet de neutrinos qui diffusent des régions les plus centrales (étoile à neutrons). Ces neutrinos aident à la formation d'une onde de choc qui se propage vers la surface de l'étoile et est responsable de l'éjection explosive d'une quantité plus ou moins substantielle de matière. Cette explosion est de type SNII si la matière éjectée contient de l'hydrogène, ou de type SNIb/Ic si l'enveloppe riche en hydrogène est perdue par vents stellaires avant l'explosion. Outre ces explosions affectant toute la structure de l'étoile, certaines combustions hydrodynamiques ne concernent que la matière qui s'est accumulée à la surface d'une étoile à neutrons ou naine blanche et qui provient de l'autre étoile constituant un système binaire. Sous certaines conditions (en particulier la vitesse d'accrétion de matière), ces explosions peuvent donner naissance à des sursauts $\mathrm{x}$ à la surface d'étoiles à neutrons ou à des novae à la surface de naines blanches.

Ainsi que déjà souligné précédemment (Sect. 6.3), les problèmes hydrodynamiques posés par la modélisation de ces explosions sont d'une extrême complexité et sont loin d'être résolus aujourd'hui. Une partie des difficultés rencontrées vient de l'indispensable couplage entre mouvement du fluide stellaire et les réactions nucléaires importantes pour la 
production d'énergie et/ou la nucléosynthèse. Une approche souvent utilisée est de mener les calculs hydrodynamiques en utilisant un réseau restreint de réactions nucléaires essentielles à l'évaluation de l'énergétique. Les conditions physiques prédites par les modèles ainsi obtenus servent alors a posteriori de base à des calculs nucléosynthétiques faisant appel à des réseaux très étendus de réactions nucléaires. Ce découplage, s'il peut en général se justifier dans des approches hydrodynamiques à une dimension, s'avère être peu fiable dans le cadre de modèles multidimensionnels, la plus grande prudence s'imposant dans ce cas dans l'interprétation des prédictions d'abondances.

Rappelons enfin (Sect. 4.8) que les énergies d'intérêt astrophysique auxquelles se déroulent les réactions nucléaires sont typiquement plus élevées dans des situations explosives que non explosives, les sections efficaces de réaction étant conséquemment plus grandes. La situation est pourtant loin de s'en voir simplifiée pour autant, les flots nucléaires associés aux explosions impliquant une très grande variété de noyaux exotiques, nombre d'entre eux n'ayant jamais été observés en laboratoire.

\subsection{Les modes 'chauds' de combustion de l'hydrogène}

L'hydrogène peut brûler de manière explosive dans différents sites astrophysiques, comme les novae ou les sursauts $x$. Les modes de combustion 'chauds' correspondants impliquent une grande variété de noyaux instables. Ces modes ont des signatures nucléosynthétiques spécifiques et soulèvent de nombreuses difficultés en astrophysique et en physique nucléaire, tant sur le plan expérimental que théorique.

9.2.1. Les chaînes $p$-p chaudes Ce mode est susceptible de se développer dans les explosions de type nova ([87]). Parmi l'ensemble des réactions importantes, la photodésintégration ${ }^{8} \mathrm{~B}(\gamma, \mathrm{p}){ }^{7} \mathrm{Be}$ joue un rôle essentiel. Cette réaction entrave la transformation de ${ }^{7} \mathrm{Be}$ en ${ }^{4} \mathrm{He}$ (qui caractérise la chaîne $\mathrm{p}$-p froide) et pourrait ainsi être responsable d'une production de ${ }^{7} \mathrm{Li}$ (par désintégration de ${ }^{7} \mathrm{Be}$ ) dans les novae.

Les taux de différentes réactions du mode p-p chaud ont été étudiés théoriquement ou à l'aide de techniques expérimentales indirectes. En particulier, le taux de transformation de ${ }^{11} \mathrm{C}$ [résultant de ${ }^{7} \mathrm{Be}(\alpha, \gamma){ }^{11} \mathrm{C}$ ] par la réaction ${ }^{11} \mathrm{C}(\mathrm{p}, \gamma){ }^{12} \mathrm{~N}$ a été mesuré dans une expérience de 'break-up coulombien'. Le résultat diffère sensiblement des prédictions d'un modèle microscopique.

9.2.2. Les chaînes $C N O$ et $N e N a-M g A l$ chaudes Ces modes de combustion de $\mathrm{H}$ se développent à des températures suffisamment élevées pour que les noyaux instables produits se transforment plus rapidement par captures de protons, dont les vitesses augmentent fortement avec la température, que par désintégrations $\beta$, dont les vitesses sont essentiellement indépendantes de la température pour les noyaux légers impliqués en raison du manque de niveaux excités à assez basse énergie pour être suffisamment peuplés (Sect. 4.4.3). Cette situation est radicalement opposée à celle rencontrée dans les modes froids (Sect. 7.1). Plus spécifiquement, le cycle CNO froid se transforme en mode chaud lorsque ${ }^{13} \mathrm{~N}(\mathrm{p}, \gamma){ }^{14} \mathrm{O}$ devient plus rapide que la désintégration $\beta$ de ${ }^{13} \mathrm{~N}, \mathrm{c}$-à-d lorsque $T \gtrsim 10^{8}$ $\mathrm{K}$. Les modes $\mathrm{NeNa}$ et $\mathrm{MgAl}$ froids évoluent vers des modes chauds à des températures similaires, qui pourraient être rencontrées dans les novae.

De nombreux efforts théoriques et expérimentaux ont été consacrés à la détermination du taux de certaines réactions susceptibles de jouer un rôle important des les chaînes CNO et $\mathrm{NeNa}-\mathrm{MgAl}$ chaudes. En général, ces taux ne sont mesurés que par des techniques 
indirectes, mais quelques exceptions remarquables méritent d'être mentionnées. La première concerne ${ }^{13} \mathrm{~N}(\mathrm{p}, \gamma)^{14} \mathrm{O}$, dont la vitesse est aujourd'hui connue avec une précision suffisante pour les besoins astrophysiques [88]. Des expériences directes ont galement été menées sur ${ }^{22} \mathrm{Na}(\mathrm{p}, \gamma){ }^{23} \mathrm{Mg}$ et ${ }^{26} \mathrm{Al}{ }^{\mathrm{g}}(\mathrm{p}, \gamma){ }^{27} \mathrm{Si}$, canaux de destruction des radionucléides ${ }^{22} \mathrm{Na}$ et ${ }^{26} \mathrm{Alg}$ importants en astrophysique. Ces efforts expérimentaux ont permis de réduire fortement les incertitudes nucléaires affectant la production explosive de ${ }^{22} \mathrm{Na}$ et ${ }^{26} \mathrm{Al}$ dans les novae, comme on peut le constater en comparant la situation actuelle $[89,90]$ à celle prévalant dans les années 80 . Il faut cependant noter que d'importantes incertitudes affectent encore le taux de ${ }^{26} \mathrm{Al}{ }^{\mathrm{g}}(\mathrm{p}, \gamma)^{27} \mathrm{Si}$ aux basses températures caractéristiques des phases de l'évolution stellaire non explosive. Ces incertitudes n'ont cependant pas d'impact significatif sur les prédictions de l'abondance de ${ }^{26} \mathrm{Al}$ si la désintégration $\beta$ de ${ }^{26} \mathrm{Al}$ est le canal de destruction principal (voir [92] pour une illustration de cette situation dans le cas d'étoiles massives de type WolfRayet). D'autre part, si l'état isomérique ${ }^{26} \mathrm{Al}^{\mathrm{m}}$ possède une population thermique suffisante, sa contribution doit être prise en compte dans l'évaluation du taux effectif de destruction de ${ }^{26} \mathrm{Al}$ par capture de protons. Cette évaluation repose pour le moment essentiellement sur des calculs de type statistique [78]. Sa détermination expérimentale directe exigerait la production d'un faisceau de ${ }^{26} \mathrm{Al}^{\mathrm{m}}$, ce qui représente un défit technologique intéressant.

9.2.3. Les processus rp et $\alpha p$ Les modes $\mathrm{CNO}$ et $\mathrm{NeNa}-\mathrm{Mg}-\mathrm{Al}$ peuvent se transformer en processus appelés rp ou $\alpha$ p lorsque ${ }^{15} \mathrm{O}(\alpha, \gamma){ }^{19} \mathrm{Ne}$ ou ${ }^{14} \mathrm{O}(\alpha, \mathrm{p}){ }^{17} \mathrm{~F}$ deviennent plus rapides que les désintégrations $\beta$ correspondantes. La réaction ${ }^{18} \mathrm{Ne}(\alpha, \mathrm{p}){ }^{21} \mathrm{Na}$ pourrait également jouer ce rôle si sa vitesse devenait plus grande que celle de la désintégration $\beta$ de ${ }^{18} \mathrm{Ne}$.

Malgré les incertitudes encore importantes affectant les sections efficaces de ces réactions, il semble raisonnable d'admettre qu'elles peuvent se dérouler dans certaines supernovae de Type I ou dans les sursauts x. Dans ces conditions, le flot nucléaire, fait essentiellement de captures de protons et de désintégrations $\beta$, pourrait s'étendre de la région $\mathrm{C}-\mathrm{N}-\mathrm{O}$ jusqu'au pic du fer, ou même au-delà. Cette chaîne de transformations est appelée le processus rp ([126]). Elle pourrait se transformer en un processus $\alpha$ p à des températures suffisantes pour que des réactions $(\alpha, \mathrm{p})$ soient capables de court-circuiter des couples de réactions captures de protons + désintégrations $\beta$.

Une grande variété de noyaux instables déficients en neutrons, même proches de la ligne de drip protonique, pourraient être impliqués dans les processus rp et $\alpha$ p (certains parmi les plus légers d'entre eux intervenant déjà dans les modes CNO et $\mathrm{NeNa}-\mathrm{Mg}-\mathrm{Al}$ chauds). Une mesure directe d'une fraction importante des réactions potentiellement importantes est difficilement envisageable. A l'heure actuelle, et outre les expériences concernant ${ }^{13} \mathrm{~N}(\mathrm{p}, \gamma){ }^{14} \mathrm{O}$, seules ${ }^{18} \mathrm{~F}(\mathrm{p}, \alpha){ }^{15} \mathrm{O}$ et ${ }^{19} \mathrm{Ne}(\mathrm{p}, \gamma){ }^{20} \mathrm{Na}$ ont fait l'objet d'expériences dont les résultats peuvent être utilisables dans la modélisation astrophysique. Quelques résultats préliminaires existent aussi pour ${ }^{18} \mathrm{Ne}(\alpha, \mathrm{p})^{2 \mathbf{l}} \mathrm{Na}$. En fait, la plupart des informations relatives aux réactions rp et $\alpha$ p sont obtenues par des techniques expérimentales indirectes ou par la théorie.

Il est évident que beaucoup reste à faire pour placer les processus rp et $\alpha$ p sur une base nucléaire fiable. L'identification des réactions dont l'importance justifiererait un effort expérimental de grande envergure est loin d'être une tâche simple. Ainsi, il n'existe à ce jour aucune tentative sérieuse d'évaluation de l'impact d'incertudes nucléaires sur les propriétés observables d'objets aussi complexes que les sursauts x. Au contraire, une telle analyse se fonde sur la résolution de réseaux de réactions utilisant des conditions physiques simplifiées à l'extrême ([126]). Cette procédure peut conduire à une évaluation erronée de la véritable importance pour les processus rp et $\alpha$ p de certains nucléides ou de certaines réactions. 


\subsection{La combustion explosive de He jusqu'à $S i$}

La combustion explosive des éléments $\mathrm{He}, \mathrm{C}, \mathrm{Ne}, \mathrm{O}$ et $\mathrm{Si}$ peut se produire dans différentes situations, comme l'explosion d'étoiles massives isolées, l'accrétion ou même la coalescence de naines blanches dans les systèmes binaires, ainsi que l'accrétion de matière à la surface d'étoiles à neutrons. Comme dans le cas de la combustion explosive de $\mathrm{H}$, ces modes de combustion chaude impliquent une variété de réactions sur des cibles instables qui ne jouent aucun rôle significatif dans les modes froids correspondants.

Ces différents modes de combustion explosive ont été étudiés dans le cadre de modèles paramétriques ou plus détaillés $([11,115,127])$.

\section{La nucléosynthèse associée à l'explosion d'étoiles massives}

Ainsi que déjà mentionné à la Sect. 6.4.1, l'explosion d'une étoile massive en supernova (SNII ou SNIb/Ic) est associée à la propagation d'une onde de choc au travers des diverses couches de l'étoile schématisée à la Fig. 20. L'onde de choc comprime ces couches et les échauffe avant de provoquer leur éjection dans le milieu interstellaire, et donc leur refroidissement. Ce processus d'échauffement et de refroidissement s'accompagne de la combustion explosive de He jusqu'au Si. En raison de la brièveté de cet épisode nucléosynthétique, les abondances pré-explosives des nucléides les plus abondants sont en général relativement peu affectées, contrairement aux nucléides plus rares. La composition de l'éjecta de SNII de différentes masses et de composition initiale (métallicité) comparable à celle du système solaire est représentée à la Fig. 33 sous l'hypothèse d'un mélange parfait des couches expulsées. Il apparaît que la signature de la combustion de $\mathrm{C}$ (produisant essentiellement des noyaux dans le domaine de masse approximatif $20 \lesssim A \lesssim 40$ ) augmente avec la masse stellaire, la situation inverse se manifestant pour la combustion de $\mathrm{Si}$. responsable des noyaux du pic du fer $(45 \lesssim A \lesssim 65)$.

Il faut noter que les prédictions présentées à la Fig. 33 souffrent de très nombreuses incertitudes. Certaines sont d'origine nucléaire. En particulier, les incertitudes subsistant dans la vitesse de la réaction ${ }^{12} \mathrm{C}(\alpha, \gamma){ }^{16} \mathrm{O}$ ont un impact direct sur les abondances relatives des produits de la combustion de $\mathrm{C}$ et de $\mathrm{O}$. De nombreuses incertitudes d'origine astrophysique affectent également les prédictions. Ainsi, l'abondance des noyaux du pic du fer dans l'éjecta dépend de façon sensible de la position précise de l'interface entre matière éjectée et résidu de la supernova (étoile à neutrons ou trou noir). La localisation de cette interface ne peut aujourd'hui être prédite de façon fiable à partir de principes premiers et est en réalité un paramètre libre choisi afin de reproduire au mieux certaines observations. Parmi celles-ci, il convient de citer la courbe de lumière (variation de l'éclat en fonction du temps) de la supernova SN1987A du Grand Nuage de Magellan, qui semble ne pouvoir s'interpréter sur des échelles de temps de l'ordre de quelques centaines de jours après l'explosion que si environ $0.07 \mathrm{M}_{\odot}$ de ${ }^{56} \mathrm{Ni}$ se désintègre suivant ${ }^{56} \mathrm{Ni}\left(t_{1 / 2} \approx 6\right.$ jours $) \rightarrow{ }^{56} \mathrm{Co}\left(t_{1 / 2} \approx 77\right.$ jours) $\rightarrow{ }^{56} \mathrm{Fe}$ en déposant dans l'éjecta l'énergie correspondant à cette transformation. A plus long terme, l'énergie libérée par la désintégration de ${ }^{57} \mathrm{Co}$, puis de ${ }^{44} \mathrm{Ti}$, peut également contribuer à la courbe de lumière. La présence de ${ }^{56} \mathrm{Co}$ et de ${ }^{57} \mathrm{Co}$ dans l'éjecta de SN1987A est également attestée de façon directe par l'observation de raies $\gamma$ nucléaires associées à la désintégration de ces radionucléides. La réalité de la nucléosynthèse explosive, seule capable de produire ${ }^{44} \mathrm{Ti},{ }^{56} \mathrm{Ni},{ }^{56} \mathrm{Co}$ et ${ }^{57} \mathrm{Co}$, ne peut donc plus être mise en doute !

$\mathrm{Au}$ contraire, les modèles à symétrie sphérique de supernovae adoptés pour mener les calculs de nucléosynthèse explosive semblent présenter de plus en plus de déficiences tant sur le plan observationnel que théorique. Les observations d'éjecta contredisant la 


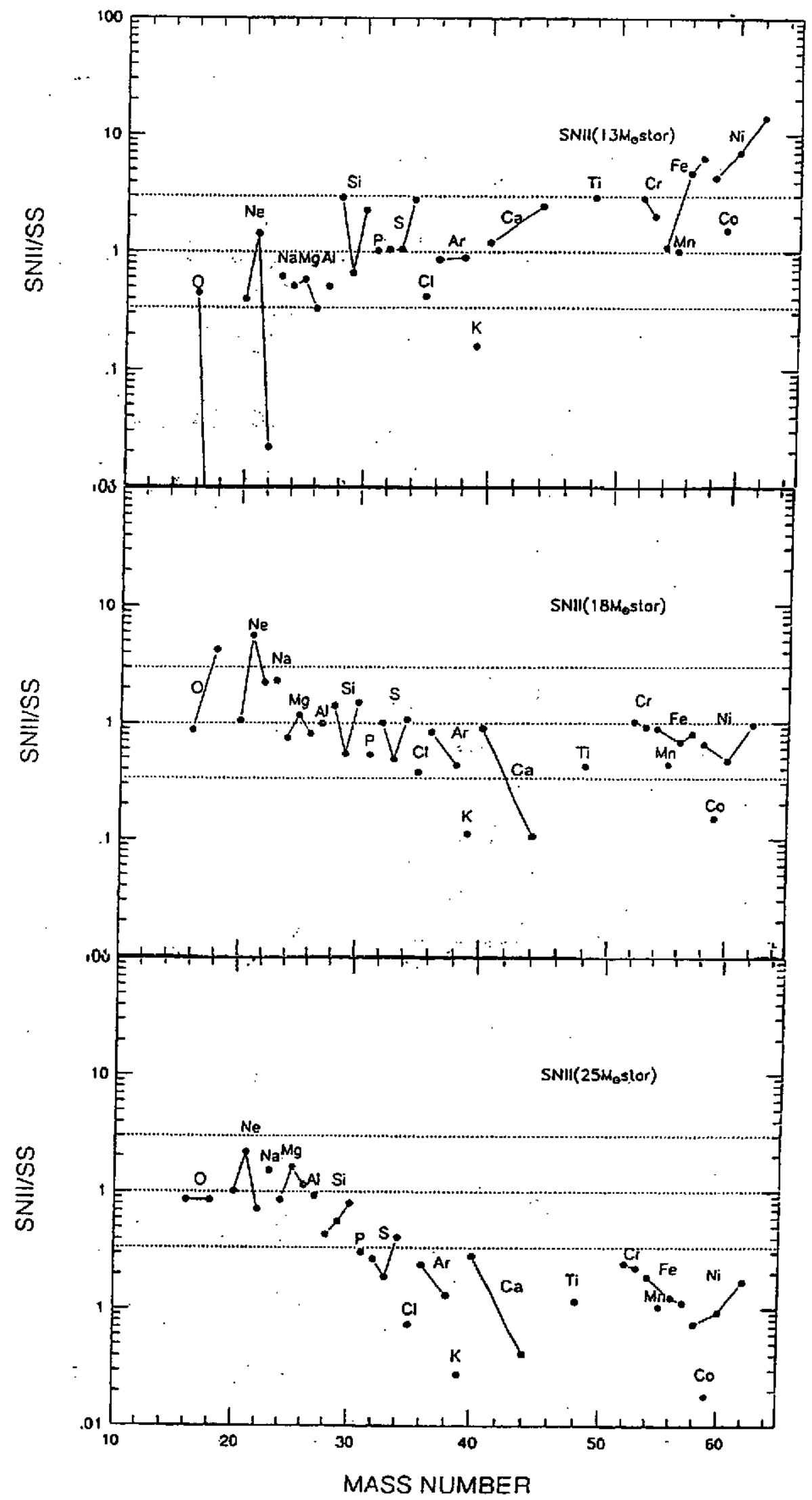

Figure 33. Composition de l'éjecta de SNII de diverses masses comparée à celle du système solaire (SS). La matière éjectée est supposée chimiquement homogène 

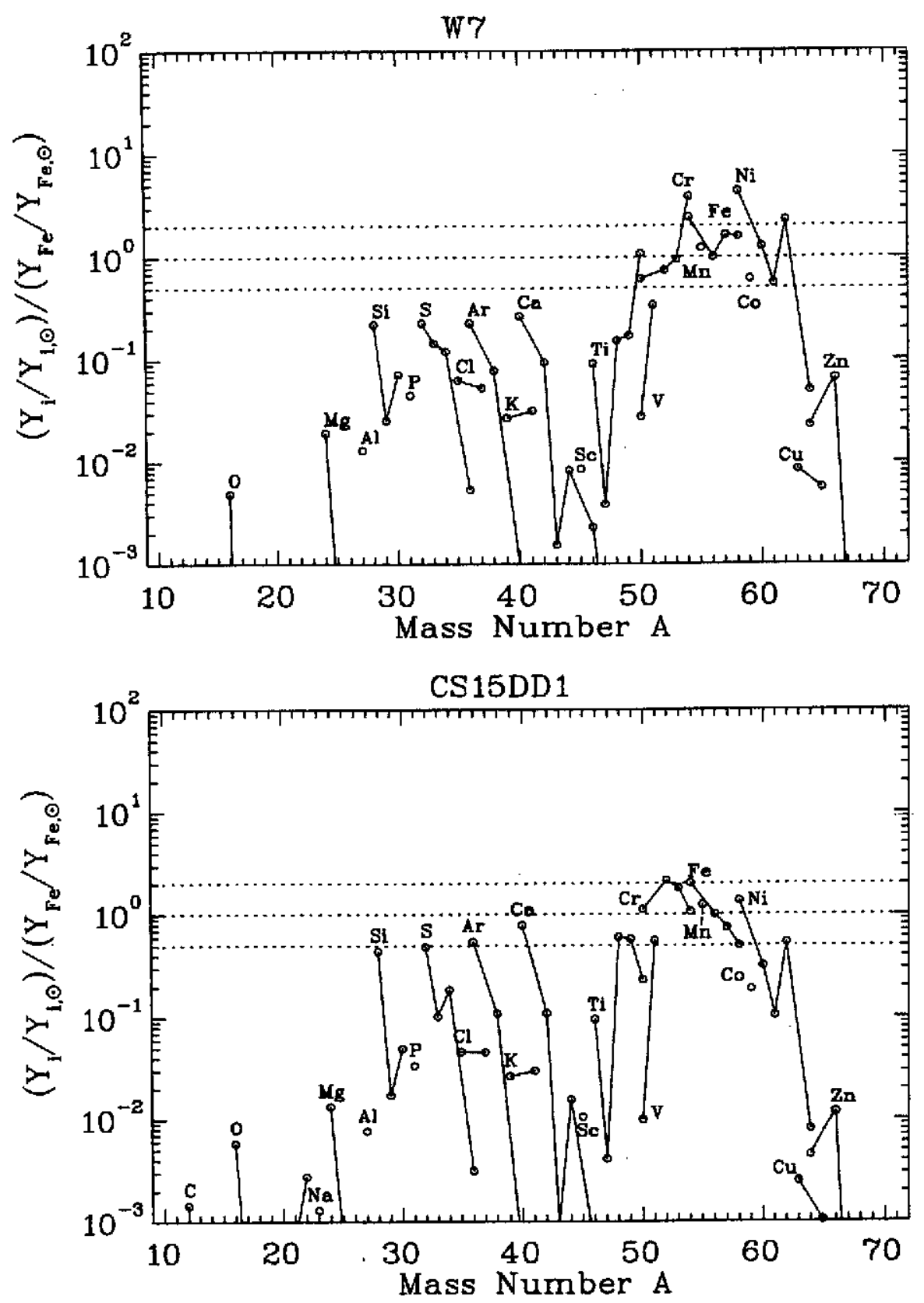

Figure 34. Composition de l'éjecta de SNIa résultant d'une déflagration 'rapide' (vitesse de flamme pouvant atteindre plus de $10 \%$ de la vitesse du son; figure supérieure) et d'une déflagration retardée (figure inférieure) d'une étoile naine blanche de masse égale à la limite de Chandrasekhar. Les abondances molaires $Y_{i}$ rapportées aux valeurs solaires sont normalisées au rapport d'abondances de Fe. Tous les modèles sont à symétrie sphérique (figures tirées de [128])

symétrie sphérique et démontrant l'existence d'importantes inhomogénéités de composition s'accumulent, tandis que l'observation de raies $\gamma$ nucléaires dans SN1987A beaucoup plus tôt après l'explosion que prévu par les modèles démontre le développement dans la matière éjectée de grandes variations structurelles à diverses échelles dues à des instabilités de nature hydrodynamique. Ces caractéristiques ne peuvent évidemment être prédites par des modèles à symétrie sphérique. 


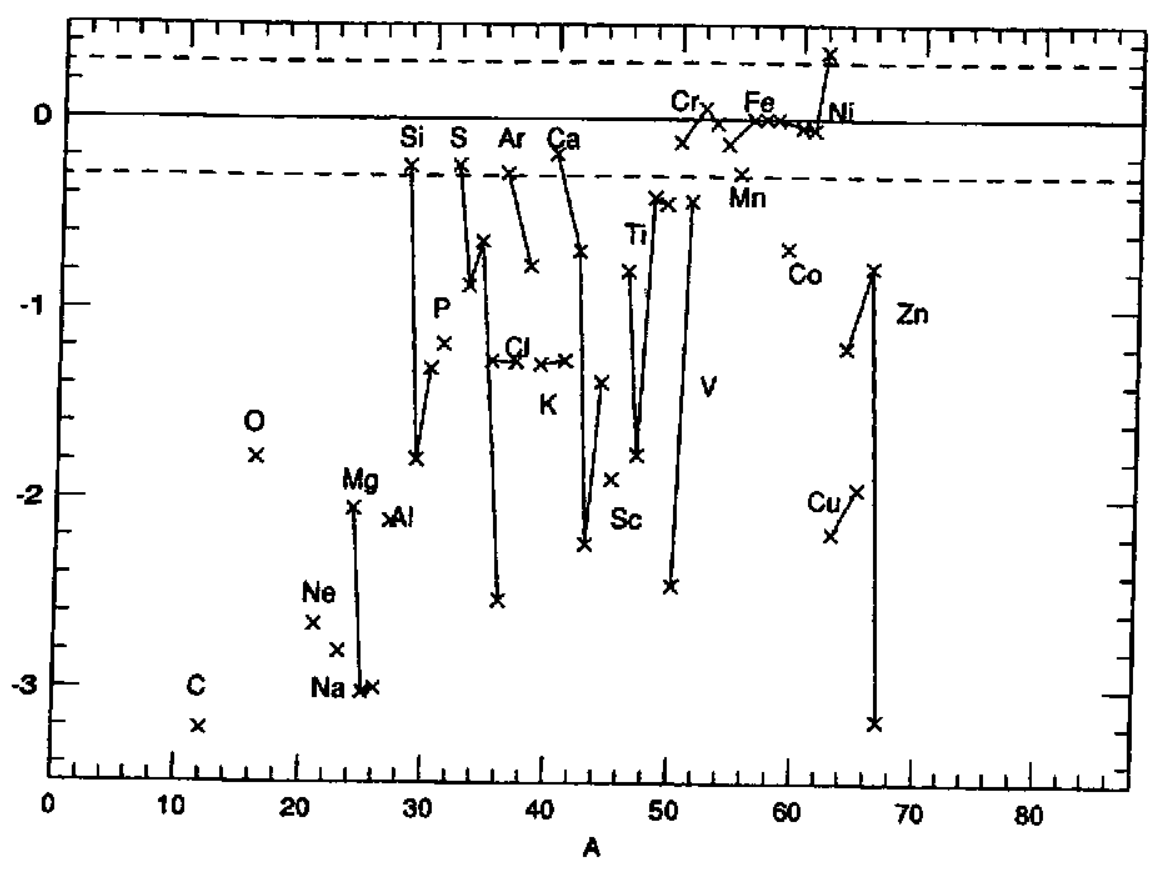

Figure 35. Identique à la Fig. 34, mais pour l'explosion d'une étoile naine blanche de masse $M=1.05 \mathrm{M}_{\odot}$ (inférieure à la limite de Chandrasekhar) résultant d'une détonation non centrale du carbone [129]

\subsection{La nucléosynthèse explosive accompagnant les détonations et déflagrations}

Ainsi que souligné précédemment (Sect. 6.3), les combustions hydrodynamiques, et tout particulièrement les régimes de déflagration ou de détonation retardée, soulèvent d'énormes problèmes de modélisation. Toute prédiction de la nucléosynthèse concomitante doit donc être considérée avec beaucoup de réserve. La Fig. 34 présente quelques résultats obtenus dans le cadre d'un modèle de déflagration et de déflagration retardée dans des naines blanches dont la masse est égale à celle de Chandrasekhar. 9 Ces prédictions sont très sensibles au choix des valeurs d'un certain nombre de paramètres libres, et en particulier des vitesses de propagation de la flamme et de la densité à laquelle s'opère la transition de la déflagration à la détonation dans le modèle de déflagration retardée. Une caractéristique générale des modèles de ce type est l'importante production de noyaux du pic du fer, ainsi que de quelques nucléides de masse inférieure, dont la présence dans l'éjecta est requise par les observations spectroscopiques des SNIa. L'absence de production en quantité significative de noyaux plus légers que le pic du fer dans les régimes de détonation pure de naines blanches de masse proche de celle de Chandrasekhar a conduit à l'abandon de tels modèles. La situation pourrait être différente pour des naines blanches de masse inférieure à celle de Chandrasekhar, qui pourraient notamment être le siège d'une détonation non centrale de l'hélium ou du carbone. Un exemple de composition de l'éjecta résultant d'une telle détonation est représenté à la Fig. 35. Il est généralement considéré que les modèles d'explosion d'une telle naine blanche sont difficilement compatibles avec les caractéristiques observées de SNIa typiques.

Des modèles hydrodynamiques multidimensionnels commencent à voir le jour et pourront certainement aider à résoudre certains au moins des problèmes conceptuels majeurs posés par les simulations à une dimension des combustions de type détonation ou déflagration. Beaucoup reste cependant à faire en ce domaine particulièrement complexe.

I La masse de Chandrasekhar est la limite supérieure de la masse d'une étoile naine blanche susceptible d'être à l'équilibre mécanique grâce à la pression exercée par un gaz d'électrons dégénérés. Cette limite est
approximativement de $1.4 \mathrm{M}_{\odot}$ 


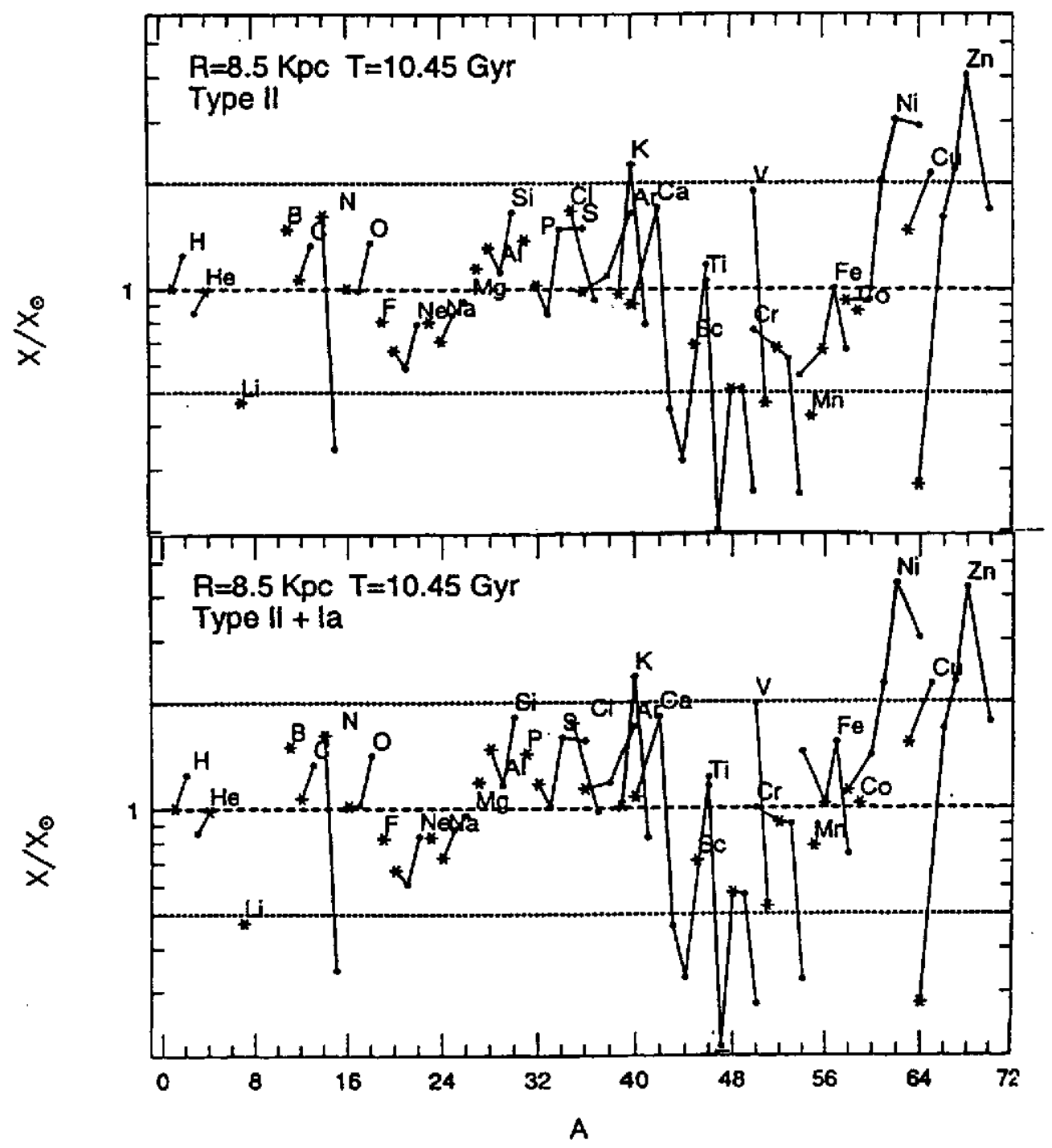

Figure 36. Composition de l'hydrogène au zinc prédite par un modèle d'évolution chimique de la Galaxie de la matière à partir de laquelle s'est formé le système solaire. Cet événement est supposé s'être produit $10.45 \times 10^{9}$ après la formation de la Galaxie (c-à-d voici environ $4.55 \times 10^{9}$ ans) et à une distance de $8.5 \mathrm{kpc}$ du centre galactique. Les abondances calculées sont normalisées à celles mesurées dans le système solaire. La figure supérieure (inférieure) est obtenue en négligeant (incluant) la contribution des SNII, tandis que l'apport complémentaire des SNIa est pris en compte dans la figure inférieure (tiré de [130])

\subsection{Les supernovae et l'évolution chimique de la Galaxie}

L'apport au MIS de nucléides fraîchement synthétisés par les supernovae de diverses masses et compositions initiales est l'un des agents les plus importants de l'évolution chimique des galaxies en général, et de la Voie Lactée en particulier. La Fig. 36 présente les résultats d'un modèle simple d'évolution chimique (Sect. 3) prenant en compte une composition initiale prédite par un modèle de Big Bang standard (Sect. 3), la contribution des vents d'étoiles de masse inférieure à $8 \mathrm{M}_{\odot}$ passant par la phase nébuleuse planétaire (Sect. 6.4.2), ainsi que les explosions de type SNIa et SNII. Par contre, la contribution des vents d'étoiles massives de type Wolf-Rayet (Sect. 8 et Fig. 9) est négligée, de même que la production spallative du $\mathrm{Li}$, Be et $B$ par le rayonnement cosmique galactique, la synthèse de ces noyaux étant assignée au processus $\nu$ (Sect. 4.4.2). Les abondances représentées (entre $\mathrm{H}$ et $\mathrm{Zn}$; pour les éléments plus lourds, voir le cours de S. Goriely) se rapportent à la composition de la matière galactique 


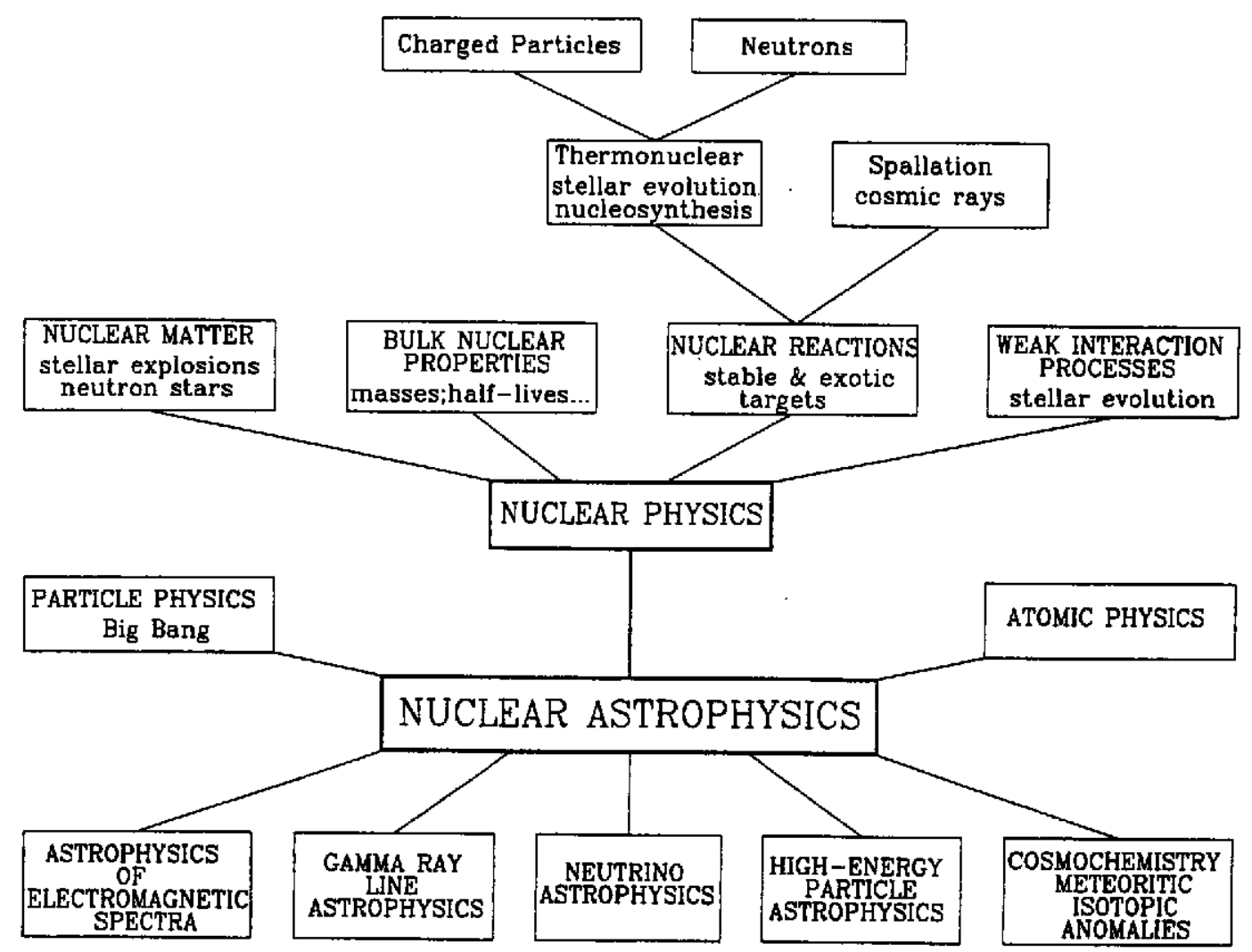

Figure 37. Représentation très schématique des relations possibles entre astrophysique nucléaire et d'autres disciplines majeures de la physique ou d'autres chapitres importants de l'astrophysique (tiré de [13])

à partir de laquelle le système solaire s'est formé. Un ensemble de prédictions ne s'écartent guère des observations, la situation la moins satisfaisante étant rencontrée pour les noyaux du pic du fer $(A \gtrsim 45)$. Il est dès lors difficile d'évaluer de façon fiable la contribution relative des SNIa et des SNII aux abondances solaires de ces nucléides. De $1 / 3$ à $2 / 3$ du fer solaire pourraient venir des SNIa [130].

Plus généralement, les prédictions de tous les modèles d'évolution chimique des galaxies construits à ce jour sont entachées de multiples et graves incertitudes de nature astrophysique et nucléaire relatives à la nucléosynthèse dans des étoiles individuelles, ainsi que d'incertitudes dans les autres ingrédients de base des modèles d'évolution galactique (Sect. 3). En ce domaine, le chemin restant à parcourir est particulièrement long!

\section{Résumé}

L'astrophysique nucléaire est sans nul doute une branche largement interdisciplinaire de la science. Comme schématisé à la Fig. 37, elle est intimement connectée à une très grande variété d'autres domaines de recherche et constitue une véritable boîte de Pandore de questions scientifiques. Ce cours s'est limité à une brève présentation des relations privilégiées qui se sont établies entre l'astrophysique et la physique nucléaire.

Nous avons tenté de montrer que la physique nucléaire et l'astrophysique apportent une contribution importante à la compréhension d'une ensemble rapidement croissant d'observations relatives à l'Univers et à ses constituants les plus divers, allant de grains 
sub-millimétriques dans les météorites à des galaxies extrêmement lointaines. Nous avons également illustré le fait que l'astrophysique pousse très souvent la physique nucléaire dans ses 'derniers retranchements' et à explorer des 'terres inconnues' situées bien loin de la vallée de stabilité nucléaire.

Des progrès considérables ont été réalisés dans cette exploration et la fin de ce siècle a été particulièrement riche en découvertes et en progrès de toutes sortes. Malgré cela, bien du travail reste à faire, comme illustré à diverses occasions dans ce cours.

A l'aube d'un nouveau millénaire, il est difficile de prévoir l'évolution de l'astrophysique nucléaire à moyen ou à long terme. La seule certitude est qu'une meilleure compréhension mutuelle entre physiciens nucléaires et astrophysiciens ne pourrait manquer d'être la source de nouvelles et exaltantes aventures scientifiques.

\section{References}

[1] Russell H N 1919 Pub. Astron. Soc. Pac. 31 205-11

[2] Perrin J 1920 Scientia 30 355-70

[3] Hoyle F 1946 Mon. Not. Roy. Astron. Soc. 106 343-83

[4] Merrill P W 1952 Astrophys. J. 116 21-6

[5] Burbidge E M et al. 1957 Rev. Mod. Phys. 29 547-650

[6] Öpik E J 1951 Proc. Roy. Irish Acad. A54 49-77

[7] Salpeter E E 1952 Astrophys. J. 115 326-8

[8] Hoyle F 1954 Astrophys. J. Suppl. 1 121-46

[9] Clayton D D 1968 Principles of Stellar Evolution and Nucleosynthesis (New York: MacGraw-Hill)

[10] Rolfs C E and Rodney W S 1988 Cauldrons in the Cosmos (Chicago: Univ. Chicago Press)

[11] Arnett W D 1996 Supernovae and Nucleosynthesis (Princeton: Princeton Univ. Press)

[12] Pagel B E J 1997 Nucleosynthesis and Chemical Evolution of Galaxies (Cambridge: Cambridge Univ. Press)

[13] Arnould M and Takahashi T 1999 Rep. Prog. Phys. 62395 - 464

[14] Léna P 1988 Observational Astrophysics (Berlin: Springer-Verlag)

[15] Böhm-Vitense E 1989 Introduction to Stellar Astrophysics Vol. 1 (Cambridge: Cambridge Univ. Press)

[16] Cox J P and Giuli R T 1968 Principles of Stellar Structure (New York: Gordon and Breach)

[17] Sandage A 1988 Calibration of Stellar Ages Philip A G D (ed) (Schenectady: L. Davis Press) pp. 43-58

[18] Reeves H 1994 Rev. Mod. Phys. 66 193-216

[19] McWilliam A 1997 Ann. Rev. Astron. Astrophys. 35 503-56

[20] Kahane C. 1995 Nuclei in the Cosmos (AIP Conf. Proc. 32\%) vol III. ed M Busso R Gallino and C M Raiteri (New York: AIP) pp. 19-30

[21] Savage B D and Sembach K R 1996 Ann. Rev. Astron. Astrophys. 34 279-329

[22] Lu L et al. 1996 Astrophys. J. Suppl. 107 475-519

[23] Tayler R J 1986 Q. $\pi$. R. astr. Soc. 27 367-82

[24] Fowler W A and Meisl C C 1986 Cosmogonical Processes Arnett W D et al. (eds) 1986 (Utrecht: VNU Sci. Press) pp. 83-100

[25] Arnould M and Takahashi K 1990 New Windows to the Universe Sanchez F and Vazquez M (eds) (Cambridge: Cambridge Univ. Press) pp. 355-74

[26] Smith V V 1989 Cosmic Abundances of Matter [AIP Conf. Proc. 183] Waddington C J (ed) (New York: Amer. Inst. Phys.) pp. 200-23

[27] Gehrz R D et al. 1998 Pub. Astron. Soc. Pac. 110 3-26

[28] Filippenko A V 1997 Ann. Rev. Astron. Astrophys. 35 309-55

[29] Vauclair S and Vauclair G 1982 Ann. Rev. Astron. Astrophys. 20 37-60

[30] Anders E and Grevesse N 1989 Geochim. Cosmochim. Acta 53 197-214

[31] Grevesse N and Sauval A J 1998 Space Sci. Rev 85161 - 174

[32] Harper C L Jr 1993 Nuclei in the Cosmos vol II, ed F. Käppeler and K Wisshak (Bristol: IOP)l pp. $113-26$

[33] Bernatowicz T J and Zinner E (eds) 1997 Astrophysical Implications of the Laboratory Study of Presolar Materials [AIP Conf. Proc. 402] (New York: Amer. Inst. Phys.)

[34] Podosek F A and Nichols R H Jr 1997 Astrophysical Implications of the Laboratory Study of Presolar 
Materials loc. cit. [33] pp. 617-47

[35] Meyer J-P 1993 Origin and Evolution of the Elements ed N Prantzos, E Vangioni-Flam and M Cassé (Cambridge: Cambridge University Press) pp. 26-62

[36] Meyer J -P, Drury O'C and Ellison D C 1997 Astrophys. J. 487 182-96

[37] Ellison D C, Drury O'C and Meyer J -P 1997 Astrophys. J. 487 197-217

[38] Shore S N 1997 Nuclei in the Cosmos IV, ed J. Görres et al, Nucl. Phys. A621 pp. 535c-47c

[39] Scalo J M 1986 Fund. Cosmic Phys. 11 1-278

[40] Coc A, Porquet M G and Nowacki F 2000 Phys. Rev. C61 015801

[41] Vanhorenbeeck J et al. 2000 Phys. Rev. 0000000

[42] Lesko K T et al. 1991 Phys. Rev. C44 2850-64

[43] Hillebrandt 1991 High-Pressure Equations of State: Theory and Applications Eliezer S and Ricci R A (eds) ["Enrico Fermi" Course 113] (Amsterdam: North-Holland Pub.) pp. 399-437

[44] Pethick C J and Ravenhall D G 1995 Ann. Rev. Nucl. Part. Sci. 45 429-84

[45] Grotz K and Klapdor H V 1990 The Weak Interaction in Nuclear, Particle and Astrophysics (Bristol: Adam Hilger)

[46] Bravo E et al. 1983 Astron. Astrophys. 124 39-42

[47] Hashimoto $M$ et al. 1986 Astrophys. J. 307 687-93

[48] Hashimoto M, Iwamoto K and Nomoto K 1993 Astrophys. J. 414 L105-8

[49] Bethe H A 1990 Rev. Mod. Phys. 62 801-66

[50] Mayle R W 1990 Supernovae Petschek A G (ed) (New York: Springer) pp. 267-89

[51] Bruenn S W and Haxton W C 1991 Astrophys. J. 376 678-700

[52] Janka H-Th 1993 Frontier Objects in Astrophysics and Particle Physics, Giovannelli F and Mannocchi G (eds) (Bologna: Società Italiana di Fisica) pp. 345-74

[53] Woosley S E et al. 1990 Astrophys. J. 356 272-301

[54] Nadyozhin D K 1991 Nuclear Astrophysics 6 Hillebrandt W and Müller E (eds) [MPA-report P5]. (Garching: Max-Planck-Institut f. Astrophysik) pp. 118-22 [unpublished]

[55] Konopinski E J 1966 The Theory of Beta Radioactivity (Oxford: Clarendon Press)

[56] Tubbs D L and Schramm D N 1975 Astrophys. J. 201 467-88

[57] Bruenn S W 1985 Astrophys. J. Suppl. 58 771-841

[58] Raffelt G, Seckel D and Sigl G 1996 Phys. Rev. D54 2784-92

[59] Barrows A and Sawyer R F 1998 Phys. Rev. C58 554-71

[60] Yamada S 1998 Nuclear Astrophysics vol 9, ed W Hillebrandt and E Müller (Garching: Max-PlanckInstitut f. Astrophysik), pp. 115-8

[61] Daudel R et al. 1947 C. R. Acad. Sci. 224 1427-9

[62] Jung $M$ et al. 1992 Phys. Rev. Lett. $692164-67$

[63] Bosch F et al. 1996 Phys. Rev. Lett. 775190 - 93

[64] Takahashi K and Yokoi K 1983 Nucl. Phys. A404 578-98

[65] Takahashi K and Yokoi K 1987 Atm. Nucl. Data Tables 36 375-409

[66] Simpson J A and Garcia-Munoz M 1988 Space Sci. Rev. 46 205-24

[67] Cassé M 1973 Astrophys. J. 180 623-9

[68] Wiedenbeck M E et al. 1999 Astrophys. J. 523 L61-4

[69] Wietfeldt F E et al. 1999 Phys. Rev. C59 528

[70] Mochizuki $\mathrm{Y}$ et al. $1999{ }^{A)} 346831-842$

[71] Sumiyoshi K et al. 1998 Astron. Astrophys. 334 159-68

[72] Perrone F A and Clayton D D 1971 Astrophys. Space Sci. 11 451-62

[73] Rolfs C 1993 Origin and Evolution of the Elements ed N Prantzos, E Vangioni-Flam et M Cassé (Cambridge: Cambridge University Press) pp. 66-76 [74] Angulo C et al. 1992 Nuclei in the Cosmos vol II, ed F Käppeler et K Wisshak (Bristol: IOP) pp.

[75] Angulo C et al. 1993 Z. Phys. A345 231-42

[76] Dzitko $\mathrm{H}$ et al. 1993 Origin and Evolution of the Elements ed N Prantzos, E Vangioni-Flam et M Cassé (Cambridge: Cambridge University Press) pp. 388-91

[77] Görres J et al. (eds) 1997 Nuclei in the cosmos IV [Nucl. Phys. A621]

[78] Angulo C, Arnould M, Rayet M and the NACRE Collaboration 1999 Nucl. Phys. A656 3-183

[79] Weaver T and Woosley S E 1993 Phys. Rep. 227 65-96

[80] Arnett E and Thielemann F-K 1985 Astrophys. J. 295589

[81] Chiosi C and Maeder A 1986 Ann. Rev. Astron. Astrophys. 24 329-75

[82] Nomoto K and Hashimoto M 1988 Phys. Rep. 16313

[83] Jorissen A and Arnould M 1989 Astron. Astrophys. 221161 
[84] Arnould M and Howard W M 1976 Nucl. Phys. A274 295-332

[85] Descouvemont P 1989 Nucl. Phys. A504 193-204

[86] Woosley S E, Arnett W D and Clayton D D 1973 Astrophys. J. Suppl. 26 231-312

[87] Arnould M and Nørgaard H 1975 Astron. Astrophys. 42 55-70

[88] Arnould M, Paulus G and Jorissen A 1992 Astron. Astrophys. 254 L9-12

[89] Coc A et al. 1995 Astron. Astrophys. 299 479-92

[90] Coc A and Porquet M-G 1998 Tours Symposium on Nuclear Physics III, eds. M Arnould et al., AIP Conf. Series, vol. 425, pp. 457-64

[91] Arnould M 1980 Explosive Nucleosynthesis, Cahier n8, ed. M. Demeur (Physique Nucléaire Théorique, Université Libre de Bruxelles)

[92] Meynet G et al. 1997 Astron. Astrophys. 320 460-8

[93] Caughlan G R and Fowler W A 1988 Atm. Nucl. Data Tables 40283

[94] Arnould M, Goriely S and Jorissen A 1999 Astron. Astrophys. 347 572-582

[95] Sarkar S 1996 Rep. Prog. Phys. 59, 1493 - 1610 (révision: astro-ph/9602260, 1996)

[96] Sarkar S 1999 astro-ph/9903183

[97] Olive K A, Steigman G and Walker T P 1999 Phys. Rep 333 389-407

[98] Tytler D, O'Meara J M, Suzuki N and Lubin D 2000 astro-ph/0001318

[99] Vangioni-Flam E, Coc A, Cassé M 2000 Astron. Astrophys. 360 15-23

[100] Fields B and Olive K A 1998 Astrophys. J. 506177

[101] Izotov Y I and Thuan T X 1998 Astrophys. J. 5001888

[102] Webb J K et al. 1997 Nature 383, 250

[103] Molaro P 1999 in LiBeB, Cosmic Rays, and Related $X$ and Gamma-rays, eds. R Ramaty, E VangioniFlam, M Cassé and K Olive, ASP Conf. Series, vol 171, p. 6

[104] Ryan S G, Norris I and Beers T C 1999 astro-ph/9903059

[105] Smith M S, Kawano L H and Malaney R A 1993 Astrophys. J. Suppl. 85 219-47

[106] Orito $\mathrm{M}$ et al. 1997 Astrophys. J. 488 515-23

[107] Malaney R A and Mathews G J 1993 Phys. Rep. 229 145-219

[108] Forestini M 1999 Principes fondamentaux de structure stellaire (France: Gordon and Breach Science Pub.)

[109] Arnett D and Bazán G 1997 Science 276 1359-62

[110] Bazán G and Arnett D 1998 Astrophys. J. 496 316-32

[111] Pinsonneault M 1997 Ann. Rev. Astron. Astrophys. 35 557-605

112] Dupree A K 1986 Ann. Rev. Astron. Astrophys. 24 377-420

[113] Hashimoto M 1995 Prog. Theor. Phys. 94 663-736

[114] Chevalier R A 1997 Science 276 1374-8

[115] Nomoto K, Iwamoto K and Kishimoto N 1997 Science 276 1378-82

[116] Srinivasan G 1997 Stellar Remnants ed G Meynet and D Schaerer (Berlin: Springer) pp. 97-235

[117] Novikov I 1997 Stellar Remnants ed G Meynet and D Schaerer (Berlin: Springer) pp. 237-334

[118] Arnould M, Meynet G and Paulus G 1997 Astrophysical Implications of the Laboratory Study of Presolar Materials loc. cit. [33] pp. 179-202

[119] Woosley S E, Langer N and Weaver T A 1995 Astrophys. J. 448 315-38

[120] Janka H-Th and Müller E 1996 Astron. Astrophys. 306 167-98

[121] Khokhlov A and Höflich P 2000 1st KIAS Astrophysics Workshop, Seoul/Corea ed. I. Yi (IAP Publishing) in press; astro-ph/0011023

[122] Lattanzio J C and Boothroyd A I 1997 Astrophysical Implications of the Laboratory Study of Presolar Materials loc. cit. [33] pp. 85-114

[123] Nussbaumer H and Orr A (eds) 1994 Interacting Binaries [Saas-Fee Advanced Course 22] (Berlin: Springer-Verlag)

[124] Hillebrandt W and Niemeyer J C 2000 Ann. Rev. Astron. Astrophys. 38 191-230

[125] Costa V, Rayet M, Zappalà R A and Arnould M 2000 Astron. Astrophys. ??? ???

[126] Schatz H et al. 1998 Phys. Rep. 294 167-263

[127] Woosley S E and Weaver T A 1995 Astrophys. J. Suppl. $101181-235$

[128] Iwamoto K et al. 1999 Astrophys. J. Suppl. $125439-462$

[129] Nomoto K, Yamaoka H and Shigeyama T 1996 Supernovae and Supernova Remnants [IAU Coll. 45] ed R McCray and Z. Wang (Cambridge: Cambridge Univ. Press) pp. 49 - 68

[130] Timmes F X, Woosley S E and Weaver T A 1995 Astrophys. J. Suppl. 88617 - 658 